\title{
A taxonomic framework for typhlopid snakes from the Caribbean and other regions (Reptilia, Squamata)
}

\author{
S. Blair Hedges ${ }^{1, *}$, Angela B. Marion ${ }^{1}$, Kelly M. Lipp ${ }^{1,2}$, Julie Marin ${ }^{3,4}$, and Nicolas Vidal ${ }^{3}$ \\ 'Department of Biology, Pennsylvania State University, University Park, PA 16802-5301, USA. \\ ${ }^{2}$ Current address: School of Dentistry, University of North Carolina, Chapel Hill, NC 27599-7450, USA. \\ ${ }^{3}$ Département Systématique et Evolution, UMR 7138, C.P. 26, Muséum National d'Histoire Naturelle, 57 rue Cuvier, F-75231 Paris cedex 05, \\ France. \\ ${ }^{4}$ Current address: Department of Biology, Pennsylvania State University, University Park, PA 16802-5301 USA. \\ *Corresponding author (sbh1@psu.edu)
}

Article registration: http://zoobank.org/urn:Isid:zoobank.org:pub:47191405-862B-4FB6-8A28-29AB7E25FBDD

Edited by: Robert W. Henderson. Date of publication: 17 January 2014.

Citation: Hedges SB, Marion AB, Lipp KM, Marin J, Vidal N. 2014. A taxonomic framework for typhlopid snakes from the Caribbean and other regions (Reptilia, Squamata). Caribbean Herpetology 49:1-61.

\begin{abstract}
The evolutionary history and taxonomy of worm-like snakes (scolecophidians) continues to be refined as new molecular data are gathered and analyzed. Here we present additional evidence on the phylogeny of these snakes, from morphological data and 489 new DNA sequences, and propose a new taxonomic framework for the family Typhlopidae. Of 257 named species of typhlopid snakes, 92 are now placed in molecular phylogenies along with 60 additional species yet to be described. Afrotyphlopinae subfam. nov. is distributed almost exclusively in sub-Saharan Africa and contains three genera: Afrotyphlops, Letheobia, and Rhinotyphlops. Asiatyphlopinae subfam. nov. is distributed in Asia, Australasia, and islands of the western and southern Pacific, and includes ten genera: Acutotyphlops, Anilios, Asiatyphlops gen. nov., Cyclotyphlops, Grypotyphlops, Indotyphlops gen. nov., Malayotyphlops gen. nov., Ramphotyphlops, Sundatyphlops gen. nov., and Xerotyphlops gen. nov. Madatyphlopinae subfam. nov. occurs only in Madagascar and includes one genus: Madatyphlops gen. nov. Typhlopinae occurs in the New World and includes four genera: Amerotyphlops gen. nov., Antillotyphlops gen. nov., Cubatyphlops gen. nov., and Typhlops. Scolecophidians are the most ancient (deeply-branching) group of living snakes and their relationships track plate tectonics better than any other vertebrate group. Molecular data reveal large numbers of undescribed species, inferring that the true species diversity of these snakes is greatly underestimated.
\end{abstract}

Keywords: phylogeny, blindsnakes, Scolecophidia, Typhlopidae, systematics, biogeography, cryptic species.

\section{Introduction}

Scolecophidians are distributed on all continents except Antarctica but they are most diverse in tropical regions of continents and tropical islands (McDiarmid et al. 1999; Uetz \& Hošek 2013). These worm-like snakes have been neglected in nearly all aspects of vertebrate research. Approximately 400 described species are placed in five families: Anomalepididae (anomalepidids, 18 species), Leptotyphlopidae (threadsnakes, 116 species), Typhlopidae (typical blindsnakes, 257 species), Gerrhopilidae (Indo-Malayan blindsnakes, 16 species), and Xenotyphlopidae (round-nosed blindsnake, 1 species) (Adalsteinsson et al. 2009; Vidal et al. 2010; Uetz \& Hošek 2013). The Caribbean islands are unusual in having one of the largest blindsnake faunas in the world, numbering 44 species of typhlopids, with at least 25 additional undescribed species (see below).

This taxonomic update is a continuation of a series of systematic studies of scolecophidian snakes by our group (Hedges 1989; Hedges \& Thomas 1991; Hedges et al. 1992; Rabosky et al. 2004; Thomas \& Hedges 2007; 
Hedges 2008; Adalsteinsson et al. 2009; Vidal et al. 2010; Hedges 2011; Marin et al. 2013a; Marin et al. 2013b; Wegener et al. 2013). Until recently (Adalsteinsson et al. 2009; Vidal et al. 2010), there was no published tree of the evolutionary relationships of scolecophidians, which is remarkable for a major group of vertebrates. One reason for the slow progress in the systematics of these snakes has been the paucity of useful morphological characters, not uncommon in burrowing reptiles with conservative body plans and often an impetus for exploring molecular data (Vidal et al. 2008). The use of molecular markers in scolecophidians has proven especially valuable in revealing cryptic species (Hedges \& Thomas 1991; Aplin \& Donnellan 1993; Rabosky et al. 2004; Thomas \& Hedges 2007; Hedges 2008; Kornilios et al. 2011; Marin et al. 2013b) as well as discovering that some species are not valid (Wegener et al. 2013).

In recent years, two of us (SBH, NV) have expanded efforts to improve the systematics of scolecophidian snakes, globally, by assembling tissue samples with the help of collaborators working in Africa (W. Branch), Madagascar (M. Vences), and Australia (K. P. Aplin, S. C. Donnellan, P. Doughty, and M. N. Hutchinson) as well as donations of samples from persons working in other regions. This international effort has resulted in the generation of 2,414 DNA sequences from 11 nuclear and mitochondrial genes, all of which have been deposited in GenBank. Our analyses of these data have greatly refined the taxonomy of both of the major groups, the threadsnakes (Adalsteinsson et al. 2009) and blindsnakes (Vidal et al. 2010), as well as their biogeographic history (Vidal et al. 2010; Marin et al. 2013a). For example, it has led to the recognition and description of 10 new family group taxa and five new genus group taxa (Adalsteinsson et al. 2009; Vidal et al. 2010; Hedges 2011). However, these ongoing revisionary efforts have required continued sequence data collection and assembly of useful and relevant morphological characters. We have collected more than 1000 new DNA sequences of scolecophidians, not included in our previous studies, and all of these data, including morphological data, have helped to define supraspecific clades within Typhlopidae unrecognized previously. Although our intent has been to release this revisionary work in a single monograph, we are releasing the pertinent taxonomic information here. Hence, this current work, focused on Typhlopidae, should be viewed more as a preliminary taxonomic synopsis of a larger and more comprehensive work to come later. Here, we present 489 new DNA sequences, and molecular phylogenies that include at least 150 species (described and undescribed) of snakes in this family, along with a revised taxonomic framework and descriptions of new supraspecific taxa.

\section{Materials and Methods}

Data collection. Three separate molecular datasets were built for this study. We expanded our worldwide scolecophidian dataset, called here dataset A (Vidal et al. 2010), our Australian typhlopid dataset, dataset B (Marin et al. 2013a; Marin et al. 2013b), and built an expanded West Indian typhlopid dataset, dataset C. Each of the three datasets includes published and new sequences. However, for dataset $B$, all of the new sequences are from the samples used in Marin et al. (2013a, b); to avoid confusion, we list those samples and localities in Appendix 1. GenBank accession numbers for the 182 new sequences used in dataset B are KF992951-KF993132. For the previously published sequences (and museum accession numbers and localities) used in datasets A and C, see Vidal et al. (2010). GenBank accession numbers for the 105 new sequences used in dataset A are: KF992846-KF992950. GenBank accession numbers for the 202 new sequences used in dataset C are KF993133-KF993334. See Appendix 1 for details of the new samples used in the molecular analyses.

Laboratory methods. DNA extraction was performed as described in Winnepenninckx et al. (1993) or with the DNeasy Tissue Kit from Qiagen. The following genes were used: BDNF, RAG1, BMP2, NT3, and AMEL for dataset A (Vidal et al. 2010); cytochrome b, PRLR, BDNF, BMP2, 12S and 16S rRNA for dataset B (Marin et al. 2013a; Marin et al. 2013b); cytochrome b, ND2, 12 S and 16S rRNA, PRLR, BDNF, RAG1, BMP2, NT3, and AMEL for dataset C. Amplification and sequencing was performed using primers from our previous scolecophidian studies (Adalsteinsson et al. 2009; Vidal et al. 2010; Marin et al. 2013a; Marin et al. 2013b) with the exception of those for 12S rRNA, $16 \mathrm{~S}$ 
rRNA, and ND2 (always 5' to 3'): NV-12L2-AAA GCA WRG CAC TGA ARA TGC TWA GAT, NV-12H11-CAC TTT CCA GTA CGC TTA CCA TGT TAC G, JM-12L3-GGG GTG ACG GGC GGT GTG T, JM-12H13-AGT ATA CAT GCA AGW CTC, for the 12S rRNA; L2510-CGC CTG TTT ATC AAA AAC AT (Palumbi et al. 1991), H3056-CTC CGG TCT GAA CTC AGA TCA CGT AGG (Hedges 1994), for the 16S rRNA. For ND2, we designed 5 sets of primers: NDF1/NDR1, ND2F1/ND2R3, ND2F4/ ND2R4, ND2F5/ND2R5, and ND2F6/ND2R6. Those primers are: ND2F1-CAG CTA AAT AAG CTM TCG GGC CCA TAC C, NDR1-ACT TCT GGT ACT CAR AAR TG, ND2R3-GCT TTG AAG GCY SCT GGT TTA, ND2F4-ACT GGA TTY WTR CCW AAA TGA AT, ND2R4-GAT CCG ATG TCT TTA ATR GTT, ND2F5-CTA AAY CAA ACA CAA CTM CGA, ND2R5-GAT CCG ATG TCT TTA ATR GTT, ND2F6-CCA TTT CAC TTC TGA GTR CCA GAA GT, and ND2R6-TCG KAG TTG TGT TTG RTT TAG. For all genes, DNA amplification was performed in a $21 \mu \mathrm{L}$ final volume containing $1 \mu \mathrm{L}$ DMSO, $0.8 \mu \mathrm{L}$ of dNTP $(6.6 \mathrm{mM}), 0.12 \mu \mathrm{L}$ of Taq DNA polymerase (MP Biomedicals or Qiagen), using $2.5 \mu \mathrm{L}$ of the buffer provided by the manufacturer, $100{\mathrm{u} . \mathrm{ml}^{-1}}^{-1}$ and $0.32 \mu \mathrm{L}$ of each primer at $10 \mathrm{pM}$, and $1 \mu \mathrm{L}$ of DNA extract. The PCR conditions were: an initial denaturation for $3 \mathrm{~min}$ at $94^{\circ} \mathrm{C}$ followed by 40 cycles $\left(3 \mathrm{~min}\right.$ at $94^{\circ} \mathrm{C}, 40 \mathrm{~s}$ at $50^{\circ} \mathrm{C}, 1 \mathrm{~min}$ at $72^{\circ} \mathrm{C}$ ) and a final elongation at $72^{\circ} \mathrm{C}$ for 10 min, using a PCR System 2700 thermocycler (Applied Biosystems).

Both complementary strands of the PCR products were sequenced at Pennsylvania State University and Genoscope (http://www.genoscope.fr). The two strands obtained for each sequence, and multiple sequences, were combined and aligned using the BioEdit Sequence Alignment Editor program (Hall 1999) and alignment tools in MEGA5 (Tamura et al. 2011). Any difference between the two strands was coded as undetermined. Amino acid translations were used and alignment was straightforward for all nuclear genes. Alignment was also straightforward for $12 \mathrm{~S}$ and $16 \mathrm{~S}$ rRNA as the longest gap had a length of $4 \mathrm{bp}$. In all analyses, gaps were treated as missing data. Alignments can be obtained from Nicolas Vidal. For dataset A, alignments resulted in 630 BDNF sites, 516 RAG1 sites, 591 BMP2 sites, 639 NT3 sites, and 375 AMEL sites (total: 2751 sites from 5 genes for 127 taxa). For dataset $B$, alignments resulted in 678 cytochrome b sites, 483 PRLR sites, 669 BDNF sites, 588 BMP2 sites, 717 12S rRNA sites, and $40616 \mathrm{~S}$ rRNA sites (total: 3541 sites from 6 genes for 94 taxa). For dataset $C$, alignments resulted in 1107 cytochrome b sites, 573 16S rRNA sites, 383 12S rRNA sites, 909 ND2 sites, 552 PRLR sites, 630 BDNF sites, 516 RAG1 sites, 588 BMP2 sites, 639 NT3 sites, and 372 AMEL sites (total: 6269 sites from 10 genes for 54 taxa).

Phylogenetic analyses. We built all phylogenies using Maximum Likelihood (ML) and Bayesian methods of inference (BI). ML analyses were performed with RAxML 7.2.7 (Stamatakis 2006; Stamatakis et al. 2008), and Bayesian analyses were performed with MrBayes 3.1.2 (Ronquist \& Huelsenbeck 2003). For all datasets, we first analyzed each gene separately to check topological congruence before combining them in our final datasets. The partitioning strategy was the same for all three datasets: first codon positions of combined nuclear genes, second codon positions of combined nuclear genes, third codon positions of combined nuclear genes, first codon positions of combined mitochondrial genes, second codon positions of combined mitochondrial genes, third codon positions of combined mitochondrial genes, and combined $12 \mathrm{~S}$ and $16 \mathrm{~S}$ rRNA treated as one partition, with a GTR $+\mathrm{I}+\mathrm{G}$ model applied to each partition for Bayesian analyses and a GTR + G model applied to each partition for RAxML analyses as recommended in Stamatakis (2006).

For Bayesian analyses, four simultaneous Markov chains were run for 5 million generations, sampling every 100 generations, and discarding all samples during a 500,000 generation burnin-in period. Convergence of the Bayesian runs was examined by plots of $\ln L$ scores and low standard deviation of split frequencies as implemented in MrBayes 3.1.2. Two independent Bayesian runs were performed as an additional check that the chains mixed well and converged. For the ML analyses, we performed 1,000 bootstrap replicates, using the GTRCAT option. The resulting ML and Bayesian trees were visualized with FigTree 1.3.1. (http://tree.bio.ed.ac.uk/software/figtree/).

Morphological data. One of us (SBH) has collected and examined nearly every species of typhlopid snake from Caribbean islands, and has borrowed and examined comparative material from museums (e.g., Hedges \& Thomas 1991; Thomas \& Hedges 2007). However, most of our morphological data (Tables 1-2), globally, are from the literature, including species descriptions (Table 3), summary articles, and illustrations of head scalation. We report classical characters used in the group, but also with a focus on their diagnostic ability. For example, postnasal 
Table 1. Morphological variation in the species of typhlopid snakes. Numbered columns correspond to the 20 numbered characters in the diagnoses. States separated by commas are equally frequent; those in parentheses are uncommon. Abbreviations: (1, eye), d (distinct), i (indistinct ); ( 2 , snout), b (beaked), r (rounded), a (acuminate); (3, head scales), c (circular), nc (non circular); (4, frontorostral), p (present), a (absent); (5, nasal division), c (complete), i (incomplete); (6, nasal suture origin), 1 (supralabial 1), 2 (supralabial 2), s1 (suture 1 \& 2), s2 (suture 2 \& preocular), r (rostral), p (preocular); (7, subocular or subpreocular scales), p (present), a, (absent); (8a, postocular range); (8b, postocular average); (9, preocular-labial contact), 0 (no contact), $0 \mathrm{i}$ (intercalary scale), 0 s (subpreocular), otherwise single supralabial scales or range of scales contacted are listed; (10a, midbody scale rows, range); (10b, midbody scale rows, average); (11, scale row reduction), p (present), a (absent); (12a, total middorsal scale rows, range); (12b, total middorsal scale rows, average); (13a, caudal scales, range); (13b, caudal scales, average); (14a, total length in $\mathrm{mm}$, range of species maximums); (14b, total length, average of species maximums); (15a, body shape = total length/width, range); (15b, body shape, average); (16a, total length/tail length , range); (16b, total length/tail length, average); (17, dorsal ground color); (18, ventral ground color); (19, dorsal-ventral color difference), 0 (no difference = unicolor), 1 (dorsum darker = bicolor); (20, pattern), bl (blotched or marbled), br (bars), dl (dorsal lines), juv (juveniles), nk (neck band or collar), rt (reticulated), sp (spots or speckling), tr (tail rings), un (unpatterned), $\mathrm{v}$ (variable), ven (venter), vl (ventral lines); $\mathrm{n} / \mathrm{a}$ (not available). Color abbreviations: bk (black), bl (blue), bg (beige), bn (brown), cr (cream), dk (dark), gd (gold), gn (green), gy (gray), Iv (lavender), oc (ochre), ol (olive), or (orange), pk (pink or pale red), pl (pale), pu (purple), rd (red), tn (tan), um (umber), un (unpigmented), wt (white), yl (yellow).

\begin{tabular}{|c|c|c|c|c|c|c|c|c|c|c|c|}
\hline & 1 & 2 & 3 & 4 & 5 & 6 & 7 & $8 a$ & 9 & $10 a$ & 11 \\
\hline \multicolumn{12}{|l|}{ AFROTYPHLOPINAE } \\
\hline Afrotyphlops angolensis & d & $r$ & $\mathrm{nc}$ & a & $\mathrm{i}$ & 1 & a & 3 & $2-3$ & $24-36$ & $p$ \\
\hline Afrotyphlops anomalus & $d$ & $b$ & nc & a & $\mathrm{i}$ & $r$ & a & $4-7 ?$ & $1-3$ & $29-32$ & $\mathrm{p}$ \\
\hline Afrotyphlops bibronii & $d$ & $r$ & nc & a & $\mathrm{i}$ & 1 & a & $3-4$ & 2 & $30-34$ & $\mathrm{p}$ \\
\hline Afrotyphlops blanfordii & $d$ & $r$ & nc & a & $\mathrm{i}$ & 1 & a & 4 & $2-3$ & $28-32$ & $\mathrm{p}$ \\
\hline Afrotyphlops brevis & $d$ & b & nc & a & $\mathrm{i}$ & 2 & a & $3-4$ & $2-3$ & $29-38$ & $\mathrm{p}$ \\
\hline Afrotyphlops calabresii & $d$ & a & nc & a & $\mathrm{i}$ & 2 & a & 2 & $2-3$ & $20-22$ & a \\
\hline Afrotyphlops comorensis & $d$ & $r$ & nc & a & $\mathrm{i}$ & 2 & a & $2-4$ & $2-3$ & $20-22$ & $\mathrm{p}$ \\
\hline Afrotyphlops congestus & $d$ & $r$ & nc & a & $\mathrm{i}$ & 1 & a & 3 & $2-3$ & $24-30$ & $\mathrm{p}$ \\
\hline Afrotyphlops cuneirostris & $d$ & a & nc & a & i? & 2 & a & 2 & $2-3$ & $20-24$ & $a(p)$ \\
\hline Afrotyphlops decorosus & $d$ & $r$ & nc & a & i? & 1 & a & 4 & $2-3$ & 24 & $a$ \\
\hline Afrotyphlops elegans & $d$ & $r$ & nc & $\mathrm{a}$ & $\mathrm{i}, \mathrm{c}$ & 1 & a & 3 & $2-3$ & $18-20$ & $n / a$ \\
\hline Afrotyphlops fornasinii & $d$ & $r$ & nc & $\mathrm{a}$ & $c, i$ & 1 & a & 4 & 2 & $22-26$ & $p, a$ \\
\hline Afrotyphlops gierrai & $d$ & $r$ & nc & $\mathrm{a}$ & $\mathrm{i}$ & 1 & $\mathrm{p}$ & 4 & $0 \mathrm{i}$ & $26-28$ & $p, a$ \\
\hline Afrotyphlops jubanus & $d$ & $\mathrm{~b}$ & nc & a & $\mathrm{i}$ & 1 & a & 4 & $2-3$ & 24 & $\mathrm{p}$ \\
\hline Afrotyphlops kaimosae & $d$ & b & nc & a & $\mathrm{i}$ & 1 & $a$ & 5 & 0 & 28 & $a$ \\
\hline Afrotyphlops liberiensis & $d$ & $r$ & nc & $\mathrm{a}$ & $\mathrm{n} / \mathrm{a}$ & $\mathrm{n} / \mathrm{a}$ & $\mathrm{n} / \mathrm{a}$ & $\mathrm{n} / \mathrm{a}$ & $\mathrm{n} / \mathrm{a}$ & $\mathrm{n} / \mathrm{a}$ & $\mathrm{n} / \mathrm{a}$ \\
\hline Afrotyphlops lineolatus & $d$ & $r$ & nc & $\mathrm{a}$ & $\mathrm{i}$ & 1 & $\mathrm{a}$ & $4-5$ & $2-3$ & $26-30$ & $p$ \\
\hline Afrotyphlops mucruso & $d$ & b & nc & a & $\mathrm{i}$ & 1 & a & $6-7$ & $2-3,2$ & $30-40$ & $\mathrm{p}$ \\
\hline Afrotyphlops nanus & $d$ & $r$ & nc & a & $\mathrm{i}$ & 1 & a & $2-3$ & 2 & 30 & $\mathrm{p}$ \\
\hline Afrotyphlops nigrocandidus & $d$ & $r$ & nc & a & $\mathrm{i}$ & 1 & $\mathrm{p}(\mathrm{a})$ & $\mathrm{n} / \mathrm{a}$ & $0 \mathrm{i}$ & $28-34$ & $\mathrm{p}$ \\
\hline Afrotyphlops obtusus & $d$ & r & nc & a & $\mathrm{i}$ & 1 & $a$ & $>3$ & $2-3$ & $22-26$ & $\mathrm{p}$ \\
\hline Afrotyphlops platyrhynchus & $d$ & r & nc & a & $\mathrm{i}$ & 2 & a & 3 & $2-3$ & 24 & $\mathrm{p}$ \\
\hline Afrotyphlops punctatus & $d$ & r & nc & a & $\mathrm{i}$ & 1 & a & 4 & $2-3$ & $28-32$ & $\mathrm{p}(\mathrm{a})$ \\
\hline Afrotyphlops rondoensis & $d$ & $r$ & nc & $\mathrm{a}$ & $\mathrm{i}$ & 1 & a & $3-4$ & 2 & $22-26$ & $\mathrm{p}(\mathrm{a})$ \\
\hline Afrotyphlops schlegelii & $d$ & $b$ & nc & a & $\mathrm{i}$ & 1 & a & $4-6$ & $2-3$ & $32-45$ & $p$ \\
\hline Afrotyphlops schmidti & $d$ & $r$ & nc & a & $\mathrm{i}$ & 1 & a & 4 & $2-3$ & $22-26$ & $\mathrm{p}$ \\
\hline Afrotyphlops steinhausi & $d$ & r & nc & a & $\mathrm{i}$ & 1 & a & $3-4$ & $1-2$ & $26-28$ & $\mathrm{p}$ \\
\hline Afrotyphlops tanganicanus & $d$ & r & nc & a & $\mathrm{i}$ & 1 & a & 3 & $2-3$ & $21-24$ & $\mathrm{p}$ \\
\hline Afrotyphlops usambaricus & $d$ & $r$ & nc & a & $\mathrm{i}$ & $\mathrm{r} / 1 ?$ & $a$ & 4 & $2-3$ & $26-28$ & $\mathrm{p}$ \\
\hline Letheobia acutirostrata & $\mathrm{i}$ & $\mathrm{n} / \mathrm{a}$ & nc & a & $\mathrm{n} / \mathrm{a}$ & $\mathrm{n} / \mathrm{a}$ & $\mathrm{n} / \mathrm{a}$ & $\mathrm{n} / \mathrm{a}$ & $\mathrm{n} / \mathrm{a}$ & $\mathrm{n} / \mathrm{a}$ & $\mathrm{n} / \mathrm{a}$ \\
\hline Letheobia angeli & $\mathrm{i}$ & $\mathrm{n} / \mathrm{a}$ & nc & a & $\mathrm{n} / \mathrm{a}$ & 2 & $\mathrm{n} / \mathrm{a}$ & $\mathrm{n} / \mathrm{a}$ & $\mathrm{n} / \mathrm{a}$ & $\mathrm{n} / \mathrm{a}$ & $\mathrm{n} / \mathrm{a}$ \\
\hline Letheobia caeca & $\mathrm{i}$ & $\mathrm{b}$ & nc & a & $c$ & $2(r, 1, s 1)$ & $\mathrm{p}$ & 4 & $2-3,3$ & $22-26$ & $\mathrm{n} / \mathrm{a}$ \\
\hline Letheobia coecata & $\mathrm{i}$ & $r$ & nc & a & c & 2 & a & $2-3 ?$ & 3 & $18-20$ & $\mathrm{n} / \mathrm{a}$ \\
\hline Letheobia crossii & $\mathrm{i}$ & b & nc & a & c & 2 & a & $3-4$ & $2-3 ?$ & $22-24$ & $\mathrm{p}$ \\
\hline Letheobia debilis & $\mathrm{i}$ & b & nc & a & $\mathrm{i}$ & s1? & $\mathrm{p}$ & 4 & 2 & $18-20$ & $p, a$ \\
\hline Letheobia episcopa & $\mathrm{i}$ & b & nc & a & c & 2 & $\mathrm{p}$ & $4-6 ?$ & $2-4$ & 20 & $\mathrm{a}$ \\
\hline Letheobia erythraea & $\mathrm{i}$ & b & nc & a & c & 2 & $\mathrm{p}$ & $3-4 ?$ & $2-3$ & $20-23$ & $\mathrm{p}$ \\
\hline Letheobia feae & $\mathrm{i}$ & b & nc & a & c & 2 & a & 2 & $2-3$ & $20-22$ & $p, a$ \\
\hline Letheobia gracilis & $\mathrm{i}$ & $b$ & nc & a & $c, i ?$ & s1? & $\mathrm{p}$ & 4 & $2-3$ & 22 & $\mathrm{a}$ \\
\hline
\end{tabular}


Letheobia graueri

Letheobia kibarae

Letheobia largeni

Letheobia leucosticta

Letheobia lumbriciformis

Letheobia manni

Letheobia newtoni

Letheobia pallida

Letheobia pauwelsi

Letheobia pembana

Letheobia praeocularis

Letheobia rufescens

Letheobia simonii

Letheobia somalica

Letheobia stejnegeri

Letheobia sudanensis

Letheobia swahilica

Letheobia toritensis

Letheobia uluguruensis

Letheobia wittei

Letheobia zenkeri

Rhinotyphlops ataeniatus

Rhinotyphlops boylei

Rhinotyphlops lalandei

Rhinotyphlops leucocephalus

Rhinotyphlops schinzi

Rhinotyphlops scortecci

Rhinotyphlops unitaeniatus

\section{ASIATYPHLOPINAE}

Acutotyphlops banaorum

Acutotyphlops infralabialis

Acutotyphlops kunuaensis

Acutotyphlops solomonis

Acutotyphlops subocularis

Anilios affinis

Anilios ammodytes

Anilios aspinus

Anilios australis

Anilios batillus

Anilios bicolor

Anilios bituberculatus

Anilios broomi

Anilios centralis

Anilios chamodracaena

Anilios diversus

Anilios endoterus

Anilios erycinus

Anilios ganei

Anilios grypus

Anilios guentheri

Anilios hamatus

Anilios how

Anilios kimberleyensis

Anilios leptosomus

Anilios leucoproctus

Anilios ligatus

Anilios longissimus

$\begin{array}{llcc}\mathrm{nc} & \mathrm{a} & \mathrm{i} & 1, \mathrm{~s} 1,2 \\ \mathrm{nc} & \mathrm{a} & \mathrm{i} & 1 \\ \mathrm{nc} & \mathrm{a} & \mathrm{c} & 2 \\ \mathrm{nc} & \mathrm{a} & \mathrm{c}, \mathrm{i} ? & 2 \\ \mathrm{nc} & \mathrm{a} & \mathrm{i} & 2 \\ \mathrm{nc} & \mathrm{a} & \mathrm{i} & 2 \\ \mathrm{nc} & \mathrm{a} & \mathrm{c}, \mathrm{i} ? & 2 \\ \mathrm{nc} & \mathrm{a} & \mathrm{i} & 2 \\ \mathrm{nc} & \mathrm{a} & \mathrm{c} & 1 \\ \mathrm{nc} & \mathrm{a} & \mathrm{n} / \mathrm{a} & 2 \\ \mathrm{nc} & \mathrm{a} & \mathrm{c} & 2 \\ \mathrm{nc} & \mathrm{a} & \mathrm{i} & 2 \\ \mathrm{nc} & \mathrm{a} & \mathrm{i}, \mathrm{c} & 1 \\ \mathrm{nc} & \mathrm{a} & \mathrm{c} & \mathrm{s} 1 ? \\ \mathrm{nc} & \mathrm{a} & \mathrm{c} & \mathrm{s} 1 ? \\ \mathrm{nc} & \mathrm{a} & \mathrm{i} & 2 \\ \mathrm{nc} & \mathrm{a} & \mathrm{c} & 2 \\ \mathrm{nc} & \mathrm{a} & \mathrm{i} & 1 \\ \mathrm{nc} & \mathrm{a} & \mathrm{c} & 2 \\ \mathrm{nc} & \mathrm{a} & \mathrm{i} & 2 \\ \mathrm{nc} & \mathrm{a} & \mathrm{c} & \mathrm{p} \\ \mathrm{nc} & \mathrm{a} & \mathrm{c} & 2 \\ \mathrm{nc} & \mathrm{a} & \mathrm{i} & 2 \\ \mathrm{nc} & \mathrm{a} & \mathrm{i} & 1 \\ \mathrm{nc} & \mathrm{a} & \mathrm{i} & 2 \\ \mathrm{nc} & \mathrm{a} & \mathrm{i} & 1 \\ \mathrm{nc} & \mathrm{a} & \mathrm{c} & 2 \\ \mathrm{nc} & \mathrm{a} & \mathrm{c} & 2\end{array}$

$\begin{array}{ccccc}\mathrm{p} & 2 & 2-3 & 24 & \mathrm{a} \\ \mathrm{p} & 4 & 2-3 & 24 & \mathrm{p} \\ \mathrm{p} & 4 & 2-3 & 22 & \mathrm{p} \\ \mathrm{a} & 3 & 2-3 & 22 & \mathrm{p} \\ \mathrm{p} & 4 & 2-3 & 18 & \mathrm{a}(\mathrm{p}) \\ \mathrm{p} & 4 & 2-3 & 26 & \mathrm{n} / \mathrm{a} \\ \mathrm{a} & 3 & 3 & 26-28 & \mathrm{p} \\ \mathrm{p} & 4-7 ? & 2-3 & 22 & \mathrm{p}(\mathrm{a}) \\ \mathrm{p} & 3-4 ? & 2-3 & 22 & \mathrm{a} \\ \mathrm{p} & \mathrm{n} / \mathrm{a} & \mathrm{n} / \mathrm{a} & 24 & \mathrm{p} \\ \mathrm{a} & 3 ? & 0 & 24-26 & \mathrm{p} \\ \mathrm{p} & 3-5 & 2 & 20 & \mathrm{p}, \mathrm{a} \\ \mathrm{a} & 2-3 & 2-3 ? & 20-21 & \mathrm{p}, \mathrm{a} \\ \mathrm{a} & 3-6 & 2-3 & 24-30 & \mathrm{p}, \mathrm{a} \\ \mathrm{a} & 3 & 2 & 26 & \mathrm{p} \\ \mathrm{p} & 3 & 2-3 & 24 & \mathrm{p}, \mathrm{a} \\ \mathrm{p} & 4 & 2-3 & 22 & \mathrm{p}(\mathrm{a}) \\ \mathrm{p} & 3 & 2-3 & 22-24 & \mathrm{p} \\ \mathrm{p} & 5 & 2-3 & 20-22 & \mathrm{p} \\ \mathrm{p} & 4 & 2 & 20 & \mathrm{a} \\ \mathrm{p} & 2 & 0 \mathrm{~s} & 18 & \mathrm{n} / \mathrm{a} \\ \mathrm{p} & 4 & 2-3 & 24 & \mathrm{p}(\mathrm{a}) \\ \mathrm{a} & 5 & 2-3 & 24-28 & \mathrm{p} \\ \mathrm{a} & 2 & 2,2-3 & 26-34 & \mathrm{p}(\mathrm{a}) \\ \mathrm{a} & 3 & 2-3 & 24 & \mathrm{n} / \mathrm{a} \\ \mathrm{a} & 4-6 & 2 & 22-26 & \mathrm{a} \\ \mathrm{p} & 4-5 & 2-4 & 23-25 & \mathrm{p}(\mathrm{a}) \\ \mathrm{p} & 6 & 2-3 & 24 & \mathrm{p}\end{array}$

$\begin{array}{llcc}\mathrm{nc} & \mathrm{p} & \mathrm{i} & 2 \\ \mathrm{nc} & \mathrm{p} & \mathrm{i} ? & 1 \\ \mathrm{nc} & \mathrm{p} & \mathrm{i} & 2 \\ \mathrm{nc} & \mathrm{p} & \mathrm{i} & 2 \\ \mathrm{nc} & \mathrm{p} & \mathrm{i} & 2 \\ \mathrm{nc} & \mathrm{a} & \mathrm{c} & 2 \\ \mathrm{nc} & \mathrm{a} & \mathrm{c}, \mathrm{i} & \mathrm{p} \\ \mathrm{nc} & \mathrm{a} & \mathrm{i} & 2 \\ \mathrm{nc} & \mathrm{a} & \mathrm{i} & 2(\mathrm{~s} 1, \mathrm{~s} 2, \mathrm{p}) \\ \mathrm{nc} & \mathrm{a} & \mathrm{c} & 2 \\ \mathrm{nc} & \mathrm{a} & \mathrm{i} & \mathrm{s} 2 ? \\ \mathrm{nc} & \mathrm{a} & \mathrm{i} & 2 \\ \mathrm{nc} & \mathrm{a} & \mathrm{i}, \mathrm{c} & 2 \\ \mathrm{nc} & \mathrm{a} & \mathrm{i} & 2 \\ \mathrm{nc} & \mathrm{a} & \mathrm{i} & 2 \\ \mathrm{nc} & \mathrm{a} & \mathrm{c}, \mathrm{i} & \mathrm{p} \\ \mathrm{nc} & \mathrm{a} & \mathrm{i} & \mathrm{p} \\ \mathrm{nc} & \mathrm{a} & \mathrm{c} & 1 \\ \mathrm{nc} & \mathrm{a} & \mathrm{c} & 2 \\ \mathrm{nc} & \mathrm{a} & \mathrm{i}, \mathrm{c} & 2(\mathrm{p}, 1, \mathrm{~s} 2) \\ \mathrm{nc} & \mathrm{a} & \mathrm{i} & 2 \\ \mathrm{nc} & \mathrm{a} & \mathrm{i} & 2(\mathrm{p}, 1, \mathrm{~s} 1) \\ \mathrm{nc} & \mathrm{a} & \mathrm{c} & 2 \\ \mathrm{nc} & \mathrm{a} & \mathrm{i} & 2 \\ \mathrm{nc} & \mathrm{a} & \mathrm{c}, \mathrm{i} & 2 \\ \mathrm{nc} & \mathrm{a} & \mathrm{i} & 2 \\ \mathrm{nc} & \mathrm{a} & \mathrm{i} & 1 \\ \mathrm{nc} & \mathrm{a} & \mathrm{i} & 2\end{array}$


Anilios margaretae

Anilios micrommus

Anilios minimus

Anilios nema

Anilios nigrescens

Anilios nigroterminatus

Anilios pilbarensis

Anilios pinguis

Anilios proximus

Anilios robertsi

Anilios silvia

Anilios splendidus

Anilios torresianus

Anilios tovelli

Anilios troglodytes

Anilios unguirostris

Anilios waitii

Anilios wiedii

Anilios yampiensis

Anilios yirrikalae

Asiatyphlops bothriorhynchus

Asiatyphlops diardii

Asiatyphlops fuscus

Asiatyphlops giadinhensis

Asiatyphlops klemmeri

Asiatyphlops koshunensis

Asiatyphlops leucomelas

Asiatyphlops muelleri

Asiatyphlops oatesii

Asiatyphlops roxaneae

Asiatyphlops siamensis

Asiatyphlops tenuicollis

Asiatyphlops trangensis

Cyclotyphlops deharvengi

Grypotyphlops acutus

Indotyphlops ahsanai

Indotyphlops albiceps

Indotyphlops braminus

Indotyphlops exiguus

Indotyphlops filiformis

Indotyphlops fletcheri

Indotyphlops hypsobothrius

Indotyphlops jerdoni

Indotyphlops khoratensis

Indotyphlops lankaensis

Indotyphlops lazelli

Indotyphlops loveridgei

Indotyphlops madgemintonae

Indotyphlops malcolmi

Indotyphlops meszoelyi

Indotyphlops ozakiae

Indotyphlops pammeces

Indotyphlops porrectus

Indotyphlops schmutzi

Indotyphlops tenebrarum

Indotyphlops veddae

Indotyphlops violaceus

Malayotyphlops canlaonensis d $\quad r \quad n c \quad$ a $\quad$ i

$\begin{array}{lllll}\text { a } & \mathrm{n} / \mathrm{a} & 2-3 & 18 & \mathrm{n} / \mathrm{a}\end{array}$

d $\quad r \quad n c \quad$ a $c$

$\begin{array}{llllll}d & r & n c & a & i & 2\end{array}$

d

d

d $b \quad n c \quad a \quad c$

d b nc a

d

d

d

d

(a)

d

d

d

d

d

d

d

d

d

d

d

d

d

d

d

d

d

d

,

dis

di

di

d

d

d

d

id

i

d

id

d

d

d

d


Malayotyphlops castanotus Malayotyphlops collaris

Malayotyphlops hypogius

Malayotyphlops koekkoeki

Malayotyphlops kraali

Malayotyphlops luzonensis

Malayotyphlops manilae

Malayotyphlops ruber

Malayotyphlops ruficauda

Ramphotyphlops acuticauda

Ramphotyphlops adocetus

Ramphotyphlops angusticeps

Ramphotyphlops becki

Ramphotyphlops conradi

Ramphotyphlops cumingii

Ramphotyphlops depressus

Ramphotyphlops exocoet

Ramphotyphlops flaviventer

Ramphotyphlops hatmaliyeb

Ramphotyphlops lineatus

Ramphotyphlops lorenzi

Ramphotyphlops mansuetus

Ramphotyphlops marxi

Ramphotyphlops melanocephalus

Ramphotyphlops multilineatus

Ramphotyphlops olivaceus

Ramphotyphlops similis

Ramphotyphlops suluensis

Ramphotyphlops supranasalis

Ramphotyphlops willeyi

Sundatyphlops polygrammicus

Xerotyphlops etheridgei

Xerotyphlops socotranus

Xerotyphlops vermicularis

Xerotyphlops wilsoni

MADATYPHLOPINAE

Madatyphlops andasibensis

Madatyphlops arenarius

Madatyphlops boettgeri

Madatyphlops decorsei

Madatyphlops domerguei

Madatyphlops madagascariensis

Madatyphlops microcephalus

Madatyphlops mucronatus

Madatyphlops ocularis

Madatyphlops rajeryi

Madatyphlops reuteri

\section{TYPHLOPINAE}

Amerotyphlops amoipira

Amerotyphlops brongersmianus

Amerotyphlops costaricensis

Amerotyphlops lehneri

Amerotyphlops microstomus

Amerotyphlops minuisquamus

Amerotyphlops paucisquamus

Amerotyphlops reticulatus

Amerotyphlops stadelmani

intis

$d$
$d$
$d$
$i$
$d$
$d$
$d$
$d$
$d$
$d$
$d$
$d$
$d$
$d$
$d$
$d$
$d$
$d$
$d$
$d$
$d$
$d$
$d$
i
$d$
$d$
$d$
$d$
$d$
$d$
$d$
$d$
$d$

$\begin{array}{llll}\mathrm{nc} & \mathrm{a} & \mathrm{i} & 2 \\ \mathrm{nc} & \mathrm{a} & \mathrm{i} & 2 \\ \mathrm{nc} & \mathrm{a} & \mathrm{i} & 2 \\ \mathrm{nc} & \mathrm{a} & \mathrm{i} & 1 \\ \mathrm{nc} & \mathrm{a} & \mathrm{c} & 2 \\ \mathrm{nc} & \mathrm{a} & \mathrm{c} & 2 \\ \mathrm{nc} & \mathrm{a} & \mathrm{i} & 2 \\ \mathrm{nc} & \mathrm{a} & \mathrm{i} & 2 \\ \mathrm{nc} & \mathrm{a} & \mathrm{i} & 2 \\ \mathrm{nc} & \mathrm{a} & \mathrm{c} & 2 \\ \mathrm{nc} & \mathrm{a} & \mathrm{c} & 2 \\ \mathrm{nc} & \mathrm{a} & \mathrm{c} & 1 \\ \mathrm{nc} & \mathrm{a} & \mathrm{c} & 2 \\ \mathrm{nc} & \mathrm{a} & \mathrm{c} & 2 \\ \mathrm{nc} & \mathrm{a} & \mathrm{c} & 2 \\ \mathrm{nc} & \mathrm{a} & \mathrm{c} & 2 \\ \mathrm{nc} & \mathrm{a} & \mathrm{i} & 2 \\ \mathrm{nc} & \mathrm{a} & \mathrm{l}, \mathrm{c} & 2 \\ \mathrm{nc} & \mathrm{a} & \mathrm{c} & 2 \\ \mathrm{nc} & \mathrm{a} & \mathrm{i} & 1 \\ \mathrm{nc} & \mathrm{a} & \mathrm{i} & 1 \\ \mathrm{nc} & \mathrm{a} & \mathrm{c} & 2 \\ \mathrm{nc} & \mathrm{a} & \mathrm{c} & 2 \\ \mathrm{nc} & \mathrm{a} & \mathrm{i} & 2 \\ \mathrm{nc} & \mathrm{a} & \mathrm{i} & 1 \\ \mathrm{nc} & \mathrm{a} & \mathrm{c}, \mathrm{i} & \mathrm{s} 1 \\ \mathrm{nc} & \mathrm{a} & \mathrm{i} & 2 \\ \mathrm{nc} & \mathrm{a} & \mathrm{c} & 1 \\ \mathrm{nc} & \mathrm{a} & \mathrm{l}, \mathrm{c} & 2 \\ \mathrm{nc} & \mathrm{a} & \mathrm{c} & 2 \\ \mathrm{nc} & \mathrm{a} & \mathrm{l}, \mathrm{c} & 1, \mathrm{~s} 1,2 \\ \mathrm{nc} & \mathrm{a} & \mathrm{i} & 2 \\ \mathrm{nc} & \mathrm{a} & \mathrm{i} & 2 \\ \mathrm{nc} & \mathrm{a} & \mathrm{i} & 2 \\ \mathrm{nc} & \mathrm{a} & \mathrm{i} & 2 \\ & & \end{array}$

$\begin{array}{ccccc}\mathrm{a} & 2 & 3 & 26-28 & \mathrm{p} \\ \mathrm{a} & 3 & 3 & 26-28 & \mathrm{p} \\ \mathrm{a} & 2 & 2-3 & 24 & \mathrm{p} \\ \mathrm{a} & \mathrm{n} / \mathrm{a} & 2-3 & 26 & \mathrm{n} / \mathrm{a} \\ \mathrm{a} & 4 & 3 ? & 24-28 & \mathrm{p} \\ \mathrm{n} / \mathrm{a} & 2 & 2-3 & 26 & \mathrm{n} / \mathrm{a} \\ \mathrm{p} & 3 & 2 & 28 & \mathrm{n} / \mathrm{a} \\ \mathrm{n} / \mathrm{a} & \mathrm{n} / \mathrm{a} & 3 & 26 & \mathrm{n} / \mathrm{a} \\ \mathrm{a} & 4 & 3 & 26-30 & \mathrm{n} / \mathrm{a} \\ \mathrm{a} & 2 & 2-3 & 22-24 & \mathrm{n} / \mathrm{a} \\ \mathrm{a} & 3 & 2-3 & 22 & \mathrm{p} \\ \mathrm{a} ? & 3 & 2-3 & 20 & \mathrm{n} / \mathrm{a} \\ \mathrm{a} & 2 & 2-3 & 20 & \mathrm{n} / \mathrm{a} \\ \mathrm{a} & 1 & 2-3 & 20 & \mathrm{n} / \mathrm{a} \\ \mathrm{a} & 3 & 3 & 24-28 & \mathrm{n} / \mathrm{a} \\ \mathrm{a} & 1-3 ? & 2-3 & 22-24 & \mathrm{p}, \mathrm{a} \\ \mathrm{a} & 2 & 2-3 & 20 & \mathrm{n} / \mathrm{a} \\ \mathrm{a} & 2(3) & 2-3 & 22 & \mathrm{a}(\mathrm{p}) \\ \mathrm{a} & 2-3 & 2-3 & 22 & \mathrm{a} \\ \mathrm{a} & 2 & 0 & 22-24 & \mathrm{n} / \mathrm{a} \\ \mathrm{n} / \mathrm{a} & \mathrm{n} / \mathrm{a} & 2-3 & 22 & \mathrm{n} / \mathrm{a} \\ \mathrm{a} & 2 & 2-3 & 18 & \mathrm{n} / \mathrm{a} \\ \mathrm{a} & 3 & 2-3 & 30 & \mathrm{p} \\ \mathrm{a} & 1 & 0 & 18 & \mathrm{a} \\ \mathrm{a} & 2 & 2-3 & 20-23 & \mathrm{n} / \mathrm{a} \\ \mathrm{a} & 3 & 2-3 & 20-22 & \mathrm{a}(\mathrm{p}) \\ \mathrm{a} & 1 & 2-3 & 20 & \mathrm{p} \\ \mathrm{a} & 2 & 2-3 & 22 & \mathrm{a} \\ \mathrm{n} / \mathrm{a} & 2-3 & 2-3 & 22 & \mathrm{p}, \mathrm{a} \\ \mathrm{a} & 2 & 2-3 & 22 & \mathrm{n} / \mathrm{a} \\ \mathrm{a} & \mathrm{n} / \mathrm{a} & 2-3 & 22 & \mathrm{n} / \mathrm{a} \\ \mathrm{a} & 2 & 2-3 & 24 & \mathrm{p} \\ \mathrm{p} & \mathrm{n} / \mathrm{a} & 2-3 & 24 & \mathrm{p}\end{array}$

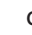

$\frac{9}{9}$

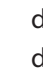

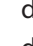

d

d

d

$\begin{array}{llc}\text { nc } & \text { a } & \text { i } \\ n c & a & c, i ? \\ n c & a & i \\ n c & a & i \\ n c & a & i \\ n c & a & i \\ n c & a & i \\ n c & a & i \\ n c & a & c \\ n c & a & i \\ n c & a & i\end{array}$

2
2
2
2
2
2
2
2
2
2
2

$\begin{array}{cccc}3(2) & 2-3 & 26 & \mathrm{p} \\ 2-3 & 2-3 & 20-24 & \mathrm{n} / \mathrm{a} \\ 2 & 2-3 & 20-22 & \mathrm{n} / \mathrm{a} \\ 2-3 & 2-3 & 26-28 & \mathrm{n} / \mathrm{a} \\ 1 & 2-3 & 22 & \mathrm{n} / \mathrm{a} \\ 2 & 2-3 & 24 & \mathrm{p} \\ 2-3 & \mathrm{n} / \mathrm{a} & 20 & \mathrm{n} / \mathrm{a} \\ 2-4 & 2-3 & 24-28 & \mathrm{p}, \mathrm{a} \\ 2-3 & 2-3 & 20 & \mathrm{a} \\ 2 & 2-3 & 24 & \mathrm{p} \\ 2 & 2-3 & 20 & \mathrm{n} / \mathrm{a}\end{array}$

$\begin{array}{lllllclclll}\mathrm{d} & \mathrm{r} & \mathrm{nc} & \mathrm{a} & \mathrm{i} & \mathrm{s} 1,2 & \mathrm{a} & 2 & 2-3 & 18 & \mathrm{a} \\ \mathrm{d} & \mathrm{r} & \mathrm{nc} & \mathrm{a} & \mathrm{i} & 2 & \mathrm{a} & 1 & 2-3 & 20 & \mathrm{p} \\ \mathrm{d} & \mathrm{r} & \mathrm{nc} & \mathrm{a} & \mathrm{c} & 2 ? & \mathrm{a} & 2 & 2-3 & 20 & \mathrm{a} \\ \mathrm{d} & \mathrm{r} & \mathrm{nc} & \mathrm{a} & \mathrm{c} & 2 & \mathrm{a} & 0-1 ? & 2-3 & 20 & \mathrm{a} \\ \mathrm{i} & \mathrm{r} & \mathrm{nc} & \mathrm{a} & \mathrm{c} & 2 & \mathrm{p} & 2 & 2-3 & 18 & \mathrm{a} \\ \mathrm{d} & \mathrm{r} & \mathrm{nc} & \mathrm{a} & \mathrm{i} & 2 & \mathrm{a} & 1 & 2-3 & 16-18 & \mathrm{p} \\ \mathrm{i} & \mathrm{r} & \mathrm{nc} & \mathrm{a} & \mathrm{i} & 2 & \mathrm{a} & 1 & 2-3 & 18 & \mathrm{a} \\ \mathrm{d} & \mathrm{r} & \mathrm{nc} & \mathrm{a} & \mathrm{i} & 2 & \mathrm{a} & 1-4 ? & 2-3 & 20 & \mathrm{p} \\ \mathrm{i} & \mathrm{r} & \mathrm{nc} & \mathrm{a} & \mathrm{c} & 2 & \mathrm{n} / \mathrm{a} & \mathrm{n} / \mathrm{a} & 2-3 & 18 & \mathrm{a}\end{array}$


Amerotyphlops tasymicris Amerotyphlops tenuis Amerotyphlops tycherus Amerotyphlops yonenagae Antillotyphlops annae Antillotyphlops dominicanus Antillotyphlops geotomus Antillotyphlops granti Antillotyphlops guadeloupensis Antillotyphlops hypomethes Antillotyphlops monastus Antillotyphlops monensis Antillotyphlops naugus Antillotyphlops platycephalus Antillotyphlops richardi Cubatyphlops anchaurus Cubatyphlops anousius Cubatyphlops arator Cubatyphlops biminiensis Cubatyphlops caymanensis Cubatyphlops contorhinus Cubatyphlops epactius Cubatyphlops golyathi Cubatyphlops paradoxus Cubatyphlops perimychus Cubatyphlops satelles Typhlops agoralionis Typhlops capitulatus Typhlops eperopeus Typhlops gonavensis Typhlops hectus Typhlops jamaicensis Typhlops leptolepis Typhlops lumbricalis Typhlops oxyrhinus Typhlops pachyrhinus Typhlops proancylops Typhlops pusillus Typhlops rostellatus Typhlops schwartzi Typhlops silus Typhlops sulcatus Typhlops sylleptor Typhlops syntherus Typhlops tetrathyreus Typhlops titanops Amerotyphlops trinitatus Antillotyphlops catapontus Cubatyphlops notorachius

\begin{tabular}{|c|c|c|c|c|c|c|c|c|c|c|}
\hline$d$ & $r$ & $\mathrm{nc}$ & a & c & 1 & a & 1 & $2-3$ & 20 & $a$ \\
\hline i & $r$ & nc & $a$ & c & 2 & a & $2-3$ & $2-3$ & $17-18$ & $a$ \\
\hline$d$ & $r$ & $\mathrm{nc}$ & $a$ & c & s1 & a & 1 & $2-3$ & 20 & $a$ \\
\hline $\mathrm{i}$ & $r$ & $\mathrm{nc}$ & a & c & 2 & a & $3-4$ & $2-3$ & 22 & $\mathrm{a}$ \\
\hline$d$ & $r$ & $\mathrm{nc}$ & a & i & 2 & a & 2 & $2-3$ & 18 & $\mathrm{a}$ \\
\hline d & $r$ & $\mathrm{nc}$ & a & c & $\mathrm{n} / \mathrm{a}$ & $\mathrm{a}$ & 2 & $3-4 ?$ & $20-22$ & $p$ \\
\hline $\mathrm{n} / \mathrm{a}$ & $\mathrm{n} / \mathrm{a}$ & $\mathrm{nc}$ & a & c & 2 & $\mathrm{n} / \mathrm{a}$ & 2 & $\mathrm{n} / \mathrm{a}$ & 20 & $p$ \\
\hline$d$ & $r$ & $\mathrm{nc}$ & a & c & 2 & $\mathrm{a}$ & 2 & 3 & $22-24$ & $p$ \\
\hline$d$ & $r$ & $\mathrm{nc}$ & a & c & 2 & $\mathrm{n} / \mathrm{a}$ & 2 & 3 & $20-22$ & $p$ \\
\hline$d$ & $r$ & $\mathrm{nc}$ & a & c & 2 & $\mathrm{a}$ & 2 & 3 & 16 & $p$ \\
\hline$d$ & $r$ & $\mathrm{nc}$ & a & c & 2 & a & 2 & 3 & 24 & $p$ \\
\hline$d$ & $r$ & $\mathrm{nc}$ & $a$ & c & 2 & $a$ & $2(1)$ & 3 & 20 & $p$ \\
\hline$d$ & $r$ & $\mathrm{nc}$ & $a$ & c & 2 & $a$ & 2 & 3 & $20-22$ & $p$ \\
\hline$d$ & $\mathrm{a}$ & $\mathrm{nc}$ & $a$ & c & 2 & $a$ & 2 & 3 & 20 & $p$ \\
\hline $\mathrm{n} / \mathrm{a}$ & $\mathrm{n} / \mathrm{a}$ & $\mathrm{nc}$ & a & c & 2 & $\mathrm{n} / \mathrm{a}$ & 2 & $\mathrm{n} / \mathrm{a}$ & $18-20$ & $p$ \\
\hline$d$ & $r$ & $\mathrm{nc}$ & $a$ & c & 2 & $\mathrm{a}$ & $\mathrm{n} / \mathrm{a}$ & 3 & $20-22$ & $p$ \\
\hline$d$ & $r$ & $\mathrm{nc}$ & a & c & 2 & $a$ & 2 & 3 & $20-22$ & $p$ \\
\hline$d$ & $r$ & $\mathrm{nc}$ & a & c & $\mathrm{n} / \mathrm{a}$ & $a$ & 1 & $2-3$ & 22 & $p$ \\
\hline$d$ & $r$ & $\mathrm{nc}$ & a & c & $\mathrm{n} / \mathrm{a}$ & a & 1 & $2-3$ & 22 & $p$ \\
\hline$d$ & $r$ & $\mathrm{nc}$ & a & c & 2 & a & 1 & $2-3$ & 24 & $p$ \\
\hline$d$ & $r$ & $\mathrm{nc}$ & $a$ & c & 2 & a & $1(2)$ & $2-3$ & $22-24$ & $\mathrm{p}, \mathrm{a}$ \\
\hline$d$ & $r$ & $\mathrm{nc}$ & a & c & 2 & a & $1(2)$ & $2-3$ & 20 & $a$ \\
\hline$d$ & $r$ & $\mathrm{nc}$ & $a$ & c & 2 & $\mathrm{a}$ & 1 & $2-3$ & 22 & $p$ \\
\hline$d$ & $r$ & $\mathrm{nc}$ & a & c & $\mathrm{n} / \mathrm{a}$ & a & 1 & $2-3$ & 22 & $a(p)$ \\
\hline$d$ & $r$ & $\mathrm{nc}$ & $a$ & c & 2 & $\mathrm{a}$ & 1 & $2-3$ & 22 & $\mathrm{p}$ \\
\hline$d$ & $r$ & $\mathrm{nc}$ & a & c & $\mathrm{n} / \mathrm{a}$ & a & 1 & $2-3$ & 22 & $p$ \\
\hline$d$ & $r$ & $\mathrm{nc}$ & a & c & 2 & a & 1 & $2-3$ & 22 & $\mathrm{a}$ \\
\hline$d$ & $r$ & $\mathrm{nc}$ & a & c & $\mathrm{n} / \mathrm{a}$ & a & 1 & $2-3$ & 22 & $p, a$ \\
\hline$d$ & $r$ & $\mathrm{nc}$ & a & c & 2 & a & 1 & $2-3$ & 22 & $p$ \\
\hline$d$ & $r$ & $\mathrm{nc}$ & a & c & $\mathrm{n} / \mathrm{a}$ & a & 2 & 3 & 20 & $a$ \\
\hline$d$ & $r$ & $\mathrm{nc}$ & a & c & 2 & a & 2(1) & 3 & 20 & a \\
\hline$d$ & $r$ & $\mathrm{nc}$ & a & c & 2 & a & 2 & 3 & 20 & $p$ \\
\hline$d$ & $r$ & $\mathrm{nc}$ & a & c & 2 & a & 2 & 3 & 20 & $a$ \\
\hline$d$ & $r$ & $\mathrm{nc}$ & a & c & 2 & a & $2(3)$ & 3 & $20-22$ & $p, a$ \\
\hline$d$ & $r$ & $\mathrm{nc}$ & a & c & 2 & a & $2(1-3)$ & 3 & 22 & $a(p)$ \\
\hline$d$ & $r$ & $\mathrm{nc}$ & a & c & 2 & a & 2 & 3 & $18-20$ & $p$ \\
\hline$d$ & $r$ & $\mathrm{nc}$ & a & c & 2 & a & $2(1-3)$ & 3 & $18-20$ & $p$ \\
\hline$d$ & $r$ & $\mathrm{nc}$ & $\mathrm{a}$ & c & 2 & $\mathrm{a}$ & 2 & 3 & $18-20$ & $p$ \\
\hline$d$ & $r$ & $\mathrm{nc}$ & a & c & 2 & a & 2 & 3 & $18-20$ & $p$ \\
\hline$d$ & $r$ & $\mathrm{nc}$ & a & c & 2 & a & 2 & 3 & 20 & a \\
\hline$d$ & $r$ & $\mathrm{nc}$ & a & c & 2 & $p$ & $2(1-3)$ & Os & $20-22$ & $p, a$ \\
\hline$d$ & $r$ & $\mathrm{nc}$ & a & c & 2 & a & $2(1)$ & 3 & $18-20$ & $p$ \\
\hline$d$ & $r$ & $\mathrm{nc}$ & $a$ & c & 2 & $a$ & 1 & 3 & $18-20$ & $p$ \\
\hline$d$ & $\mathrm{n} / \mathrm{a}$ & $\mathrm{nc}$ & $a$ & $c, i$ & 2 & $a$ & 2 & 3 & $18-20$ & $p$ \\
\hline$d$ & $r$ & $\mathrm{nc}$ & a & c & 2 & a & 1 & 3 & 20 & $a(p)$ \\
\hline$d$ & $\mathrm{n} / \mathrm{a}$ & $\mathrm{nc}$ & $\mathrm{a}$ & c & 2 & a & 2 & 3 & 20 & $a$ \\
\hline$d$ & $r$ & $\mathrm{nc}$ & $a$ & c & 2 & a & $1(2)$ & 3 & 22 & a \\
\hline$d$ & $r$ & $\mathrm{nc}$ & a & c & 2 & a & 2 & 3 & $18-20$ & $p$ \\
\hline$d$ & $r$ & $\mathrm{nc}$ & a & c & 2 & $\mathrm{a}$ & 2 & 3 & 20 & $p$ \\
\hline
\end{tabular}

\begin{tabular}{lccccccccc}
\hline & $12 \mathrm{a}$ & $12 \mathrm{~b}$ & $13 \mathrm{a}$ & $13 \mathrm{~b}$ & $14 \mathrm{a}$ & $15 \mathrm{a}$ & $15 \mathrm{~b}$ & $16 \mathrm{a}$ & $16 \mathrm{~b}$ \\
\hline AFROTYPHLOPINAE & & & & & & & & & \\
Afrotyphlops angolensis & $350-578$ & 464 & $\mathrm{n} / \mathrm{a}$ & $\mathrm{n} / \mathrm{a}$ & 660 & $26-60$ & 43.0 & 58.8 & 58.8 \\
Afrotyphlops anomalus & $365-431$ & 398 & $\mathrm{n} / \mathrm{a}$ & $\mathrm{n} / \mathrm{a}$ & 540 & $29-37$ & 33.0 & 47 & 47.0 \\
Afrotyphlops bibronii & $363-453$ & 408 & $\mathrm{n} / \mathrm{a}$ & $\mathrm{n} / \mathrm{a}$ & 484 & $31-42$ & 36.5 & 70.5 & 70.5 \\
Afrotyphlops blanfordii & $343-424$ & 384 & $\mathrm{n} / \mathrm{a}$ & $\mathrm{n} / \mathrm{a}$ & 343 & $29-41$ & 35.0 & $\mathrm{n} / \mathrm{a}$ & $\mathrm{n} / \mathrm{a}$
\end{tabular}


Afrotyphlops brevis

Afrotyphlops calabresii

Afrotyphlops comorensis

Afrotyphlops congestus

Afrotyphlops cuneirostris

Afrotyphlops decorosus

Afrotyphlops elegans

Afrotyphlops fornasinii

Afrotyphlops gierrai

Afrotyphlops jubanus

Afrotyphlops kaimosae

Afrotyphlops liberiensis

Afrotyphlops lineolatus

Afrotyphlops mucruso

Afrotyphlops nanus

Afrotyphlops nigrocandidus

Afrotyphlops obtusus

Afrotyphlops platyrhynchus

Afrotyphlops punctatus

Afrotyphlops rondoensis

Afrotyphlops schlegelii

Afrotyphlops schmidti

Afrotyphlops steinhausi

Afrotyphlops tanganicanus

Afrotyphlops usambaricus

Letheobia acutirostrata

Letheobia angeli

Letheobia caeca

Letheobia coecata

Letheobia crossii

Letheobia debilis

Letheobia episcopa

Letheobia erythraea

Letheobia feae

Letheobia gracilis

Letheobia graueri

Letheobia kibarae

Letheobia largeni

Letheobia leucosticta

Letheobia lumbriciformis

Letheobia manni

Letheobia newtoni

Letheobia pallida

Letheobia pauwelsi

Letheobia pembana

Letheobia praeocularis

Letheobia rufescens

Letheobia simonii

Letheobia somalica

Letheobia stejnegeri

Letheobia sudanensis

Letheobia swahilica

Letheobia toritensis

Letheobia uluguruensis

Letheobia wittei

Letheobia zenkeri

Rhinotyphlops ataeniatus

\begin{tabular}{|c|c|c|c|c|c|c|c|c|}
\hline $288-557$ & 423 & $\mathrm{n} / \mathrm{a}$ & $n / a$ & 765 & $17-47$ & 32.0 & $\mathrm{n} / \mathrm{a}$ & $\mathrm{n} / \mathrm{a}$ \\
\hline $257-302$ & 280 & $8-12$ & 10 & 189 & $18-37$ & 27.5 & $\mathrm{n} / \mathrm{a}$ & $\mathrm{n} / \mathrm{a}$ \\
\hline 384-485 & 435 & $12-15$ & 13.5 & 245 & $42-61$ & 51.5 & $33.3-50$ & 41.7 \\
\hline $310-416$ & 363 & 10 & 10 & 765 & $19-29$ & 24.0 & 44.3 & 44.3 \\
\hline $216-257$ & 237 & $\mathrm{n} / \mathrm{a}$ & $\mathrm{n} / \mathrm{a}$ & 158 & $21-40$ & 30.5 & 58.4 & 58. \\
\hline $460-542$ & 501 & $\mathrm{n} / \mathrm{a}$ & $\mathrm{n} / \mathrm{a}$ & 510 & $45-66$ & 55.5 & 94.3 & 94.3 \\
\hline $315-349$ & 332 & $\mathrm{n} / \mathrm{a}$ & $\mathrm{n} / \mathrm{a}$ & 420 & $38.2-57.4$ & 47.8 & 52 & 52.0 \\
\hline $232-286$ & 259 & $\mathrm{n} / \mathrm{a}$ & $\mathrm{n} / \mathrm{a}$ & 185 & 28-45 & 36.5 & 37.1 & 37.1 \\
\hline $372-463$ & 418 & $\mathrm{n} / \mathrm{a}$ & $\mathrm{n} / \mathrm{a}$ & 469 & $32-58$ & 45.0 & $\mathrm{n} / \mathrm{a}$ & $\mathrm{n} /$ \\
\hline $391-430$ & 411 & $\mathrm{n} / \mathrm{a}$ & $\mathrm{n} / \mathrm{a}$ & 510 & $44-57$ & 50.5 & $\mathrm{n} / \mathrm{a}$ & $\mathrm{n} / \mathrm{a}$ \\
\hline 390 & 390 & $\mathrm{n} / \mathrm{a}$ & $\mathrm{n} / \mathrm{a}$ & 215 & 43 & 43.0 & 53.8 & 53.8 \\
\hline $\mathrm{n} / \mathrm{a}$ & $\mathrm{n} / \mathrm{a}$ & $\mathrm{n} / \mathrm{a}$ & $\mathrm{n} / \mathrm{a}$ & $\mathrm{n} / \mathrm{a}$ & $\mathrm{n} / \mathrm{a}$ & $\mathrm{n} / \mathrm{a}$ & $\mathrm{n} / \mathrm{a}$ & $n / a$ \\
\hline $323-505$ & 414 & $\mathrm{n} / \mathrm{a}$ & $\mathrm{n} / \mathrm{a}$ & 640 & $21-47$ & 34.0 & 40 & 40.0 \\
\hline $307-517$ & 412 & $\mathrm{n} / \mathrm{a}$ & $\mathrm{n} / \mathrm{a}$ & 950 & $21-58$ & 39.5 & 49-79 & 64.0 \\
\hline $284-290$ & 287 & $10-11$ & 10.5 & 125 & $23-27$ & 25.0 & $41.7-47.6$ & 44.7 \\
\hline $464-542$ & 503 & $\mathrm{n} / \mathrm{a}$ & $\mathrm{n} / \mathrm{a}$ & 573 & $38-51$ & 44.5 & 62.9 & 62.9 \\
\hline $406-507$ & 457 & 4 & 4 & 380 & $46-95$ & 70.5 & 151.5 & 151.5 \\
\hline $400-425$ & 413 & $\mathrm{n} / \mathrm{a}$ & $\mathrm{n} / \mathrm{a}$ & 273 & $50-60$ & 55.0 & $\mathrm{n} / \mathrm{a}$ & $\mathrm{n} / \mathrm{a}$ \\
\hline $374-465$ & 420 & $\mathrm{n} / \mathrm{a}$ & $\mathrm{n} / \mathrm{a}$ & 660 & $26-33$ & 29.5 & $\mathrm{n} / \mathrm{a}$ & $\mathrm{n} / \mathrm{a}$ \\
\hline $312-379$ & 346 & $\mathrm{n} / \mathrm{a}$ & $\mathrm{n} / \mathrm{a}$ & 370 & $33-45$ & 39.0 & $57-78$ & 67.5 \\
\hline $341-620$ & 481 & $\mathrm{n} / \mathrm{a}$ & $\mathrm{n} / \mathrm{a}$ & 804 & $19-46$ & 32.5 & 45.5 & 45.5 \\
\hline $317-374$ & 346 & $\mathrm{n} / \mathrm{a}$ & $\mathrm{n} / \mathrm{a}$ & 605 & $22-49$ & 35.5 & $\mathrm{n} / \mathrm{a}$ & $\mathrm{n} / \mathrm{a}$ \\
\hline $378-430$ & 404 & $\mathrm{n} / \mathrm{a}$ & $\mathrm{n} / \mathrm{a}$ & 620 & $41-45$ & 43.0 & $n / a$ & $\mathrm{n} /$ \\
\hline $352-425$ & 389 & $\mathrm{n} / \mathrm{a}$ & $\mathrm{n} / \mathrm{a}$ & 390 & $29-47$ & 38.0 & $n / a$ & $n / a$ \\
\hline $344-389$ & 367 & $\mathrm{n} / \mathrm{a}$ & $\mathrm{n} / \mathrm{a}$ & 605 & $27-31$ & 29.0 & $n / a$ & $\mathrm{n} /$ \\
\hline $\mathrm{n} / \mathrm{a}$ & $\mathrm{n} / \mathrm{a}$ & $\mathrm{n} / \mathrm{a}$ & $\mathrm{n} / \mathrm{a}$ & $n / a$ & $\mathrm{n} / \mathrm{a}$ & $\mathrm{n} / \mathrm{a}$ & $\mathrm{n} / \mathrm{a}$ & $\mathrm{n} / \mathrm{a}$ \\
\hline$n / a$ & $\mathrm{n} / \mathrm{a}$ & $\mathrm{n} / \mathrm{a}$ & $\mathrm{n} / \mathrm{a}$ & 332 & $\mathrm{n} / \mathrm{a}$ & $n / a$ & $n / a$ & $\mathrm{n} /$ \\
\hline $417-561$ & 489 & $\mathrm{n} / \mathrm{a}$ & $\mathrm{n} / \mathrm{a}$ & 425 & $\mathrm{n} / \mathrm{a}$ & $\mathrm{n} / \mathrm{a}$ & 76 & 76.0 \\
\hline $282-334$ & 308 & $\mathrm{n} / \mathrm{a}$ & $\mathrm{n} / \mathrm{a}$ & 165 & $\mathrm{n} / \mathrm{a}$ & $\mathrm{n} / \mathrm{a}$ & 73.5 & 73.5 \\
\hline $459-513$ & 486 & $8-13$ & 10.5 & 310 & $54-85$ & 69.5 & $55.6-100$ & 77.8 \\
\hline $547-668$ & 608 & 11 & 11 & 480 & 98-129 & 113.5 & $125.8-135.7$ & 130 \\
\hline $544-581$ & 563 & $11-15$ & 13 & 318 & $74-90$ & 82.0 & 61.4-111.1 & 86.3 \\
\hline $443-462$ & 453 & 11 & 11 & 250 & $68-82$ & 75.0 & 58.8 & 58.8 \\
\hline $407-480$ & 444 & 8 & 8 & 330 & $51-68$ & 59.5 & 66.7 & 66.7 \\
\hline $608-737$ & 673 & $\mathrm{n} / \mathrm{a}$ & $\mathrm{n} / \mathrm{a}$ & 550 & 70-107 & 88.5 & $\mathrm{n} / \mathrm{a}$ & $\mathrm{n} / \mathrm{a}$ \\
\hline $454-622$ & 538 & $\mathrm{n} / \mathrm{a}$ & $\mathrm{n} / \mathrm{a}$ & 450 & $60-89$ & 74.5 & $\mathrm{n} / \mathrm{a}$ & $\mathrm{n} / \mathrm{a}$ \\
\hline $562-623$ & 593 & $\mathrm{n} / \mathrm{a}$ & $\mathrm{n} / \mathrm{a}$ & 525 & $58-87$ & 72.5 & $\mathrm{n} / \mathrm{a}$ & $\mathrm{n} / \mathrm{a}$ \\
\hline 432 & 432 & $\mathrm{n} / \mathrm{a}$ & $\mathrm{n} / \mathrm{a}$ & 274 & $64-65$ & 64.5 & $\mathrm{n} / \mathrm{a}$ & $\mathrm{n} / \mathrm{a}$ \\
\hline 336 & 336 & $\mathrm{n} / \mathrm{a}$ & $\mathrm{n} / \mathrm{a}$ & 230 & $45-47$ & 46.0 & $n / a$ & $\mathrm{n} / \mathrm{a}$ \\
\hline $465-607$ & 536 & $\mathrm{n} / \mathrm{a}$ & $\mathrm{n} / \mathrm{a}$ & 450 & $38-83$ & 60.5 & $\mathrm{n} / \mathrm{a}$ & $\mathrm{n} / \mathrm{a}$ \\
\hline $480-508$ & 494 & $\mathrm{n} / \mathrm{a}$ & $\mathrm{n} / \mathrm{a}$ & 360 & 40 & 40.0 & 68.6 & 68.6 \\
\hline $446-557$ & 502 & 12 & 12 & 400 & $50-70$ & 60.0 & $52.6-71.4$ & 62.0 \\
\hline $418-429$ & 424 & $\mathrm{n} / \mathrm{a}$ & $\mathrm{n} / \mathrm{a}$ & 265 & $53-62$ & 57.5 & $\mathrm{n} / \mathrm{a}$ & $\mathrm{n} / \mathrm{a}$ \\
\hline 483 & 483 & 11 & 11 & 310 & 81.6 & 81.6 & 62.5 & 62.5 \\
\hline 353 & 353 & $\mathrm{n} / \mathrm{a}$ & $\mathrm{n} / \mathrm{a}$ & 158 & 53 & 53.0 & $n / a$ & $n / a$ \\
\hline $493-544$ & 519 & $\mathrm{n} / \mathrm{a}$ & $\mathrm{n} / \mathrm{a}$ & 340 & 67 & 67.0 & $n / a$ & $\mathrm{n} / \mathrm{a}$ \\
\hline $585-656$ & 621 & $\mathrm{n} / \mathrm{a}$ & $\mathrm{n} / \mathrm{a}$ & 520 & $69-90$ & 79.5 & $\mathrm{n} / \mathrm{a}$ & $\mathrm{n} / \mathrm{a}$ \\
\hline $403-488$ & 446 & 9-14 & 11.5 & 239 & 49-91 & 70.0 & $45.5-100$ & 72.8 \\
\hline $555-656$ & 606 & $11-13$ & 12 & 670 & $44-90$ & 67.0 & 71.4-142.9 & 107. \\
\hline $488-548$ & 518 & $\mathrm{n} / \mathrm{a}$ & $\mathrm{n} / \mathrm{a}$ & 465 & $46-53$ & 49.5 & 83.6 & 83. \\
\hline $569-660$ & 615 & $n / a$ & $\mathrm{n} / \mathrm{a}$ & 520 & $57-95$ & 76.0 & $\mathrm{n} / \mathrm{a}$ & $\mathrm{n} / \mathrm{a}$ \\
\hline $376-392$ & 384 & $\mathrm{n} / \mathrm{a}$ & $\mathrm{n} / \mathrm{a}$ & 190 & $49-62$ & 55.5 & $\mathrm{n} / \mathrm{a}$ & $\mathrm{n} / \mathrm{a}$ \\
\hline $427-487$ & 457 & $\mathrm{n} / \mathrm{a}$ & $\mathrm{n} / \mathrm{a}$ & 268 & $62-90$ & 76.0 & $\mathrm{n} / \mathrm{a}$ & $\mathrm{n} / \mathrm{a}$ \\
\hline $379-416$ & 398 & $\mathrm{n} / \mathrm{a}$ & $\mathrm{n} / \mathrm{a}$ & 245 & $48-57$ & 52.5 & $\mathrm{n} / \mathrm{a}$ & $n / a$ \\
\hline $501-511$ & 506 & $n / a$ & $\mathrm{n} / \mathrm{a}$ & 310 & 68 & 68.0 & $\mathrm{n} / \mathrm{a}$ & \\
\hline $250-281$ & 266 & $\mathrm{n} / \mathrm{a}$ & $\mathrm{n} / \mathrm{a}$ & 135 & $35-45$ & 40.0 & $\mathrm{n} / \mathrm{a}$ & $\mathrm{n} / \mathrm{s}$ \\
\hline $443-531$ & 487 & $\mathrm{n} / \mathrm{a}$ & $\mathrm{n} / \mathrm{a}$ & 455 & $34-72$ & 53.0 & $\mathrm{n} / \mathrm{a}$ & \\
\hline
\end{tabular}


Rhinotyphlops boylei

Rhinotyphlops lalandei

Rhinotyphlops leucocephalus

Rhinotyphlops schinzi

Rhinotyphlops scortecci

Rhinotyphlops unitaeniatus

\section{ASIATYPHLOPINAE}

Acutotyphlops banaorum

Acutotyphlops infralabialis

Acutotyphlops kunuaensis

Acutotyphlops solomonis

Acutotyphlops subocularis

Anilios affinis

Anilios ammodytes

Anilios aspinus

Anilios australis

Anilios batillus

Anilios bicolor

Anilios bituberculatus

Anilios broomi

Anilios centralis

Anilios chamodracaena

Anilios diversus

Anilios endoterus

Anilios erycinus

Anilios ganei

Anilios grypus

Anilios guentheri

Anilios hamatus

Anilios howi

Anilios kimberleyensis

Anilios leptosomus

Anilios leucoproctus

Anilios ligatus

Anilios longissimus

Anilios margaretae

Anilios micrommus

Anilios minimus

Anilios nema

Anilios nigrescens

Anilios nigroterminatus

Anilios pilbarensis

Anilios pinguis

Anilios proximus

Anilios robertsi

Anilios silvia

Anilios splendidus

Anilios torresianus

Anilios tovelli

Anilios troglodytes

Anilios unguirostris

Anilios waitii

Anilios wiedii

Anilios yampiensis

Anilios yirrikalae

Asiatyphlops bothriorhynchus

Asiatyphlops diardii

\begin{tabular}{|c|c|c|c|c|c|c|c|c|}
\hline 344-379 & 362 & $\mathrm{n} / \mathrm{a}$ & $\mathrm{n} / \mathrm{a}$ & 223 & $33-53$ & 43.0 & 60.7 & 60.7 \\
\hline 334-467 & 401 & $\mathrm{n} / \mathrm{a}$ & $\mathrm{n} / \mathrm{a}$ & 355 & $31-63$ & 47.0 & $\mathrm{n} / \mathrm{a}$ & $\mathrm{n} / \mathrm{a}$ \\
\hline 369 & 369 & $\mathrm{n} / \mathrm{a}$ & $\mathrm{n} / \mathrm{a}$ & 220 & 43 & 43.0 & 70.7 & 70.7 \\
\hline $413-538$ & 476 & $14-15$ & 14.5 & 293 & $34-64$ & 49.0 & 50.2 & 50.2 \\
\hline $311-405$ & 358 & $\mathrm{n} / \mathrm{a}$ & $\mathrm{n} / \mathrm{a}$ & 280 & $27-73$ & 50.0 & $\mathrm{n} / \mathrm{a}$ & $\mathrm{n} / \mathrm{a}$ \\
\hline 467-586 & 527 & $\mathrm{n} / \mathrm{a}$ & $\mathrm{n} / \mathrm{a}$ & 435 & $38-77$ & 57.5 & 78.5 & 78.5 \\
\hline $352-361$ & 357 & $16-19$ & 17.5 & 333 & $32.1-51.2$ & 41.7 & $25-27.8$ & \\
\hline $418-526$ & 472 & $14-28$ & 21 & 372 & $33.4-57.4$ & 45.4 & $18.9-100$ & 59.5 \\
\hline $360-542$ & 451 & $11-31$ & 21 & 373 & $22.4-57.6$ & 40.0 & 21.1 & 21. \\
\hline 334-424 & 379 & $18-30$ & 24 & 487 & $18.2-42.8$ & 30.5 & $13-34.5$ & 23.8 \\
\hline $363-472$ & 418 & $14-28$ & 21 & 394 & $23.2-43.8$ & 33.5 & $15.9-33.3$ & 24.6 \\
\hline 278-357 & 318 & $\mathrm{n} / \mathrm{a}$ & $\mathrm{n} / \mathrm{a}$ & 220 & $45-60$ & 52.5 & n/a & $n / a$ \\
\hline $397-432$ & 415 & $10-16$ & 13 & 252 & 57.5 & 57.5 & $19.6-62.5$ & \\
\hline $413-444$ & 429 & $10-16$ & 13 & 281 & $63.6-75.8$ & 69.7 & 42.8 & 42.8 \\
\hline $305-401$ & 353 & $10-18$ & 14 & 500 & $24-50$ & 37.0 & $22-41.7$ & 31.9 \\
\hline 557 & 557 & $\mathrm{n} / \mathrm{a}$ & $\mathrm{n} / \mathrm{a}$ & 320 & 53 & 53.0 & 45.7 & 45.7 \\
\hline $300-380$ & 340 & 12 & 12 & 275 & 34.4 & 34.4 & $22-45.8$ & 33.9 \\
\hline $425-503$ & 464 & $11-18$ & 14.5 & 450 & $40-90$ & 65.0 & $30.3-66.7$ & 48.5 \\
\hline $456-460$ & 458 & $15-16$ & 15.5 & 250 & $30-60$ & 45.0 & $\mathrm{n} / \mathrm{a}$ & $n / a$ \\
\hline $417-502$ & 460 & $12-20$ & 16 & 306 & $60-69$ & 64.5 & $32.3-66.7$ & 49.5 \\
\hline $478-539$ & 509 & $14-16$ & 15 & 210 & $\mathrm{n} / \mathrm{a}$ & $\mathrm{n} / \mathrm{a}$ & $33.3-100$ & 66.7 \\
\hline $397-475$ & 436 & 8-18 & 13 & 352 & $40-70$ & 55.0 & $28.6-71.4$ & 50. \\
\hline $415-454$ & 435 & $9-16$ & 12.5 & 400 & $40-60$ & 50.0 & $34.5-66.7$ & 50.6 \\
\hline $315-335$ & 325 & $16-23$ & 19.5 & 350 & $27.1-44$ & 35.6 & 35 & 35.0 \\
\hline $430-448$ & 439 & $12-19$ & 15.5 & 340 & $36.2-46.9$ & 41.6 & $33.9-64.2$ & 49.1 \\
\hline $538-713$ & 626 & $13-36$ & 24.5 & 450 & $63-122$ & 92.5 & $22.7-90.9$ & 56.8 \\
\hline $535-595$ & 565 & $10-15$ & 12.5 & 400 & $40-90$ & 65.0 & 54.6 & 54.6 \\
\hline $330-396$ & 363 & $12-18$ & 15 & 386 & $28.7-58.7$ & 43.7 & $24.4-58.8$ & 41.6 \\
\hline 450 & 450 & 16 & 16 & 210 & $\mathrm{n} / \mathrm{a}$ & $\mathrm{n} / \mathrm{a}$ & 34.5 & 34.5 \\
\hline $500-524$ & 512 & $12-20$ & 16 & 296 & $\mathrm{n} / \mathrm{a}$ & $\mathrm{n} / \mathrm{a}$ & $45.5-58.8$ & 52.2 \\
\hline $660-665$ & 663 & $16-25$ & 20.5 & 400 & $70-88$ & 79.0 & $26.3-66.7$ & 46.5 \\
\hline $386-426$ & 406 & $14-17$ & 15.5 & 250 & $40-65$ & 52.5 & $42.2-51.3$ & 46.8 \\
\hline $346-452$ & 399 & $11-17$ & 14 & 500 & $20-40$ & 30.0 & $18.1-37$ & 27.6 \\
\hline 750 & 750 & 15 & 15 & 268 & 134.2 & 134.2 & 111.8 & 111 \\
\hline $571-571$ & 571 & 12 & 12 & 306 & $\mathrm{n} / \mathrm{a}$ & $\mathrm{n} / \mathrm{a}$ & 90.9 & 90.9 \\
\hline $493-493$ & 493 & 15 & 15 & 205 & $\mathrm{n} / \mathrm{a}$ & $\mathrm{n} / \mathrm{a}$ & 41.7 & 41.7 \\
\hline 457 & 457 & $9-17$ & 13 & 200 & $51.5-70.4$ & 61.0 & $\mathrm{n} / \mathrm{a}$ & $\mathrm{n} / \mathrm{a}$ \\
\hline 529-603 & 566 & 9-14 & 11.5 & 268 & $57.1-92.6$ & 74.9 & $\mathrm{n} / \mathrm{a}$ & $\mathrm{n} / \mathrm{a}$ \\
\hline $\mathrm{n} / \mathrm{a}$ & $\mathrm{n} / \mathrm{a}$ & $\mathrm{n} / \mathrm{a}$ & $\mathrm{n} / \mathrm{a}$ & 750 & $30-60$ & 45.0 & 45 & 45.0 \\
\hline $\mathrm{n} / \mathrm{a}$ & $\mathrm{n} / \mathrm{a}$ & $\mathrm{n} / \mathrm{a}$ & $\mathrm{n} / \mathrm{a}$ & 275 & 55-102 & 78.5 & 54 & 54.0 \\
\hline $363-425$ & 394 & $15-22$ & 18.5 & 371 & $42.3-57.9$ & 50.1 & $26.3-42.2$ & 34.3 \\
\hline $289-350$ & 320 & $12-19$ & 15.5 & 550 & $20-30$ & 25.0 & $17.5-38.5$ & 28.0 \\
\hline $\mathrm{n} / \mathrm{a}$ & $\mathrm{n} / \mathrm{a}$ & $\mathrm{n} / \mathrm{a}$ & $\mathrm{n} / \mathrm{a}$ & 750 & $20-40$ & 30.0 & 50.6 & 50.6 \\
\hline 568 & 568 & 12 & 12 & 290 & 67.4 & 67.4 & 61.6 & 61.6 \\
\hline $286-341$ & 314 & $17-21$ & 19 & 175 & $\mathrm{n} / \mathrm{a}$ & $\mathrm{n} / \mathrm{a}$ & $20-50$ & 35.0 \\
\hline 377 & 377 & 13 & 13 & 509 & 46.3 & 46.3 & 46.3 & 46.3 \\
\hline 365 & 365 & 19 & 19 & 400 & $34-43$ & 38.5 & $15-51.5$ & 33.3 \\
\hline $\mathrm{n} / \mathrm{a}$ & $\mathrm{n} / \mathrm{a}$ & $\mathrm{n} / \mathrm{a}$ & $\mathrm{n} / \mathrm{a}$ & 122 & $36-40$ & 38.0 & $29.2-34.9$ & 32.1 \\
\hline $655-655$ & 655 & 14 & 14 & 402 & $\mathrm{n} / \mathrm{a}$ & $\mathrm{n} / \mathrm{a}$ & 76.9 & 76.5 \\
\hline $279-506$ & 393 & $11-16$ & 13.5 & 700 & $38-70$ & 54.0 & $37-83.3$ & 60.2 \\
\hline $548-693$ & 621 & $13-26$ & 19.5 & 614 & $57-80$ & 68.5 & $32.3-100$ & 66.2 \\
\hline $\mathrm{n} / \mathrm{a}$ & $\mathrm{n} / \mathrm{a}$ & 10 & 10 & 300 & $30-80$ & 55.0 & 61.3 & 61.3 \\
\hline 491 & 491 & 11 & 11 & 128 & n/a & $\mathrm{n} / \mathrm{a}$ & 55.6 & 55. \\
\hline $\mathrm{n} / \mathrm{a}$ & $\mathrm{n} / \mathrm{a}$ & $\mathrm{n} / \mathrm{a}$ & $\mathrm{n} / \mathrm{a}$ & 200 & $60-70$ & 65.0 & $\mathrm{n} / \mathrm{a}$ & $\mathrm{n} / \mathrm{a}$ \\
\hline $300-330$ & 315 & 10 & 10 & 180 & 30 & 30.0 & $\mathrm{n} / \mathrm{a}$ & $\mathrm{n} /$ \\
\hline $260-303$ & 282 & $7-8$ & 7.5 & 430 & 30.2 & 30.2 & 73 & \\
\hline
\end{tabular}


Asiatyphlops fuscus

Asiatyphlops giadinhensis

Asiatyphlops klemmeri

Asiatyphlops koshunensis

Asiatyphlops leucomelas

Asiatyphlops muelleri

Asiatyphlops oatesii

Asiatyphlops roxaneae

Asiatyphlops siamensis

Asiatyphlops tenuicollis

Asiatyphlops trangensis

Cyclotyphlops deharvengi

Grypotyphlops acutus

Indotyphlops ahsanai

Indotyphlops albiceps

Indotyphlops braminus

Indotyphlops exiguus

Indotyphlops filiformis

Indotyphlops fletcheri

Indotyphlops hypsobothrius

Indotyphlops jerdoni

Indotyphlops khoratensis

Indotyphlops lankaensis

Indotyphlops lazelli

Indotyphlops loveridgei

Indotyphlops madgemintonae

Indotyphlops malcolmi

Indotyphlops meszoelyi

Indotyphlops ozakiae

Indotyphlops pammeces

Indotyphlops porrectus

Indotyphlops schmutzi

Indotyphlops tenebrarum

Indotyphlops veddae

Indotyphlops violaceus

Malayotyphlops canlaonensis

Malayotyphlops castanotus

Malayotyphlops collaris

Malayotyphlops hypogius

Malayotyphlops koekkoeki

Malayotyphlops kraali

Malayotyphlops luzonensis

Malayotyphlops manilae

Malayotyphlops ruber

Malayotyphlops ruficauda

Ramphotyphlops acuticauda

Ramphotyphlops adocetus

Ramphotyphlops angusticeps

Ramphotyphlops becki

Ramphotyphlops conradi

Ramphotyphlops cumingii

Ramphotyphlops depressus

Ramphotyphlops exocoeti

Ramphotyphlops flaviventer

Ramphotyphlops hatmaliyeb

Ramphotyphlops lineatus

Ramphotyphlops lorenzi

Ramphotyphlops mansuetus

\begin{tabular}{|c|c|c|c|c|c|c|c|}
\hline $\mathrm{n} / \mathrm{a}$ & $\mathrm{n} / \mathrm{a}$ & $\mathrm{n} / \mathrm{a}$ & $\mathrm{n} / \mathrm{a}$ & 214 & $\mathrm{n} / \mathrm{a}$ & $\mathrm{n} / \mathrm{a}$ & $42-42.8$ \\
\hline $316-340$ & 328 & $7-10$ & 8.5 & 238 & 26 & 26.0 & $43.5-47.6$ \\
\hline $291-292$ & 292 & $\mathrm{n} / \mathrm{a}$ & $\mathrm{n} / \mathrm{a}$ & 151 & 28 & 28.0 & 60.4 \\
\hline 246 & 246 & 26 & 26 & 290 & $39-48$ & 43.5 & $41.7-100$ \\
\hline $260-325$ & 293 & $\mathrm{n} / \mathrm{a}$ & $\mathrm{n} / \mathrm{a}$ & 130 & 32 & 32.0 & $\mathrm{n} / \mathrm{a}$ \\
\hline $384-439$ & 412 & $8-13$ & 10.5 & 355 & 32 & 32.0 & 88.8 \\
\hline $\mathrm{n} / \mathrm{a}$ & $\mathrm{n} / \mathrm{a}$ & $\mathrm{n} / \mathrm{a}$ & $\mathrm{n} / \mathrm{a}$ & 203 & $31-33$ & 32.0 & $\mathrm{n} / \mathrm{a}$ \\
\hline 329 & 329 & 5 & 5 & 231 & 38.5 & 38.5 & 76.9 \\
\hline 368 & 368 & 9 & 9 & 216 & 33 & 33.0 & $\mathrm{n} / \mathrm{a}$ \\
\hline $480-520$ & 500 & 12 & 12 & 365 & $64-70$ & 67.0 & 77.7 \\
\hline 370 & 370 & 11 & 11 & 155 & 30 & 30.0 & 55 \\
\hline 294 & 294 & $\mathrm{n} / \mathrm{a}$ & $\mathrm{n} / \mathrm{a}$ & 146 & 35.1 & 35.1 & 32.3 \\
\hline $448-526$ & 487 & $7-13$ & 10 & 630 & $30-66$ & 48.0 & $16.9-133.3$ \\
\hline 341 & 341 & 7 & 7 & 170 & 68 & 68.0 & 68 \\
\hline 317 & 317 & 12 & 12 & 194 & $39-64$ & 51.5 & $\mathrm{n} / \mathrm{a}$ \\
\hline $261-368$ & 315 & $8-15$ & 11.5 & 203 & $30-66$ & 48.0 & $34.5-50$ \\
\hline 348 & 348 & 12 & 12 & 196 & $60-78$ & 69.0 & $33.8-66.7$ \\
\hline $380-389$ & 385 & 8 & 8 & 135 & 60 & 60.0 & 58.7 \\
\hline $\mathrm{n} / \mathrm{a}$ & $\mathrm{n} / \mathrm{a}$ & $\mathrm{n} / \mathrm{a}$ & $\mathrm{n} / \mathrm{a}$ & 149 & $40-45$ & 42.5 & $\mathrm{n} / \mathrm{a}$ \\
\hline $\mathrm{n} / \mathrm{a}$ & $\mathrm{n} / \mathrm{a}$ & $\mathrm{n} / \mathrm{a}$ & $\mathrm{n} / \mathrm{a}$ & 285 & $52-71$ & 61.5 & $\mathrm{n} / \mathrm{a}$ \\
\hline $260-313$ & 287 & $\mathrm{n} / \mathrm{a}$ & $\mathrm{n} / \mathrm{a}$ & 280 & $35-46$ & 40.5 & 30 \\
\hline $315-331$ & 323 & $\mathrm{n} / \mathrm{a}$ & $\mathrm{n} / \mathrm{a}$ & 128 & $28-43.7$ & 35.9 & $34.1-53.5$ \\
\hline $229-261$ & 245 & $11-15$ & 13 & 130 & $29.1-33.3$ & 31.2 & $22.3-39.7$ \\
\hline $409-427$ & 418 & 10 & 10 & 158 & $77-83.2$ & 80.1 & 55.6 \\
\hline 430 & 430 & 11 & 11 & 208 & 83 & 83.0 & $52.6-59.4$ \\
\hline $336-364$ & 350 & $8-10$ & 9 & 200 & $62-130$ & 96.0 & $43-76$ \\
\hline $262-282$ & 272 & $9-11$ & 10 & 107 & 31 & 31.0 & $23.8-36.8$ \\
\hline $414-421$ & 418 & 9-10 & 9.5 & 179 & $53.8-71.4$ & 62.6 & $45.5-50$ \\
\hline $\mathrm{n} / \mathrm{a}$ & $\mathrm{n} / \mathrm{a}$ & $\mathrm{n} / \mathrm{a}$ & $\mathrm{n} / \mathrm{a}$ & $\mathrm{n} / \mathrm{a}$ & $\mathrm{n} / \mathrm{a}$ & $\mathrm{n} / \mathrm{a}$ & 34.8 \\
\hline $366-400$ & 383 & 11 & 11 & 140 & $55-75$ & 65.0 & $\mathrm{n} / \mathrm{a}$ \\
\hline $388-468$ & 428 & $7-12$ & 9.5 & 285 & $50-90$ & 70.0 & $43.5-71.4$ \\
\hline $403-413$ & 408 & $9-12$ & 10.5 & 140 & $62.5-93$ & 77.8 & $\mathrm{n} / \mathrm{a}$ \\
\hline $298-326$ & 312 & $12-14$ & 13 & 112 & $43.3-44.8$ & 44.1 & $36.1-55.5$ \\
\hline 295-309 & 302 & $13-14$ & 13.5 & 91 & 60 & 60.0 & 32.9 \\
\hline $245-308$ & 277 & 13 & 13 & 111 & 31 & 31.0 & 44.4 \\
\hline $\mathrm{n} / \mathrm{a}$ & $\mathrm{n} / \mathrm{a}$ & $\mathrm{n} / \mathrm{a}$ & $\mathrm{n} / \mathrm{a}$ & 122 & 34.1 & 34.1 & 48.8 \\
\hline $300-339$ & 320 & $10-14$ & 12 & 253 & 32 & 32.0 & $27.8-50$ \\
\hline $412-461$ & 437 & $9-13$ & 11 & 255 & 41 & 41.0 & $50-71.4$ \\
\hline 323 & 323 & 10 & 10 & 176 & 39.1 & 39.1 & 44 \\
\hline $\mathrm{n} / \mathrm{a}$ & $\mathrm{n} / \mathrm{a}$ & $\mathrm{n} / \mathrm{a}$ & $\mathrm{n} / \mathrm{a}$ & 445 & 40.4 & 40.4 & 62.6 \\
\hline $337-386$ & 362 & 12 & 12 & 295 & $38.5-42$ & 40.3 & $\mathrm{n} / \mathrm{a}$ \\
\hline 348 & 348 & 10 & 10 & 260 & 58 & 58.0 & $\mathrm{n} / \mathrm{a}$ \\
\hline $\mathrm{n} / \mathrm{a}$ & $\mathrm{n} / \mathrm{a}$ & 12 & 12 & 280 & 56 & 56.0 & 56 \\
\hline 360 & 360 & $<15$ & 14 & 225 & $36-37$ & 36.5 & $\mathrm{n} / \mathrm{a}$ \\
\hline 420 & 420 & $<15$ & 14 & 250 & $31-55$ & 43.0 & $\mathrm{n} / \mathrm{a}$ \\
\hline $\mathrm{n} / \mathrm{a}$ & $\mathrm{n} / \mathrm{a}$ & $\mathrm{n} / \mathrm{a}$ & $\mathrm{n} / \mathrm{a}$ & 123 & 52 & 52.0 & 30.8 \\
\hline $447-474$ & 461 & 32 & 32 & 390 & 38.1 & 38.1 & $18.3-30.3$ \\
\hline $617-653$ & 635 & $23-27$ & 25 & 455 & $65-87.5$ & 76.3 & 35 \\
\hline $206-241$ & 224 & $8-15$ & 11.5 & 117 & 24.3 & 24.3 & 23.4 \\
\hline 398 & 398 & 11 & 11 & 175 & $58.3-59.8$ & 59.1 & 70 \\
\hline $\mathrm{n} / \mathrm{a}$ & $\mathrm{n} / \mathrm{a}$ & $\mathrm{n} / \mathrm{a}$ & $\mathrm{n} / \mathrm{a}$ & 365 & $48-52$ & 50.0 & $\mathrm{n} / \mathrm{a}$ \\
\hline $289-438$ & 364 & $13-25$ & 19 & 315 & $30.8-76.3$ & 53.6 & 26.1 \\
\hline $\mathrm{n} / \mathrm{a}$ & $\mathrm{n} / \mathrm{a}$ & $\mathrm{n} / \mathrm{a}$ & $\mathrm{n} / \mathrm{a}$ & 350 & $58-66$ & 62.0 & $38.3-43.8$ \\
\hline $264-398$ & 331 & $13-21$ & 17 & 383 & $34.8-60.4$ & 47.6 & $26.3-27.8$ \\
\hline $452-472$ & 462 & 21 & 21 & 416 & 41.4 & 41.4 & $22.6-34.5$ \\
\hline 438 & 438 & $\mathrm{n} / \mathrm{a}$ & $\mathrm{n} / \mathrm{a}$ & 480 & $40-60$ & 50.0 & $\mathrm{n} / \mathrm{a}$ \\
\hline $\mathrm{n} / \mathrm{a}$ & $\mathrm{n} / \mathrm{a}$ & $\mathrm{n} / \mathrm{a}$ & $\mathrm{n} / \mathrm{a}$ & 337 & 56 & 56.0 & $\mathrm{n} / \mathrm{a}$ \\
\hline 357 & 357 & 16 & 16 & 150 & 50 & 50.0 & 30 \\
\hline
\end{tabular}


Ramphotyphlops marxi

Ramphotyphlops melanocephalus

Ramphotyphlops multilineatus

Ramphotyphlops olivaceus

Ramphotyphlops similis

Ramphotyphlops suluensis

Ramphotyphlops supranasalis

Ramphotyphlops willeyi

Sundatyphlops polygrammicus

Xerotyphlops etheridgei

Xerotyphlops socotranus

Xerotyphlops vermicularis

Xerotyphlops wilsoni

MADATYPHLOPINAE

Madatyphlops andasibensis

Madatyphlops arenarius

Madatyphlops boettgeri

Madatyphlops decorsei

Madatyphlops domerguei

Madatyphlops madagascariensis

Madatyphlops microcephalus

Madatyphlops mucronatus

Madatyphlops ocularis

Madatyphlops rajeryi

Madatyphlops reuteri

\section{TYPHLOPINAE}

Amerotyphlops amoipira

Amerotyphlops brongersmianus

Amerotyphlops costaricensis

Amerotyphlops lehneri

Amerotyphlops microstomus

Amerotyphlops minuisquamus

Amerotyphlops paucisquamus

Amerotyphlops reticulatus

Amerotyphlops stadelmani

Amerotyphlops tasymicris

Amerotyphlops tenuis

Amerotyphlops trinitatus

Amerotyphlops tycherus

Amerotyphlops yonenagae

Antillotyphlops annae

Antillotyphlops catapontus

Antillotyphlops dominicanus

Antillotyphlops geotomus

Antillotyphlops granti

Antillotyphlops guadeloupensis

Antillotyphlops hypomethes

Antillotyphlops monastus

Antillotyphlops monensis

Antillotyphlops naugus

Antillotyphlops platycephalus

Antillotyphlops richardi

Cubatyphlops anchaurus

Cubatyphlops anousius

Cubatyphlops arator

Cubatyphlops biminiensis

Cubatyphlops caymanensis

$\begin{array}{ccccc}525 & 525 & 36 & 36 & 180 \\ 524 & 524 & 20 & 20 & 183 \\ 513-586 & 550 & 20-22 & 21 & 427 \\ 441-544 & 493 & 24-31 & 27.5 & 425 \\ 234-235 & 235 & 9-12 & 10.5 & 235 \\ \mathrm{n} / \mathrm{a} & \mathrm{n} / \mathrm{a} & \mathrm{n} / \mathrm{a} & \mathrm{n} / \mathrm{a} & 340 \\ 340-346 & 343 & 16-18 & 17 & 301 \\ 369-375 & 372 & 11-15 & 13 & 195 \\ 496 & 496 & 16 & 16 & 395 \\ 424 & 424 & 10 & 10 & 220 \\ 435 & 435 & \mathrm{n} / \mathrm{a} & \mathrm{n} / \mathrm{a} & 255 \\ 206 & 206 & 16 & 16 & 350\end{array}$

$\begin{array}{lllll}n / a & n / a & n / a & n / a & 343\end{array}$

$\begin{array}{ccccc}373-381 & 377 & 8-11 & 9.5 & 340 \\ 375-465 & 420 & 7-11 & 9 & 220 \\ 349-436 & 393 & 7-12 & 9.5 & 226 \\ 421-600 & 511 & 9-13 & 11 & 600 \\ 252-262 & 257 & 6-7 & 6.5 & 176 \\ 580 & 580 & 15 & 15 & 410 \\ 310-365 & 338 & 8-11 & 9.5 & 235 \\ 488-577 & 533 & 13-18 & 15.5 & 418 \\ 523-579 & 551 & 16-18 & 17 & 404 \\ 412 & 412 & 12 & 12 & 272 \\ 345-359 & 352 & 8-9 & 8.5 & 222\end{array}$

$\begin{array}{cc}27.2-28.1 & 27.7 \\ 45-85 & 65.0 \\ 36-58 & 47.0 \\ 28-57 & 42.5 \\ 34-39 & 36.5 \\ 46 & 46.0 \\ 34-51 & 42.5 \\ 42-64 & 53.0 \\ 55-67 & 61.0 \\ 36.8 & 36.8 \\ 38-49 & 43.5\end{array}$

34.5-52.6 43.6

$50-111.1 \quad 80.6$

62.5-125 93.8

40-71.4 55.7

$43.5-55.6 \quad 49.6$

$46-51.3 \quad 48.7$

35.7-62.5 49.1

33.3-58.8 $\quad 46.1$

37-43.5 40.3

$36.7 \quad 36.7$

38-55.6 $\quad 46.8$

$\begin{array}{ccccccccc}212-242 & 227 & 7-12 & 9.5 & 208 & 22.8-27.7 & 25.3 & 36.5-70.7 & 53.6 \\ 204 & 204 & 12 & 12 & 325 & 24 & 24.0 & 30.6 & 30.6 \\ 390-413 & 402 & 7 & 7 & 369 & 36.4-44.4 & 40.4 & 57.8-78.1 & 68.0 \\ 289-337 & 313 & n / a & n / a & 185 & 31-58 & 44.5 & 71.9-125 & 98.5 \\ 487-556 & 522 & n / a & n / a & 366 & 50-77 & 63.5 & 73.5-270.3 & 171.9 \\ 219-253 & 236 & 6-11 & 8.5 & 361 & 20.4-33.7 & 27.1 & 31.3-52.6 & 42.0 \\ 170-190 & 180 & 8-11 & 9.5 & 158 & 16.4-21.8 & 19.1 & 26.6 & 26.6 \\ 223-301 & 262 & 7-15 & 11 & 522 & 22-30 & 26.0 & 23.3-62.5 & 42.9 \\ 341-369 & 355 & n / a & n / a & 310 & 37-70 & 53.5 & 43.9-100 & 72.0 \\ 429 & 429 & n / a & n / a & 181 & 48 & 48.0 & 70 & 70.0 \\ 347-441 & 394 & 7-11 & 9 & 326 & 34-74 & 54.0 & 42.2-91.7 & 67.0 \\ 388-389 & 389 & 10 & 10 & 240 & 50 & 50.0 & 76.9 & 76.9 \\ 395 & 395 & 8 & 8 & 371 & 40.8 & 40.8 & 66.2 & 66.2 \\ 259-269 & 264 & 9-10 & 9.5 & 101 & 25.3-27.4 & 26.4 & 48-50.5 & 49.3 \\ 400-405 & 403 & 14 & 14 & 110 & 57.9 & 57.9 & 61.1 & 61.1 \\ 376-409 & 393 & n / a & n / a & 265 & n / a & n / a & 37.9 & 37.9 \\ 434-499 & 467 & n / a & n / a & 360 & 40-50 & 45.0 & n / a & n / a \\ 329-367 & 348 & n / a & n / a & 213 & 43.6 & 43.6 & 39.2 & 39.2 \\ 370-386 & 378 & n / a & n / a & 154 & 54-70 & 62.0 & 59.2 & 59.2 \\ 393-430 & 412 & n / a & n / a & 162 & 36.8 & 36.8 & 46.3 & 46.3 \\ 363-407 & 385 & n / a & n / a & 270 & 41-56 & 48.5 & 35-47 & 41.0 \\ 351-394 & 373 & n / a & n / a & 258 & 39.1 & 39.1 & 30.7-43 & 36.9 \\ 299-345 & 322 & n / a & n / a & 203 & 40 & 40.0 & 60.7 & 60.7 \\ 345-390 & 368 & n / a & n / a & 243 & n / a & n / a & 30.4 & 30.4 \\ 350-365 & 358 & 11 & 11 & 302 & 45 & 45.0 & 39 & 39.0 \\ 300-369 & 335 & 12 & 12 & 258 & 50 & 50.0 & 35.7-39.7 & 37.7 \\ 514 & 514 & n / a & n / a & 240 & 63 & 63.0 & 48 & 48.0 \\ 465-513 & 489 & n / a & n / a & 197 & 45-55 & 50.0 & 55-56 & 55.5 \\ 578-579 & 579 & n / a & n / a & 460 & 51-55 & 53.0 & 46-48 & 47.0 \\ 454-537 & 496 & n / a & n / a & 380 & 39-58 & 48.5 & 42-76 & 59.0 \\ 351-408 & 380 & n / a & n / a & 260 & 36-45 & 40.5 & 29-35 & 32 \\ & & & & & & & & \end{array}$


Cubatyphlops contorhinus

Cubatyphlops epactius

Cubatyphlops golyathi

Cubatyphlops notorachius

Cubatyphlops paradoxus

Cubatyphlops perimychus

Cubatyphlops satelles

Typhlops agoralionis

Typhlops capitulatus

Typhlops eperopeus

Typhlops gonavensis

Typhlops hectus

Typhlops jamaicensis

Typhlops leptolepis

Typhlops lumbricalis

Typhlops oxyrhinus

Typhlops pachyrhinus

Typhlops proancylops

Typhlops pusillus

Typhlops rostellatus

Typhlops schwartzi

Typhlops silus

Typhlops sulcatus

Typhlops sylleptor

Typhlops syntherus

Typhlops tetrathyreus

Typhlops titanops

\begin{tabular}{ccccccccc}
502 & 502 & $\mathrm{n} / \mathrm{a}$ & $\mathrm{n} / \mathrm{a}$ & 316 & 63 & 63.0 & 53 & 53.0 \\
$473-505$ & 489 & $\mathrm{n} / \mathrm{a}$ & $\mathrm{n} / \mathrm{a}$ & 247 & $53-59$ & 56.0 & $40.3-47.5$ & 43.9 \\
629 & 629 & 22 & 22 & 371 & 58 & 58.0 & 33.7 & 33.7 \\
$475-529$ & 502 & $\mathrm{n} / \mathrm{a}$ & $\mathrm{n} / \mathrm{a}$ & 301 & $45-57$ & 51.0 & $48-75$ & 61.5 \\
$455-472$ & 464 & $\mathrm{n} / \mathrm{a}$ & $\mathrm{n} / \mathrm{a}$ & 245 & $\mathrm{n} / \mathrm{a}$ & $\mathrm{n} / \mathrm{a}$ & $47-49$ & 48.0 \\
$453-496$ & 475 & $\mathrm{n} / \mathrm{a}$ & $\mathrm{n} / \mathrm{a}$ & 280 & $41-59$ & 50.0 & $46-85$ & 65.5 \\
$514-527$ & 521 & $\mathrm{n} / \mathrm{a}$ & $\mathrm{n} / \mathrm{a}$ & 350 & $62-75$ & 68.5 & $55-61$ & 58.0 \\
$291-310$ & 301 & $\mathrm{n} / \mathrm{a}$ & $\mathrm{n} / \mathrm{a}$ & 193 & $31-39$ & 35.0 & $27-31$ & 29.0 \\
$358-457$ & 408 & $\mathrm{n} / \mathrm{a}$ & $\mathrm{n} / \mathrm{a}$ & 267 & $46-57$ & 51.5 & $29-46$ & 37.5 \\
$305-329$ & 317 & $\mathrm{n} / \mathrm{a}$ & $\mathrm{n} / \mathrm{a}$ & 281 & $29-39$ & 34.0 & $27-31$ & 29.0 \\
$399-455$ & 427 & $\mathrm{n} / \mathrm{a}$ & $\mathrm{n} / \mathrm{a}$ & 220 & $46-57$ & 51.5 & $38-88$ & 63.0 \\
$284-328$ & 306 & $\mathrm{n} / \mathrm{a}$ & $\mathrm{n} / \mathrm{a}$ & 230 & $31-46$ & 38.5 & $20-44$ & 32.0 \\
$373-436$ & 405 & $\mathrm{n} / \mathrm{a}$ & $\mathrm{n} / \mathrm{a}$ & 445 & $27-44$ & 35.5 & $\mathrm{n} / \mathrm{a}$ & $\mathrm{n} / \mathrm{a}$ \\
$250-308$ & 279 & $11-15$ & 13 & 211 & $29.6-43.7$ & 36.7 & $18-37$ & 27.5 \\
$256-271$ & 264 & $12-13$ & 12.5 & 166 & $28-32.6$ & 30.3 & $30.8-48$ & 39.4 \\
$265-297$ & 281 & $10-16$ & 13 & 257 & $26.8-46.6$ & 36.7 & $19.8-37.5$ & 28.7 \\
$243-257$ & 250 & $11-14$ & 12.5 & 219 & $28.4-33$ & 30.7 & $30.1-31.3$ & 30.7 \\
$283-312$ & 298 & $\mathrm{n} / \mathrm{a}$ & $\mathrm{n} / \mathrm{a}$ & 243 & $31-46$ & 38.5 & $20-31$ & 25.5 \\
$245-332$ & 289 & $15-19$ & 17 & 226 & $27-37$ & 32.0 & $23-39$ & 31.0 \\
$314-358$ & 336 & 13 & 13 & 222 & $32-52$ & 42.0 & $33-66.7$ & 49.9 \\
$237-282$ & 260 & $\mathrm{n} / \mathrm{a}$ & $\mathrm{n} / \mathrm{a}$ & 326 & $23-38$ & 30.5 & $22-41$ & 31.5 \\
$254-261$ & 258 & $\mathrm{n} / \mathrm{a}$ & $\mathrm{n} / \mathrm{a}$ & 126 & $30-34$ & 32.0 & $56.7-63$ & 59.9 \\
$371-447$ & 409 & $\mathrm{n} / \mathrm{a}$ & $\mathrm{n} / \mathrm{a}$ & 319 & $37-44$ & 40.5 & $36-60$ & 48.0 \\
$305-324$ & 315 & $\mathrm{n} / \mathrm{a}$ & $\mathrm{n} / \mathrm{a}$ & 214 & $33-34$ & 33.5 & $27-43$ & 35.0 \\
$299-353$ & 326 & $8-14$ & 11 & 209 & $25-36$ & 30.5 & $27-51$ & 39.0 \\
$246-294$ & 270 & $\mathrm{n} / \mathrm{a}$ & $\mathrm{n} / \mathrm{a}$ & 273 & $25-38$ & 31.5 & $23-47$ & 35.0 \\
$231-264$ & 248 & $\mathrm{n} / \mathrm{a}$ & $\mathrm{n} / \mathrm{a}$ & 216 & $25-30$ & 27.5 & $21-34$ & 27.5 \\
\hline & & & & & & & &
\end{tabular}

\section{AFROTYPHLOPINAE}

Afrotyphlops angolensis

Afrotyphlops anomalus

Afrotyphlops bibronii

Afrotyphlops blanfordii

Afrotyphlops brevis

Afrotyphlops calabresii

Afrotyphlops comorensis

Afrotyphlops congestus

Afrotyphlops cuneirostris

Afrotyphlops decorosus

Afrotyphlops elegans

Afrotyphlops fornasinii

Afrotyphlops gierrai

Afrotyphlops jubanus

Afrotyphlops kaimosae

Afrotyphlops liberiensis

Afrotyphlops lineolatus

Afrotyphlops mucruso

Afrotyphlops nanus

Afrotyphlops nigrocandidus

Afrotyphlops obtusus

Afrotyphlops platyrhynchus

Afrotyphlops punctatus

Afrotyphlops rondoensis

Afrotyphlops schlegelii

Afrotyphlops schmidti

\section{7}

gy,dk-gy

bn,ol-bn

yl-bn,or-bn,dk-bn,bk

$$
\text { ol-gy }
$$

gn-bn,gy-bn,gd-bn

$$
\text { rd-bn }
$$

dk-bn,rd-bn

bn-bk,yl

rd-bn

oc-yl

or-yl

dk-gy,bk

pl-bn,bl-gy

bn, dk-bn

bk,dk-bn

$\mathrm{n} / \mathrm{a}$

yl,bk

bk

pl-yl,cr-yl

bk,bl-bk,wt,yl

bn,dr-bn,bk

rd-yl

bn,pu,gy,yl,dk-bn

bl-gy,bk

bk

dk-bn,bk
8

\begin{tabular}{|c|c|c|}
\hline yl & 1 & $\mathrm{rt}$ \\
\hline yl & 1 & $\mathrm{n} / \mathrm{a}$ \\
\hline oc-yl,bn,dk-bn,bk & 0,1 & pl-dl \\
\hline pl & 1 & $\mathrm{vl}$ \\
\hline$y l, p l$ & 1 & dl(juv) \\
\hline wt & 1 & $8-12 d k-d l$ \\
\hline $\mathrm{n} / \mathrm{a}$ & $\mathrm{n} / \mathrm{a}$ & $\mathrm{n} / \mathrm{a}$ \\
\hline yl & 1 & bk-bl \\
\hline wt & 1 & $8 \mathrm{dk}-\mathrm{dl}$ \\
\hline oc-yl & 1 & $8-10 \mathrm{dk}-\mathrm{dl}$ \\
\hline or-yl & 1 & bk-dl \\
\hline dk-gy,bk & 1 & yl-bl (ven) \\
\hline yl-cr & 1 & $\mathrm{n} / \mathrm{a}$ \\
\hline pl & 1 & $\mathrm{n} / \mathrm{a}$ \\
\hline $\mathrm{pl}$ & 1 & $\mathrm{n} / \mathrm{a}$ \\
\hline $\mathrm{n} / \mathrm{a}$ & $\mathrm{n} / \mathrm{a}$ & $\mathrm{n} / \mathrm{a}$ \\
\hline yl,pl & 1 & bn-dl,bl(ven \\
\hline wt & 1 & dl,bl \\
\hline pl-yl,cr-yl & 1 & $d k-d l$ \\
\hline wt,yl & 1 & bk-bl \\
\hline yl,pl-bn,wt & 1 & wt-dl \\
\hline pl-rd-yl & 1 & $\mathrm{n} / \mathrm{a}$ \\
\hline dk-bn,gy,pl,yl & 1 & yl-bk-bl-sp \\
\hline wt,yl & 1 & $\mathrm{dl}$ \\
\hline pl & $1(0)$ & bl,dk-vl \\
\hline $\mathrm{pl}$ & 1 & $\mathrm{dl}, \mathrm{sp}$ \\
\hline
\end{tabular}

20 
Afrotyphlops steinhausi

Afrotyphlops tanganicanus

Afrotyphlops usambaricus

Letheobia acutirostrata

Letheobia angeli

Letheobia caeca

Letheobia coecata

Letheobia crossii

Letheobia debilis

Letheobia episcopa

Letheobia erythraea

Letheobia feae

Letheobia gracilis

Letheobia graueri

Letheobia kibarae

Letheobia largeni

Letheobia leucosticta

Letheobia lumbriciformis

Letheobia manni

Letheobia newtoni

Letheobia pallida

Letheobia pauwelsi

Letheobia pembana

Letheobia praeocularis

Letheobia rufescens

Letheobia simonii

Letheobia somalica

Letheobia stejnegeri

Letheobia sudanensis

Letheobia swahilica

Letheobia toritensis

Letheobia uluguruensis

Letheobia wittei

Letheobia zenkeri

Rhinotyphlops ataeniatus

Rhinotyphlops boylei

Rhinotyphlops lalandei

Rhinotyphlops leucocephalus

Rhinotyphlops schinzi

Rhinotyphlops scortecci

Rhinotyphlops unitaeniatus

\section{ASIATYPHLOPINAE}

Acutotyphlops banaorum Acutotyphlops infralabialis Acutotyphlops kunuaensis Acutotyphlops solomonis Acutotyphlops subocularis

Anilios affinis

Anilios ammodytes

Anilios aspinus

Anilios australis

Anilios batillus

Anilios bicolor

Anilios bituberculatus

Anilios broomi

Anilios centralis

Anilios chamodracaena dk-bn

yl,bn

yl

$\mathrm{n} / \mathrm{a}$

$\mathrm{n} / \mathrm{a}$

pl-bn,pl

bn

pl

pk-tn

yl-cr

yl-wt

$\mathrm{pl}$

pl

$\mathrm{pl}$

pk

wt,cr-wt

$\mathrm{dk}-\mathrm{bn}$

pl

gy

yl-wt

pl

bg

pl

bn-gy

pl-rd,pl

yl-pl,pl

ol,pl

yl-wt

pl-yl-bn,pl

pl

$\mathrm{pl}$

pl

pl

$\mathrm{p}$

bk,yl-bn,gn-bn

ol-bn

oc-yl,pl-bn,bn,gy,gy-bn

gy

$y \mathrm{l}, \mathrm{rd}, \mathrm{pk}, \mathrm{bk}$

dk-bn,bk

bk,dk-bn

gd-or,pl-or,or-bn,bn

bn, dk

$\mathrm{bn}, \mathrm{dk}$

bn, dk

bn, dk

ol-bn,dk-bn

pl-gy-bn,pk-pu

pl-pk-tn

pl,pl-yl,rd-bn,gy-bn or-bn

bn,pu-bn,dk-bn,gy-bn

bn-um,dk-pu-bn

pl,pl-bn

pu-bn

yl-cr pl-yl,pl

yl-bn

yl

$\mathrm{n} / \mathrm{a}$

$\mathrm{n} / \mathrm{a}$

pl-bn,pl

pl-bn

pl

pk-tn

yl-cr

yl-wt

pl

pl

$\mathrm{pl}$

pl-pk

cr-wt

wt

pl

yl-gy

yl-wt

pl

$\mathrm{cr}, \mathrm{pl}$

pl

pl-bn-gy

pl-rd,pl

yl-pl,pl

pl-ol,pl

yl-wt

pl-yl-bn,pl

pl

pl

pl

pl

$\mathrm{pl}$

bk,yl-bn,gn-bn

yl

yl,pl,wt

wt

pl

dk-bn,bk

bn-yl

gd,bn-or

yl

pl-yl

gd

yl

pl-yl,pl-yl-cr,pk-wt

pl-gy-bn,pk-pu

pl-pk-tn

cr,wt

pl-or-bn

gn-wt,yl-wt,cr,wt

wt

wt

pl

yl-cr,wt yl-br

$\mathrm{n} / \mathrm{a}$

bk-bl

$\mathrm{n} / \mathrm{a}$

$\mathrm{n} / \mathrm{a}$

$\mathrm{n} / \mathrm{a}$

$\mathrm{n} / \mathrm{a}$

$\mathrm{n} / \mathrm{a}$

$\mathrm{n} / \mathrm{a}$

$\mathrm{n} / \mathrm{a}$

$\mathrm{n} / \mathrm{a}$

$n / a$

$\mathrm{n} / \mathrm{a}$

$\mathrm{n} / \mathrm{a}$

$\mathrm{n} / \mathrm{a}$

$\mathrm{n} / \mathrm{a}$

wt-sp

$\mathrm{n} / \mathrm{a}$

bk-sp(ven)

$\mathrm{n} / \mathrm{a}$

$\mathrm{n} / \mathrm{a}$

$\mathrm{n} / \mathrm{a}$

$\mathrm{n} / \mathrm{a}$

$\mathrm{n} / \mathrm{a}$

$\mathrm{n} / \mathrm{a}$

dl

$\mathrm{n} / \mathrm{a}$

$n / a$

$\mathrm{n} / \mathrm{a}$

$\mathrm{n} / \mathrm{a}$

$\mathrm{n} / \mathrm{a}$

$\mathrm{n} / \mathrm{a}$

$\mathrm{n} / \mathrm{a}$

$\mathrm{n} / \mathrm{a}$

or-yl-dl(head)

$\mathrm{n} / \mathrm{a}$

$\mathrm{n} / \mathrm{a}$

bk-dl

dk-sp-br

$y l-d l$

$y l-d l$

bk-sp-br

dk-dl

yl-nk

$\mathrm{n} / \mathrm{a}$

$\mathrm{n} / \mathrm{a}$

$\mathrm{n} / \mathrm{a}$

$\mathrm{n} / \mathrm{a}$

$n / a$

$\mathrm{n} / \mathrm{a}$

dl

$\mathrm{n} / \mathrm{a}$

$\mathrm{n} / \mathrm{a}$

11 bn-dl

$\mathrm{n} / \mathrm{a}$

$\mathrm{n} / \mathrm{a}$ 
Anilios diversus

Anilios endoterus

Anilios erycinus

Anilios ganei

Anilios grypus

Anilios guentheri

Anilios hamatus

Anilios howi

Anilios kimberleyensis

Anilios leptosomus

Anilios leucoproctus

Anilios ligatus

Anilios longissimus

Anilios margaretae

Anilios micrommus

Anilios minimus

Anilios nema

Anilios nigrescens

Anilios nigroterminatus

Anilios pilbarensis

Anilios pinguis

Anilios proximus

Anilios robertsi

Anilios silvia

Anilios splendidus

Anilios torresianus

Anilios tovelli

Anilios troglodytes

Anilios unguirostris

Anilios waitii

Anilios wiedii

Anilios yampiensis

Anilios yirrikalae

Asiatyphlops bothriorhynchus

Asiatyphlops diardii

Asiatyphlops fuscus

Asiatyphlops giadinhensis

Asiatyphlops klemmeri

Asiatyphlops koshunensis

Asiatyphlops leucomelas

Asiatyphlops muelleri

Asiatyphlops oatesii

Asiatyphlops roxaneae

Asiatyphlops siamensis

Asiatyphlops tenuicollis

Asiatyphlops trangensis

Cyclotyphlops deharvengi

Grypotyphlops acutus

Indotyphlops ahsanai

Indotyphlops albiceps

Indotyphlops braminus

Indotyphlops exiguus

Indotyphlops filiformis

Indotyphlops fletcheri

Indotyphlops hypsobothrius

Indotyphlops jerdoni

Indotyphlops khoratensis bn,bk-bn,pu-bn

bn,dk-pu-bn,rd-bn

bn-gy,bn

gy-bn

bn,pk-bn,bn-cr

rd-bn,pu-bn

bn-bk,pu-bn

dk-bn

$\mathrm{dk}$

pl-gy-bn,pu-bn

bn, dk-bn,dk-tn

bn,dk-bn,dk-pu-bn

$$
\text { pl }
$$

pk,pu-gy

$\mathrm{n} / \mathrm{a}$

yl-bn

pl-yl-bn,gy-bn

bk,pu-pk-bn

pl-bn

pl-pu-bn

bn-yl,dk-bn

bn-ol,gy-bn

dk-pu-bn

pu-bk,cm

gy

dk-bn,ol

bn

bn

ol-bn,dk-ol-bn

pl-bn,dk-pu-bn

rd-bn,bk-bn

bn

bn,yl

bn-ol,bn

ol-bn,bn,bk

dk-bn,bn

gd-bn

bn

n/a

bk-bn,bk

bk

yl,bn

gd-bn

gy-ol

ol-bn

bl

$d k-b n$

or-gy,bn,gd-bn

dk-bn

bn, pl-bn

dk-gy,bn,bk

pl-yl

dk-rd-bn

dk-pu-bn

pl-bn

pl-bn,bn,bk

gy

wt,pl-pu-bn
wt,cr
yl
pl
gy-wt

pl-rd-bn,pl-pu-bn

wt,cr

bn

$\mathrm{pl}$

gy-wt,pl-pu-bn

dk-bn

yl-wt,wt

pl

pl-gy

$\mathrm{n} / \mathrm{a}$

yl-bn

pl-yl-bn,pl-gy-bn

$\mathrm{cr}, \mathrm{pk}$

pl-bn,yl

$\mathrm{cm}$

pl-bn-yl,wt

yl

$\mathrm{cr}$

cr,cr-yl

wt

wt,yl

wt

pl

yl,wt

$y l, w t$

yl,cr-wt

pl-bn

$w t, y l$

bn-ol,pl-bn

bn,yl,pl-bn

dk-bn

yl

pl-yl-bn

n/a

wt

cr-wt

yl-bn

yl-tn

yl

yl

cr-wt

bn

gy-wt,yl,pl-bn

$\mathrm{n} / \mathrm{a}$

cr-wt

pl-gy,pl-bn

wt

rd-bn

pu-bn

wt

pl-bn

gy $\mathrm{n} / \mathrm{a}$

$\mathrm{n} / \mathrm{a}$

dk-dl

$\mathrm{n} / \mathrm{a}$

$\mathrm{n} / \mathrm{a}$

$n / a$

$\mathrm{n} / \mathrm{a}$

$n / a$

$n / a$

$\mathrm{n} / \mathrm{a}$

$\mathrm{n} / \mathrm{a}$

$\mathrm{n} / \mathrm{a}$

$n / a$

$n / a$

$\mathrm{n} / \mathrm{a}$

$\mathrm{n} / \mathrm{a}$

$\mathrm{n} / \mathrm{a}$

$\mathrm{n} / \mathrm{a}$

$\mathrm{n} / \mathrm{a}$

$\mathrm{n} / \mathrm{a}$

$\mathrm{n} / \mathrm{a}$

n/a

$\mathrm{n} / \mathrm{a}$

$11 \mathrm{dk}-\mathrm{dl}$

$\mathrm{n} / \mathrm{a}$

$\mathrm{n} / \mathrm{a}$

dl

n/a

$\mathrm{n} / \mathrm{a}$

$\mathrm{n} / \mathrm{a}$

$\mathrm{n} / \mathrm{a}$

n/a

n/a

n/a

$\mathrm{n} / \mathrm{a}$

$\mathrm{n} / \mathrm{a}$

n/a

vl

$\mathrm{n} / \mathrm{a}$

$\mathrm{n} / \mathrm{a}$

$n / a$

dk-dl-sp

n/a

n/a

$\mathrm{n} / \mathrm{a}$

$\mathrm{n} / \mathrm{a}$

$\mathrm{n} / \mathrm{a}$

dl

n/a

$\mathrm{n} / \mathrm{a}$

$\mathrm{n} / \mathrm{a}$

n/a

n/a

n/a

$\mathrm{n} / \mathrm{a}$

n/a

n/a 


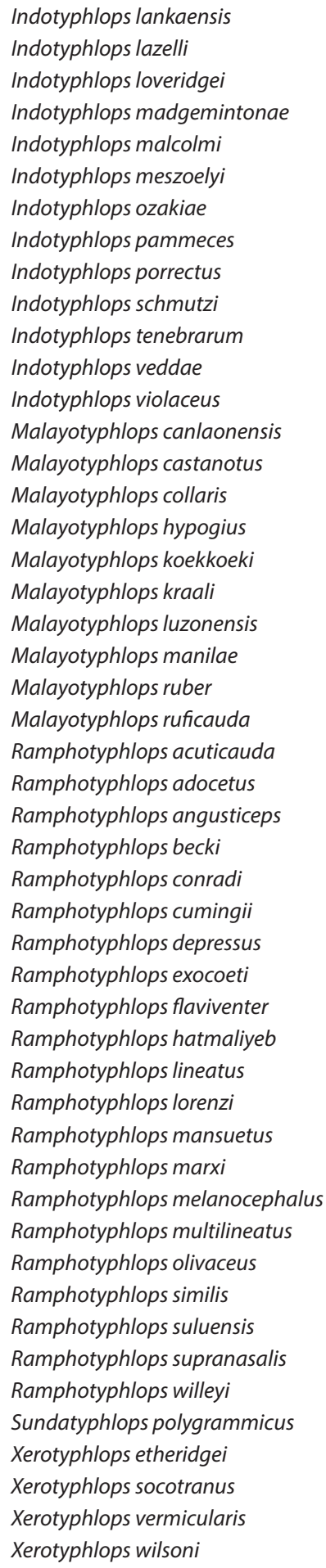

pl-bn

pl-bn

pl-bn

dk-bn,yl-bn

bn

bn

$\mathrm{n} / \mathrm{a}$

$\mathrm{n} / \mathrm{a}$

bn, bk

pk-bn

bn

pu-gy

pu

gn-bk

dk-bn

pl-bn

pl-gy-bn

gy-bn

bk,bk-bn

rd-ol-bn

rd-bn

rd-bn

bk,rd-bn

bn

dk-bn

bn

rd-bn,dk-pu-bn

pl-bn

pl-ol,bk-gy,ol-bn

bn

pl-bn

dk-bn,bk,dk-pu

dk-bn

yl,pl-bn,bk

pl-gy-gn

bn

pl-bn

bk-bn

bn-gy,gy,bn

dk-ol,pl-ol-bn

bn, dk-bn

dk-gy

dk-bn,dk-pu,bk

ol-bn,bn

dk-bn,ol,ol-gy,pl-yl

pl-bn

wt

bn,yl-bn,pk-bn,pu-bn bn

bn

pk-wt,pl

pl

dk-gy

pl-br

bk-bn,bn

bk pl-bn

wt

pl-bn

pl-bn,yl

$\mathrm{pl}$

pl-bn

$\mathrm{n} / \mathrm{a}$

n/a

pl-bn

pk-bn

pk-bn

pu-gy

$\mathrm{pu}$

pk-yl

cr,pl

pl

pl-yl

pl-gy-bn

yl

yl-bn

yl-wt

rd-bn

$r d, y l$

yl

pl

yl

pl-bn

pl-bn

$\mathrm{pl}, \mathrm{yl}$

$y l, g d$

pl-bn

yl

pl

yl-wt

pl-gy-gn

bn

yl-cr

$\mathrm{pl}$

br-gy,gy,bn

yl,pl-ol-bn

yl

pl-gy

yl

yl,pl-bn

wt,wt-pk,pl-yl

pl-yl-wt,bg

wt

bn,yl-bn,pk-bn,pu-bn

bn

bn
pk-wt,pl
pl,bg
gy-wt
pl-bn
bk-bn
bk

$n / a$

$\mathrm{n} / \mathrm{a}$

n/a

n/a

dk-nk

$n / a$

$\mathrm{n} / \mathrm{a}$

n/a

$n / a$

n/a

$\mathrm{n} / \mathrm{a}$

$\mathrm{n} / \mathrm{a}$

$\mathrm{n} / \mathrm{a}$

n/a

n/a

pl-nk

dk-dl

n/a

yl-sp(ven)

$\mathrm{n} / \mathrm{a}$

n/a

n/a

$\mathrm{n} / \mathrm{a}$

n/a

n/a

$\mathrm{n} / \mathrm{a}$

$\mathrm{n} / \mathrm{a}$

$n / a$

n/a

$\mathrm{n} / \mathrm{a}$

dl

n/a

$\mathrm{n} / \mathrm{a}$

yl-bn-dl

$\mathrm{n} / \mathrm{a}$

$\mathrm{n} / \mathrm{a}$

n/a

n/a

yl-bn-dl

dl

$\mathrm{n} / \mathrm{a}$

rt

$\mathrm{n} / \mathrm{a}$

$\mathrm{n} / \mathrm{a}$

$\mathrm{dl}, \mathrm{vl}, \mathrm{un}$

$\mathrm{n} / \mathrm{a}$

pl-bn-dl

$\mathrm{n} / \mathrm{a}$

$\mathrm{n} / \mathrm{a}$

yl-nk,yl-sp(ven)

$\mathrm{n} / \mathrm{a}$

$\mathrm{n} / \mathrm{a}$

$\mathrm{n} / \mathrm{a}$

$\mathrm{n} / \mathrm{a}$

pl-dl

yl-vl 
Madatyphlops mucronatus

Madatyphlops ocularis

Madatyphlops rajeryi

Madatyphlops reuteri

TYPHLOPINAE

Amerotyphlops amoipira

Amerotyphlops brongersmianus

Amerotyphlops costaricensis

Amerotyphlops lehneri

Amerotyphlops microstomus

Amerotyphlops minuisquamus

Amerotyphlops paucisquamus

Amerotyphlops reticulatus

Amerotyphlops stadelmani

Amerotyphlops tasymicris

Amerotyphlops tenuis

Amerotyphlops trinitatus

Amerotyphlops tycherus

Amerotyphlops yonenagae

Antillotyphlops annae

Antillotyphlops catapontus

Antillotyphlops dominicanus

Antillotyphlops geotomus

Antillotyphlops granti

Antillotyphlops guadeloupensis

Antillotyphlops hypomethes

Antillotyphlops monastus

Antillotyphlops monensis

Antillotyphlops naugus

Antillotyphlops platycephalus

Antillotyphlops richardi

Cubatyphlops anchaurus

Cubatyphlops anousius

Cubatyphlops arator

Cubatyphlops biminiensis

Cubatyphlops caymanensis

Cubatyphlops contorhinus

Cubatyphlops epactius

Cubatyphlops golyathi

Cubatyphlops notorachius

Cubatyphlops paradoxus

Cubatyphlops perimychus

Cubatyphlops satelles

Typhlops agoralionis

Typhlops capitulatus

Typhlops eperopeus

Typhlops gonavensis

Typhlops hectus

Typhlops jamaicensis

Typhlops leptolepis

Typhlops lumbricalis

Typhlops oxyrhinus

Typhlops pachyrhinus

Typhlops proancylops

Typhlops pusillus

Typhlops rostellatus

Typhlops schwartzi

$$
\begin{aligned}
& \text { gy-bn,ol,bn } \\
& \text { pl-bn-gy,bn } \\
& \text { yl,gb } \\
& \text { gy,gy-bl,bn }
\end{aligned}
$$

cr-bn

pl-bn

bn

pl-bn,yl

pl,pk-wt,yl-ol

bn,bk-bn,yl-bn

$$
\mathrm{n} / \mathrm{a}
$$

bn, dk-bn,bk

pl-yl

bn

bn,pl-bn,yl

$$
\text { yl }
$$

bn-gy

$\mathrm{cr}$

pk-gy-bg

gy-bn

bk-bn

gy-bn

yl,pl-bn

pl-bn

pl-gy-bn

gy-bn

bn,pl-bn

dk-bn

rd-bn,dk-bn

pl-bn

pl

pl

pl-bn

bn,pl-bn

bn

bn

pl-gy-bn,pl-rd,gy-bn

dk-bn

pl-bn

dk-bn

bn,dk-bn

pl-bn

pl-bn

bn,pl-bn,dk-rd-bn

bn,pl-tn,dk-bn

dk-pu-bn,dk-rd-bn

pl-gy,bn,pl-bn,tn

bn,dk-bn,pl-bn,gy-bn

dk-um,dk-bn

dk-bn

pl-bn

dk-bn

$\mathrm{n} / \mathrm{a}$

bn,pl-bn,dk-bn

dk-bn

dk-bn

$\begin{array}{ccc}\text { gy-bn,ol } & 1 & \text { n/a } \\ \text { pl-bn-gy } & 1 & n / a \\ \text { yl,bg } & 1 & n / a \\ \text { pl-gy,pl-gy-bl } & 1 & n / a\end{array}$

$\begin{array}{cll}\text { cr-bn } & 0 & \text { rt } \\ \text { pl-tn } & 1 & \text { rt } \\ \text { pl } & 1 & \text { bk-sp,tr } \\ \text { gy,yl } & 1 & 8-10 \text { dk-dl } \\ \text { pl,pk,wt } & 0 & \mathrm{n} / \mathrm{a} \\ \text { pl } & 1 & \mathrm{dl}, \mathrm{sp} \\ \mathrm{pl} & 1 & \mathrm{n} / \mathrm{a}\end{array}$

pl-yl,yl,wt,pl

pl-yl

pl

wt,yl

yl

pl-yl-gy,wt,p

$\mathrm{cr}$

$\mathrm{cr}$

pl-pk,pl

bk-bn

wt

yl-wt,pl

wt

pl

pl

wt

gy

wt,bg

wt,yl-wt

pl

pl

pl

pl,yl-wt

pl,yl-wt

pl

pl

pl-bn

pl

pl

pl

pl

pl-bn

pl-bn,dk-rd-bn

pl-tn,pl-bn

pu-bn,rd-bn

pl,pl-tn,pl-gy

pl

dk-um,dk-bn

It-bn,cr

pl-bn

pl-bn,cr

$\mathrm{n} / \mathrm{a}$

cr,gy-wt,pl

dk-bn

pl wt-tr

$\mathrm{n} / \mathrm{a}$

dk-dl

sp

11 dk-bn-dl

$\mathrm{n} / \mathrm{a}$

dk-bn-dl,bn-bl

$\mathrm{n} / \mathrm{a}$

pl-tr

$\mathrm{n} / \mathrm{a}$

$\mathrm{n} / \mathrm{a}$

$\mathrm{n} / \mathrm{a}$

$\mathrm{n} / \mathrm{a}$

$\mathrm{n} / \mathrm{a}$

$\mathrm{n} / \mathrm{a}$

dl

dl

$\mathrm{n} / \mathrm{a}$

$\mathrm{n} / \mathrm{a}$

$\mathrm{n} / \mathrm{a}$

$\mathrm{n} / \mathrm{a}$

$\mathrm{n} / \mathrm{a}$

$\mathrm{n} / \mathrm{a}$

$\mathrm{n} / \mathrm{a}$

$\mathrm{n} / \mathrm{a}$

$\mathrm{n} / \mathrm{a}$

$\mathrm{n} / \mathrm{a}$

$\mathrm{n} / \mathrm{a}$

$\mathrm{n} / \mathrm{a}$

$\mathrm{n} / \mathrm{a}$

$\mathrm{n} / \mathrm{a}$

$\mathrm{n} / \mathrm{a}$

$\mathrm{n} / \mathrm{a}$

$\mathrm{n} / \mathrm{a}$

$\mathrm{n} / \mathrm{a}$

dk-nk

rt

$\mathrm{n} / \mathrm{a}$

dk-nk

$\mathrm{n} / \mathrm{a}$

$\mathrm{n} / \mathrm{a}$

$\mathrm{n} / \mathrm{a}$

$\mathrm{n} / \mathrm{a}$

bk-rt

$\mathrm{n} / \mathrm{a}$ 
caribbean herpetology

article

Typhlops silus

Typhlops sulcatus

Typhlops sylleptor

Typhlops syntherus

Typhlops tetrathyreus

Typhlops titanops pl-cr-bn,bn-gy

bn,pl-bn,dk-bn

bn

bn-tn,bn,pl-bn,pl-tn

tn, bn

gy-bn,tn pl-cr-bn,pl-bn-gy

pl,pl-bn

pl-bn,pl

wt

pl

pl n/a

dk-dl

dk-nk

pl-bl,dl

n/a

$\mathrm{n} / \mathrm{a}$

Table 2. A summary of morphological variation in the subfamilies and genera of typhlopid snakes. See Table 1 legend for abbreviations.

\begin{tabular}{|c|c|c|c|c|c|c|c|c|c|c|c|}
\hline Taxon & Species & 1 & 2 & 3 & 4 & 5 & 6 & 7 & $8 a$ & $8 \mathrm{~b}$ & 9 \\
\hline Afrotyphlopinae & 67 & $d, i$ & $b, r(a)$ & nc & a & $c, i$ & $1,2(r, s 1)$ & $a, p$ & $2-6(7)$ & 3.68 & $2-3(1-2,1-3,2,3,2-4,0)$ \\
\hline Afrotyphlops & 29 & $d$ & $r(b, a)$ & nc & a & $\mathrm{i}(\mathrm{c})$ & $1(r, 2)$ & $a(p)$ & $2-4(5-7)$ & 3.70 & $2-3(1-2,1-3,2,0)$ \\
\hline Letheobia & 31 & $\mathrm{i}$ & $b, r$ & $\mathrm{nc}$ & a & $c, i$ & $2(r, 1, s 1, p)$ & $\mathrm{p}(\mathrm{a})$ & $3-4(2,5-7)$ & 3.55 & $2-3(2,3,2-4,0)$ \\
\hline Rhinotyphlops & 7 & $d$ & $b(r)$ & nc & a & $c, i$ & $2(1)$ & $a(p)$ & $4-6(2-3)$ & 4.21 & $2-3(2,2-4)$ \\
\hline Asiatyphlopinae & 121 & $d(i)$ & $r(b, a)$ & $\mathrm{nc}(\mathrm{c})$ & $a(p)$ & $c, i$ & $2(1, s 1, s 2, P)$ & $a, p$ & $1-5$ & 2.19 & $2-3(3,0)$ \\
\hline Acutotyphlops & 5 & d & $a, r$ & $\mathrm{nc}$ & $\mathrm{p}$ & $\mathrm{i}$ & 2(1) & $\mathrm{p}(\mathrm{a})$ & $3-5$ & 4.10 & $2-3,3,0$ \\
\hline Anilios & 43 & $d$ & $b, r(a)$ & nc & a & $c, i$ & $2(1, s 1, s 2, P)$ & $\mathrm{a}$ & $2(1,3-4)$ & 2.21 & $2-3$ \\
\hline Asiatyphlops & 13 & $d(i)$ & $r$ & nc & a & $\mathrm{i}(\mathrm{c})$ & 2 & a & $2-3(4-5)$ & 2.69 & $2-3(3)$ \\
\hline Cyclotyphlops & 1 & $d$ & $r$ & c & a & c & s1 & $\mathrm{p}$ & 1 & 1.00 & 0 \\
\hline Grypotyphlops & 1 & $d, i$ & b & $\mathrm{nc}$ & a & $c, i$ & 2 & $\mathrm{p}$ & $4(3,5)$ & 4.00 & $2-3$ \\
\hline Indotyphlops & 22 & $d(i)$ & $r$ & $\mathrm{nc}$ & a & $c, i$ & $2(1, p)$ & a & $1(2)$ & 1.03 & $2-3,3$ \\
\hline Malayotyphlops & 10 & $d(i)$ & $r$ & $\mathrm{nc}$ & a & $\mathrm{i}(\mathrm{c})$ & $2(1)$ & $a(p)$ & $2-4$ & 2.75 & $3,2-3(2)$ \\
\hline Ramphotyphlops & 21 & $d(i)$ & $b, r$ & $\mathrm{nc}$ & a & $c, i$ & $2(1,51)$ & $a(p)$ & $2-3(1)$ & 2.15 & $2-3(3)$ \\
\hline Sundatyphlops & 1 & $d$ & $r$ & $\mathrm{nc}$ & a & $c, i$ & $1, s 1,2$ & $\mathrm{n} / \mathrm{a}$ & $\mathrm{n} / \mathrm{a}$ & $\mathrm{n} / \mathrm{a}$ & $2-3$ \\
\hline Xerotyphlops & 4 & $d$ & $r$ & $\mathrm{nc}$ & a & $\mathrm{i}$ & 2 & $a(p)$ & 2 & 2.00 & $2-3$ \\
\hline Madatyphlopinae & 11 & $d$ & r & $\mathrm{nc}$ & a & $\mathrm{i}(\mathrm{c})$ & 2 & a & $2-3(1,4)$ & 2.27 & $2-3$ \\
\hline Madatyphlops & 11 & $d$ & r & $\mathrm{nc}$ & a & $\mathrm{i}(\mathrm{c})$ & 2 & a & $2-3(1,4)$ & 2.27 & $2-3$ \\
\hline Typhlopinae & 58 & $d(i)$ & $r(a)$ & $\mathrm{nc}$ & a & $c(i)$ & $2(1,51)$ & $a(p)$ & $1-4$ & 1.66 & $2-3,3(0,4 ?)$ \\
\hline Amerotyphlops & 14 & $d(i)$ & $r$ & $\mathrm{nc}$ & a & $c, i$ & $2(1,51)$ & $a(p)$ & $1-2(3,4)$ & 1.69 & $2-3$ \\
\hline Antillotyphlops & 12 & $d$ & $r(a)$ & $\mathrm{nc}$ & a & c & 2 & a & $2(1)$ & 2.00 & $3(4 ?)$ \\
\hline Cubatyphlops & 12 & $d$ & $r$ & $\mathrm{nc}$ & a & c & 2 & a & $1(2)$ & 1.00 & $2-3$ \\
\hline Typhlops & 20 & $d$ & $r$ & $\mathrm{nc}$ & a & $c(i)$ & 2 & $a(p)$ & $2(1,3)$ & 1.85 & $3(0)$ \\
\hline
\end{tabular}


Table 2 (part 2, continued)

\begin{tabular}{|c|c|c|c|c|c|c|c|c|c|c|c|c|c|}
\hline Taxon & $10 a$ & $10 \mathrm{~b}$ & 11 & $12 a$ & $12 \mathrm{~b}$ & $13 a$ & $13 b$ & $14 a$ & $14 \mathrm{~b}$ & $15 a$ & $15 b$ & $16 a$ & $16 \mathrm{~b}$ \\
\hline Afrotyphlopinae & $18-45$ & 24.8 & $p, a$ & $216-737$ & 437 & $4-15$ & 10.8 & $125-950$ & 403 & $17-129$ & 52.4 & $33-152$ & 68.2 \\
\hline Afrotyphlops & $18-45$ & 27.2 & $\mathrm{p}(\mathrm{a})$ & $216-620$ & 391 & $4-15$ & 9.6 & $125-950$ & 481 & $17-95$ & 39.5 & $33-152$ & 60.8 \\
\hline Letheobia & $18-30$ & 22.3 & $p, a$ & $250-737$ & 484 & $8-15$ & 11.1 & $135-670$ & 350 & $35-129$ & 66.6 & $46-143$ & 79.0 \\
\hline Rhinotyphlops & $22-34$ & 25.0 & $p, a$ & $311-586$ & 425 & $14-15$ & 14.5 & $220-455$ & 323 & $27-77$ & 48.9 & $50-79$ & 65.0 \\
\hline Asiatyphlopinae & $16-36$ & 21.8 & $p, a$ & $206-750$ & 408 & $5-36$ & 14.6 & $91-750$ & 293 & $18-134$ & 49.8 & $13-133$ & 47.1 \\
\hline Acutotyphlops & $26-36$ & 30.4 & $p$ & $334-542$ & 415 & $11-31$ & 20.9 & $333-487$ & 392 & $18-58$ & 38.2 & $13-100$ & 31.1 \\
\hline Anilios & $16-24$ & 20.1 & $a(p)$ & $278-750$ & 466 & $8-36$ & 15.0 & $122-750$ & 353 & $20-134$ & 55.8 & $15-112$ & 49.7 \\
\hline Asiatyphlops & $20-30$ & 23.2 & $p, a$ & $246-520$ & 339 & $5-26$ & 11.1 & $130-430$ & 243 & $26-70$ & 35.2 & $42-100$ & 65.6 \\
\hline Cyclotyphlops & 22 & 22.0 & $\mathrm{p}$ & 294 & 294 & $\mathrm{n} / \mathrm{a}$ & $\mathrm{n} / \mathrm{a}$ & 146 & 146 & 35 & 35.1 & 32.3 & 32.3 \\
\hline Grypotyphlops & $24-34$ & 29.0 & $\mathrm{p}$ & $448-526$ & 487 & $7-13$ & 10.0 & 630 & 630 & $30-66$ & 48 & $17-133$ & 75.1 \\
\hline Indotyphlops & $18-22$ & 19.4 & $a(p)$ & $229-468$ & 345 & $7-15$ & 10.8 & $91-285$ & 175 & $28-130$ & 57.6 & $22-76$ & 46.4 \\
\hline Malayotyphlops & $24-30$ & 26.8 & $\mathrm{p}$ & $300-461$ & 367 & $9-14$ & 11.9 & $122-445$ & 256 & $31-58$ & 42.0 & $28-71$ & 51.8 \\
\hline Ramphotyphlops & $18-30$ & 21.7 & $\mathrm{p}, \mathrm{a}$ & $206-653$ & 419 & $8-36$ & 19.8 & $117-480$ & 302 & $18-88$ & 48.8 & $17-70$ & 31.8 \\
\hline Sundatyphlops & 22 & 22.0 & $n / a$ & 496 & 496 & 16 & 16.0 & 395 & 395 & $33-58$ & 45.5 & $15-52$ & 33.3 \\
\hline Xerotyphlops & $20-24$ & 23.5 & $\mathrm{p}$ & $206-435$ & 355 & $10-16$ & 13.0 & $220-350$ & 292 & $37-55$ & 45.6 & 87.7 & 87.7 \\
\hline Madatyphlopinae & $20-28$ & 22.9 & $p, a$ & $252-600$ & 429 & $6-18$ & 11.2 & $176-600$ & 320 & $27-85$ & 45.6 & $33-125$ & 53.7 \\
\hline Madatyphlops & $20-28$ & 22.9 & $\mathrm{p}, \mathrm{a}$ & $252-600$ & 429 & $6-18$ & 11.2 & $176-600$ & 320 & $27-85$ & 45.6 & $33-125$ & 53.7 \\
\hline Typhlopinae & $16-24$ & 20.3 & $p, a$ & $170-629$ & 369 & $6-22$ & 11.7 & $101-522$ & 264 & $16-77$ & 42.7 & $18-270$ & 48.7 \\
\hline Amerotyphlops & $16-22$ & 19.1 & $a(p)$ & $170-556$ & 327 & $6-15$ & 9.4 & $101-522$ & 287 & $16-77$ & 38.7 & $23-270$ & 66.8 \\
\hline Antillotyphlops & $16-24$ & 20.5 & $\mathrm{p}$ & 299-499 & 378 & $11-14$ & 12.3 & $110-360$ & 233 & $37-70$ & 46.8 & $30-61$ & 44.5 \\
\hline Cubatyphlops & $20-24$ & 22.1 & $p, a$ & $351-629$ & 503 & 22 & 22.0 & $197-460$ & 304 & $36-75$ & 54.7 & $29-85$ & 50.4 \\
\hline Typhlops & $18-22$ & 20.0 & $\mathrm{p}, \mathrm{a}$ & $231-457$ & 312 & 8-19 & 13.1 & $126-445$ & 243 & $23-57$ & 35.9 & $18-88$ & 36.8 \\
\hline
\end{tabular}

Table 2 (part 3, continued)

\begin{tabular}{|c|c|c|c|c|}
\hline Taxon & 17 & 18 & 19 & 20 \\
\hline Afrotyphlopinae & $\mathrm{v}(\mathrm{wt}, \mathrm{cr}, \mathrm{bn}, \mathrm{yl}, \mathrm{bl}, \mathrm{pl}-\mathrm{gn}, \mathrm{pk})$ & $w t, c r, y l(b n, b k)$ & 1,0 & un(dl) \\
\hline Afrotyphlops & dk-bn (yl,pl-bl,pk,pl-gn) & yl,yl-wt,cr,wt & $1(0)$ & un(dl) \\
\hline Letheobia & $\mathrm{pl}(\mathrm{pk}, \mathrm{cr}, \mathrm{wt}, \mathrm{un})$ & $\mathrm{pl}(\mathrm{pk}, \mathrm{cr}, \mathrm{wt}, \mathrm{un})$ & $0(1)$ & un \\
\hline Rhinotyphlops & bn $(p l-y l, p l-g n, p k)$ & $w t, y l-w t(b n, b k)$ & 1 & un(dl) \\
\hline Asiatyphlopinae & bn,dk-bn,pl-bn(v) & wt,cr,yl-wt & $1(0)$ & $\mathrm{un}(\mathrm{dl})$ \\
\hline Acutotyphlops & dk-bn(gd-or) & $y l-w t, g d$ & 1 & un $(b r, d l)$ \\
\hline Anilios & bn(cr,tn,pk,gy,pl-yl) & wt,cr,yl-wt & $1(0)$ & un(dl) \\
\hline Asiatyphlops & bn(gd-bn,ol-dn,bk-bn,yl-bn,rd-bn,lv-gy,bk) & yl,yl-wt(wt,cr,yl-tn,yl-bn,pl-bn) & $1(0)$ & un(dl) \\
\hline Cyclotyphlops & dk-bn & bn & 1 & un \\
\hline Grypotyphlops & gy,bn,gd-bn & gy-wt,yl,pl-bn & 1 & un(dl) \\
\hline Indotyphlops & bn(cr,gy,yl-bn,rd-bn,lv-gy,bk) & v(wt,cr,pl-bn,lv-gy) & $1(0)$ & un(dl) \\
\hline Malayotyphlops & $\mathrm{v}(\mathrm{bn}, \mathrm{gy}-\mathrm{bn}, \mathrm{rd}-\mathrm{bn}, \mathrm{gn}-\mathrm{bk}, \mathrm{bk})$ & $v(c r, y l-w t, r d, p k, p k-y l)$ & 1 & un(dl,sp,nk) \\
\hline Ramphotyphlops & $\mathrm{dk}-\mathrm{bn}(\mathrm{bn}, \mathrm{tn}, \mathrm{gy}, \mathrm{lv})$ & cr,yl-wt(gy-gn,pk-wt,tn,bn) & $1(0)$ & un(dl) \\
\hline Sundatyphlops & dk-bn,ol,ol-gy,pl-yl & $w t, w t-p k, p l-y l$ & $1(0)$ & $\mathrm{dl}, \mathrm{vl}$,un \\
\hline Xerotyphlops & $w t, b n$ & $w t, c r, b n$ & $1(0)$ & un(dl) \\
\hline Madatyphlopinae & $b n(w t, p k, c r, y l, g y)$ & $\mathrm{v}(\mathrm{wt}, \mathrm{pk}, \mathrm{cr}, \mathrm{yl}, \mathrm{gy}, \mathrm{bn})$ & 1 & un(dl,nk) \\
\hline Madatyphlops & bn $(w t, p k, c r, y l, g y)$ & $\mathrm{v}(\mathrm{wt}, \mathrm{pk}, \mathrm{cr}, \mathrm{yl}, \mathrm{gy}, \mathrm{bn})$ & 1 & un(dl,nk) \\
\hline Typhlopinae & bn $(c r, t n, y l-w t, u n)$ & un(wt,cr,bn) & $1(0)$ & un(sp, dl,rt) \\
\hline Amerotyphlops & bn,yl & $w t, c r, y l$ & $1(0)$ & sp,dl,rt \\
\hline Antillotyphlops & bn,gy-bn,yl & wt,cr(bn) & $1(0)$ & $\mathrm{un}(\mathrm{dl})$ \\
\hline Cubatyphlops & bn(un,pk) & un(cr) & $1(0)$ & un \\
\hline Typhlops & bn,tn & un, wt, cr & $1(0)$ & un(dl) \\
\hline
\end{tabular}


caribbean herpetology

article

Table 3. Classification of snakes of the Family Typhlopidae.

\begin{tabular}{|c|c|}
\hline This study & Previous classification \\
\hline \multicolumn{2}{|l|}{ Afrotyphlopinae } \\
\hline Afrotyphlops angolensis (Bocage 1866) & Afrotyphlops angolensis \\
\hline Afrotyphlops anomalus (Bocage 1873) & Megatyphlops anomalus \\
\hline Afrotyphlops bibronii (Smith 1846) & Afrotyphlops bibronii \\
\hline Afrotyphlops blanfordii (Boulenger 1889) & Afrotyphlops blanfordii \\
\hline Afrotyphlops brevis (Scortecci 1929a) & Megatyphlops brevis \\
\hline Afrotyphlops calabresii (Gans \& Laurent 1965) & Typhlops calabresii \\
\hline Afrotyphlops comorensis (Boulenger 1889) & Typhlops comorensis \\
\hline Afrotyphlops congestus (Duméril \& Bibron 1844) & Afrotyphlops congestus \\
\hline Afrotyphlops cuneirostris (Peters 1879) & Typhlops cuneirostris \\
\hline Afrotyphlops decorosus (Peters 1875) & Letheobia decorosa \\
\hline Afrotyphlops elegans (Peters 1868) & Typhlops elegans \\
\hline Afrotyphlops fornasinii (Bianconi 1847) & Afrotyphlops fornasinii \\
\hline Afrotyphlops gierrai (Mocquard 1897) & Afrotyphlops gierrai \\
\hline Afrotyphlops jubanus (Broadley \& Wallach 2007) & Letheobia jubanus \\
\hline Afrotyphlops kaimosae (Loveridge 1935) & Afrotyphlops kaimosae \\
\hline Afrotyphlops liberiensis (Hallowell 1848) & Typhlops liberiensis \\
\hline Afrotyphlops lineolatus (Jan 1863) & Afrotyphlops lineolatus \\
\hline Afrotyphlops mucruso (Peters 1854) & Megatyphlops mucruso \\
\hline Afrotyphlops nanus Broadley \& Wallach, 2009 & Afrotyphlops nanus \\
\hline Afrotyphlops nigrocandidus (Broadley \& Wallach 2000) & Afrotyphlops nigrocandidus \\
\hline Afrotyphlops obtusus (Peters 1865) & Letheobia obtusa \\
\hline Afrotyphlops platyrhynchus (Sternfeld 1910) & Typhlops platyrhynchus \\
\hline Afrotyphlops punctatus (Leach 1819) & Afrotyphlops punctatus \\
\hline Afrotyphlops rondoensis (Loveridge 1942) & Afrotyphlops rondoensis \\
\hline Afrotyphlops schlegelii (Bianconi 1847) & Megatyphlops schlegelii \\
\hline Afrotyphlops schmidti (Laurent 1956) & Afrotyphlops schmidti \\
\hline Afrotyphlops steinhausi (Werner 1909b) & Afrotyphlops steinhausi \\
\hline Afrotyphlops tanganicanus (Laurent 1964) & Afrotyphlops tanganicanus \\
\hline Afrotyphlops usambaricus (Laurent 1964) & Afrotyphlops usambaricus \\
\hline Letheobia acutirostrata (Andersson 1916) & Letheobia acutirostrata \\
\hline Letheobia angeli (Guibé 1952) & Letheobia angeli \\
\hline Letheobia caeca (Duméril 1856) & Letheobia caeca \\
\hline Letheobia coecata (Jan 1863) & Typhlops coecata \\
\hline Letheobia crossii (Boulenger 1893) & Letheobia crossii \\
\hline Letheobia debilis (Joger 1990) & Letheobia debilis \\
\hline Letheobia episcopa Franzen \& Wallach, 2002 & Letheobia episcopa \\
\hline Letheobia erythraea (Scortecci 1929b) & Letheobia erythraea \\
\hline Letheobia feae (Boulenger 1906) & Letheobia feae \\
\hline Letheobia gracilis (Sternfeld 1910) & Letheobia gracilis \\
\hline Letheobia graueri (Sternfeld 1912) & Letheobia graueri \\
\hline
\end{tabular}


Letheobia kibarae (Witte 1953)

Letheobia largeni Broadley \& Wallach, 2007

Letheobia leucosticta (Boulenger 1898a)

Letheobia lumbriciformis (Peters 1874)

Letheobia manni (Loveridge 1941)

Letheobia newtoni (Bocage 1890)

Letheobia pallida Cope, 1868

Letheobia pauwelsi Wallach, 2005

Letheobia pembana Broadley \& Wallach, 2007

Letheobia praeocularis (Stejneger 1894)

Letheobia rufescens (Chabanaud 1916)

Letheobia simonii (Böttger 1879)

Letheobia somalica (Boulenger 1895a)

Letheobia stejnegeri (Loveridge 1931)

Letheobia sudanensis (Schmidt 1923)

Letheobia swahilica (Broadley \& Wallach 2007)

Letheobia toritensis (Broadley \& Wallach 2007)

Letheobia uluguruensis (Barbour \& Loveridge 1928)

Letheobia wittei (Roux-Estève 1974)

Letheobia zenkeri (Sternfeld 1908)

Rhinotyphlops ataeniatus (Boulenger 1912)

Rhinotyphlops boylei (FitzSimons 1932)

Rhinotyphlops Ialandei (Schlegel 1839)

Rhinotyphlops leucocephalus (Parker 1930)

Rhinotyphlops schinzi (Böttger 1887)

Rhinotyphlops scortecci (Gans \& Laurent 1965)

Rhinotyphlops unitaeniatus (Peters 1878)

\section{Asiatyphlopinae}

Acutotyphlops banaorum Wallach et al. 2007

Acutotyphlops infralabialis (Waite 1918a)

Acutotyphlops kunuaensis Wallach, 1995

Acutotyphlops solomonis (Parker 1939)

Acutotyphlops subocularis (Waite 1897a)

Anilios affinis (Boulenger 1889)

Anilios ammodytes (Montague et al. 1914)

Anilios aspinus (Couper et al. 1998)

Anilios australis (Gray 1845)

Anilios batillus (Waite 1894)

Anilios bicolor (Peters 1858)

Anilios bituberculatus (Peters 1863)

Anilios broomi (Boulenger 1898b)

Anilios centralis (Storr 1984)
Letheobia kibarae

Letheobia largeni

Letheobia leucosticta

Letheobia lumbriciformis

Typhlops manni

Letheobia newtoni

Letheobia pallida

Letheobia pauwelsi

Letheobia pembana

Letheobia praeocularis

Letheobia rufescens

Letheobia simonii

Letheobia somalica

Letheobia stejnegeri

Letheobia sudanensis

Letheobia swahilica

Letheobia toritensis

Letheobia uluguruensis

Letheobia wittei

Typhlops zenkeri

Letheobia ataeniatus

Rhinotyphlops boylei

Rhinotyphlops lalandei

Rhinotyphlops leucocephalus

Rhinotyphlops schinzi

Letheobia scortecci

Letheobia unitaeniata

Acutotyphlops banaorum

Acutotyphlops infralabialis

Acutotyphlops kunuaensis

Acutotyphlops solomonis

Acutotyphlops subocularis

Ramphotyphlops affinis

Ramphotyphlops ammodytes

Ramphotyphlops aspinus

Ramphotyphlops australis

Ramphotyphlops batillus

Ramphotyphlops bicolor

Ramphotyphlops bituberculatus

Ramphotyphlops broomi

Ramphotyphlops centralis 
Anilios chamodracaena (Ingram \& Covacevich 1993)

Anilios diversus (Waite 1894)

Anilios endoterus (Waite 1918b)

Anilios erycinus (Werner 1901)

Anilios ganei (Aplin 1998)

Anilios grypus (Waite 1918b)

Anilios guentheri (Peters 1865)

Anilios hamatus (Storr 1981)

Anilios howi (Storr 1983)

Anilios kimberleyensis (Storr 1981)

Anilios leptosomus (Robb 1972)

Anilios leucoproctus (Boulenger 1889)

Anilios ligatus (Peters 1879)

Anilios longissimus (Aplin 1998)

Anilios margaretae (Storr 1981)

Anilios micrommus (Storr 1981)

Anilios minimus (Kinghorn 1929)

Anilios nema (Shea \& Horner 1997)

Anilios nigrescens Gray, 1845

Anilios nigroterminatus (Parker 1931)

Anilios pilbarensis (Aplin \& Donnellan 1993)

Anilios pinguis (Waite 1897b)

Anilios proximus (Waite 1893)

Anilios robertsi (Couper et al. 1998)

Anilios silvia (Ingram \& Covacevich 1993)

Anilios splendidus (Aplin 1998)

Anilios torresianus (Schlegel 1839)

Anilios tovelli (Loveridge 1945)

Anilios troglodytes (Storr 1981)

Anilios unguirostris (Peters 1867b)

Anilios waitii (Boulenger 1895b)

Anilios wiedii (Peters 1867a)

Anilios yampiensis (Storr 1981)

Anilios yirrikalae (Kinghorn 1942)

Asiatyphlops bothriorhynchus (Günther 1864)

Asiatyphlops diardii (Schlegel 1839)

Asiatyphlops fuscus (Duméril \& Duméril 1851)

Asiatyphlops giadinhensis (Bourret 1937)

Asiatyphlops klemmeri (Taylor 1962)

Asiatyphlops koshunensis (Ôshima 1916)

Asiatyphlops leucomelas (Boulenger 1890)

Asiatyphlops muelleri (Schlegel 1839)

Asiatyphlops oatesii (Boulenger 1890)
Ramphotyphlops chamodracaena

Ramphotyphlops diversus

Ramphotyphlops endoterus

Ramphotyphlops erycinus

Ramphotyphlops ganei

Ramphotyphlops grypus

Ramphotyphlops guentheri

Ramphotyphlops hamatus

Ramphotyphlops howi

Ramphotyphlops kimberleyensis

Ramphotyphlops leptosomus

Ramphotyphlops leucoproctus

Ramphotyphlops ligatus

Ramphotyphlops longissimus

Ramphotyphlops margaretae

Ramphotyphlops micrommus

Ramphotyphlops minimus

Ramphotyphlops nema

Ramphotyphlops nigrescens

Ramphotyphlops nigroterminatus

Ramphotyphlops pilbarensis

Ramphotyphlops pinguis

Ramphotyphlops proximus

Ramphotyphlops robertsi

Ramphotyphlops silvia

Ramphotyphlops splendidus

Ramphotyphlops polygrammicus

Ramphotyphlops tovelli

Ramphotyphlops troglodytes

Ramphotyphlops unguirostris

Ramphotyphlops waitii

Ramphotyphlops wiedii

Ramphotyphlops yampiensis

Ramphotyphlops yirrikalae

Typhlops bothriorhynchus

Typhlops diardii

Typhlops fuscus

Typhlops giadinhensis

Typhlops klemmeri

Typhlops koshunensis

Typhlops leucomelas

Typhlops muelleri

Typhlops oatesii 
Asiatyphlops roxaneae (Wallach 2001)

Asiatyphlops siamensis (Günther 1864)

Asiatyphlops tenuicollis (Peters 1864)

Asiatyphlops trangensis (Taylor 1962)

Cyclotyphlops deharvengi in den Bosch \& Ineich 1994

Grypotyphlops acutus (Duméril \& Bibron 1844)

Indotyphlops ahsanai (Khan 1999)

Indotyphlops albiceps (Boulenger 1898a)

Indotyphlops braminus (Daudin 1803)

Indotyphlops exiguus (Jan 1864)

Indotyphlops filiformis (Duméril \& Bibron 1844)

Indotyphlops fletcheri (Wall 1919)

Indotyphlops hypsobothrius (Werner 1917)

Indotyphlops jerdoni (Boulenger 1890)

Indotyphlops khoratensis (Taylor 1962)

Indotyphlops Iankaensis (Taylor 1947)

Indotyphlops lazelli (Wallach \& Pauwels 2004)

Indotyphlops loveridgei (Constable 1949)

Indotyphlops madgemintonae (Khan 1999)

Indotyphlops malcolmi (Taylor 1947)

Indotyphlops meszoelyi (Wallach 1999)

Indotyphlops ozakiae (Niyomwan et al. 2001)

Indotyphlops pammeces (Günther 1864)

Indotyphlops porrectus (Stoliczka 1871)

Indotyphlops schmutzi (Auffenberg 1980)

Indotyphlops tenebrarum (Taylor 1947)

Indotyphlops veddae (Taylor 1947)

Indotyphlops violaceus (Taylor 1947)

Malayotyphlops canlaonensis (Taylor 1917)

Malayotyphlops castanotus (Wynn \& Leviton 1993)

Malayotyphlops collaris (Wynn \& Leviton 1993)

Malayotyphlops hypogius (Savage 1950)

Malayotyphlops koekkoeki (Brongersma 1934)

Malayotyphlops kraali (Doria 1874)

Malayotyphlops luzonensis (Taylor 1919)

Malayotyphlops manilae (Taylor 1919)

Malayotyphlops ruber (Böttger 1897)

Malayotyphlops ruficauda (Gray 1845)

Ramphotyphlops acuticauda (Peters 1877)

Ramphotyphlops adocetus Wynn et al. 2012

Ramphotyphlops angusticeps (Peters 1877)

Ramphotyphlops becki Tanner, 1948

Ramphotyphlops conradi (Peters 1875)
Typhlops roxaneae

Typhlops siamensis

Typhlops tenuicollis

Typhlops trangensis

Cyclotyphlops deharvengi

Letheobia acutus

Typhlops ahsanai

Ramphotyphlops albiceps

Ramphotyphlops braminus

Typhlops exiguus

Typhlops filiformis

Typhlops fletcheri

Typhlops hypsobothrius

Typhlops jerdoni

Typhlops khoratensis

Typhlops lankaensis

Typhlops lazelli

Typhlops loveridgei

Typhlops madgemintonae

Typhlops malcolmi

Typhlops meszoelyi

Ramphotyphlops ozakiae

Typhlops pammeces

Typhlops porrectus

Typhlops schmutzi

Typhlops tenebrarum

Typhlops veddae

Typhlops violaceus

Typhlops canlaonensis

Typhlops castanotus

Typhlops collaris

Typhlops hypogius

Typhlops koekkoeki

Typhlops kraali

Typhlops luzonensis

Typhlops manilae

Typhlops ruber

Typhlops ruficaudus

Ramphotyphlops acuticauda

Ramphotyphlops adocetus

Ramphotyphlops angusticeps

Ramphotyphlops becki

Typhlops conradi 
Ramphotyphlops cumingii (Gray 1845)

Ramphotyphlops depressus Peters, 1880

Ramphotyphlops exocoeti (Boulenger 1887)

Ramphotyphlops flaviventer (Peters 1864)

Ramphotyphlops hatmaliyeb Wynn et al. 2012

Ramphotyphlops lineatus (Schlegel 1839)

Ramphotyphlops lorenzi (Werner 1909b)

Ramphotyphlops mansuetus (Barbour 1921)

Ramphotyphlops marxi (Wallach 1993)

Ramphotyphlops melanocephalus (Duméril \& Bibron 1844)

Ramphotyphlops multilineatus (Schlegel 1839)

Ramphotyphlops olivaceus (Gray 1845)

Ramphotyphlops similis (Brongersma 1934)

Ramphotyphlops suluensis (Taylor 1918)

Ramphotyphlops supranasalis (Brongersma 1934)

Ramphotyphlops willeyi (Boulenger 1900)

Sundatyphlops polygrammicus (Schlegel 1839)

Xerotyphlops etheridgei (Wallach 2002)

Xerotyphlops socotranus (Boulenger 1889)

Xerotyphlops vermicularis (Merrem 1820)

Xerotyphlops wilsoni (Wall 1908)

\section{Madatyphlopinae}

Madatyphlops andasibensis (Wallach \& Glaw 2009)

Madatyphlops arenarius (Grandidier 1872)

Madatyphlops boettgeri (Boulenger 1893)

Madatyphlops decorsei (Mocquard 1901)

Madatyphlops domerguei (Roux-Estève 1980)

Madatyphlops madagascariensis (Böttger 1877)

Madatyphlops microcephalus (Werner 1909a)

Madatyphlops mucronatus (Böttger 1880)

Madatyphlops ocularis (Parker 1927)

Madatyphlops rajeryi (Renoult \& Raselimanana 2009)

Madatyphlops reuteri (Böttger 1881)

\section{Typhlopinae}

Amerotyphlops amoipira (Rodrigues \& Juncá 2002)

Amerotyphlops brongersmianus (Vanzolini 1976)

Amerotyphlops costaricensis (Jiménez \& Savage 1963)

Amerotyphlops lehneri (Roux 1926)

Amerotyphlops microstomus (Cope 1866)

Amerotyphlops minuisquamus (Dixon \& Hendricks 1979)

Amerotyphlops paucisquamus (Dixon \& Hendricks 1979)

Amerotyphlops reticulatus (Linnaeus 1758)
Ramphotyphlops cumingii

Ramphotyphlops depressus

Ramphotyphlops exocoeti

Ramphotyphlops flaviventer

Ramphotyphlops hatmaliyeb

Ramphotyphlops lineatus

Ramphotyphlops lorenzi

Ramphotyphlops mansuetus

Ramphotyphlops marxi

Ramphotyphlops melanocephalus

Ramphotyphlops multilineatus

Ramphotyphlops olivaceus

Ramphotyphlops similis

Ramphotyphlops suluensis

Ramphotyphlops supranasalis

Ramphotyphlops willeyi

Ramphotyphlops polygrammicus

Typhlops etheridgei

Typhlops socotranus

Typhlops vermicularis

Typhlops wilsoni

Typhlops andasibensis

Typhlops arenarius

Typhlops boettgeri

Typhlops decorsei

Typhlops domerguei

Typhlops madagascariensis

Typhlops microcephalus

Typhlops mucronatus

Typhlops ocularis

Typhlops rajeryi

Typhlops reuteri

Typhlops amoipira

Typhlops brongersmianus

Typhlops costaricensis

Typhlops lehneri

Typhlops microstomus

Typhlops minuisquamus

Typhlops paucisquamus

Typhlops reticulatus 
Amerotyphlops stadelmani (Schmidt 1936)

Amerotyphlops tasymicris (Thomas 1974a)

Amerotyphlops tenuis (Salvin 1860)

Amerotyphlops trinitatus (Richmond 1965)

Amerotyphlops tycherus (Townsend et al. 2008)

Amerotyphlops yonenagae (Rodrigues 1991)

Antillotyphlops annae (Breuil 1999)

Antillotyphlops catapontus (Thomas 1966)

Antillotyphlops dominicanus (Stejneger 1904)

Antillotyphlops geotomus (Thomas 1966)

Antillotyphlops granti (Ruthven \& Gaige 1935)

Antillotyphlops guadeloupensis (Richmond 1966)

Antillotyphlops hypomethes (Hedges \& Thomas 1991)

Antillotyphlops monastus (Thomas 1966)

Antillotyphlops monensis (Schmidt 1926)

Antillotyphlops naugus (Thomas 1966)

Antillotyphlops platycephalus (Duméril \& Bibron 1844)

Antillotyphlops richardi (Duméril \& Bibron 1844)

Cubatyphlops anchaurus (Thomas \& Hedges 2007)

Cubatyphlops anousius (Thomas \& Hedges 2007)

Cubatyphlops arator (Thomas \& Hedges 2007)

Cubatyphlops biminiensis (Richmond 1955)

Cubatyphlops caymanensis (Sackett 1940)

Cubatyphlops contorhinus (Thomas \& Hedges 2007)

Cubatyphlops epactius (Thomas 1968)

Cubatyphlops golyathi (Dominguez \& Moreno 2009)

Cubatyphlops notorachius (Thomas \& Hedges 2007)

Cubatyphlops paradoxus (Günther 1875)

Cubatyphlops perimychus (Thomas \& Hedges 2007)

Cubatyphlops satelles (Thomas \& Hedges 2007)

Typhlops agoralionis Thomas \& Hedges 2007

Typhlops capitulatus Richmond 1964

Typhlops eperopeus Thomas \& Hedges 2007

Typhlops gonavensis Richmond 1964

Typhlops hectus Thomas 1974b

Typhlops jamaicensis (Shaw 1802)

Typhlops leptolepis Dominguez et al. 2013

Typhlops lumbricalis (Linnaeus 1758)

Typhlops oxyrhinus Dominguez \& Diaz Jr. 2011

Typhlops pachyrhinus Dominguez \& Diaz Jr. 2011

Typhlops proancylops Thomas \& Hedges 2007

Typhlops pusillus Barbour 1914

Typhlops rostellatus Stejneger 1904
Typhlops stadelmani

Typhlops tasymicris

Typhlops tenuis

Typhlops trinitatus

Typhlops tycherus

Typhlops yonenagae

Typhlops annae

Typhlops catapontus

Typhlops dominicanus

Typhlops geotomus

Typhlops granti

Typhlops guadeloupensis

Typhlops hypomethes

Typhlops monastus

Typhlops monensis

Typhlops naugus

Typhlops platycephalus

Typhlops richardi

Typhlops anchaurus

Typhlops anousius

Typhlops arator

Typhlops biminiensis

Typhlops caymanensis

Typhlops contorhinus

Typhlops epactius

Typhlops golyathi

Typhlops notorachius

Typhlops paradoxus

Typhlops perimychus

Typhlops satelles

Typhlops agoralionis

Typhlops capitulatus

Typhlops eperopeus

Typhlops gonavensis

Typhlops hectus

Typhlops jamaicensis

Typhlops leptolepis

Typhlops lumbricalis

Typhlops oxyrhinus

Typhlops pachyrhinus

Typhlops proancylops

Typhlops pusillus

Typhlops rostellatus 
Typhlops schwartzi Thomas 1989a

Typhlops silus Legler 1959

Typhlops sulcatus Cope 1868a

Typhlops sylleptor Thomas \& Hedges 2007

Typhlops syntherus Thomas 1965

Typhlops tetrathyreus Thomas 1989

Typhlops titanops Thomas 1989
Typhlops schwartzi

Typhlops lumbricalis

Typhlops sulcatus

Typhlops sylleptor

Typhlops syntherus

Typhlops tetrathyreus

Typhlops titanops

separation (narrow, moderate, and wide) is often reported but it has little diagnostic value with regard to clades identified here in the molecular phylogenies. Likewise, while we found retrocloacal sacs and a protrusible hemipenis (Robb 1966) to be useful in diagnosing some clades, we did not find that traits derived from viscera (Broadley \&Wallach 2007; 2009) show strong correlations with the molecular clades and therefore do not include them here. This lack of correlation between molecular phylogeny and visceral traits was also encountered in leptotyphlopids (Adalsteinsson et al. 2009). As discussed elsewhere (Hedges 2008), body shape and organ size in animals, especially snakes, is negatively allometric (i.e., disproportionate with body size) and this might explain why variation in position, shape, and gain and loss of organs does not appear to be strongly tied to phylogeny.

We identified 20 characters that we considered useful in the diagnoses. We list them by individual species (Table 1) and by subfamilies and genera (Table 2). (1) Eye (distinct, indistinct). The eye was scored as distinct if it was visible at all, and indistinct if it was indistinguishable (sometimes scored from illustrations). (2) Snout (rounded, acuminate, or beaked). Head shape in blindsnakes varies depending on the observation angle (dorsal or lateral), and whether the snout protrudes beyond the lower jaw. Our interest here was in scoring three conditions used to diagnose clades previously: a beaked condition (hooked or keeled rostral), acute condition (pointed, viewed dorsally), or the common "rounded" condition (including most other conditions such as blunt and trilobed). (3) Head scales (circular, non-circular). A circular arrangement is found in Cyclotyphlops but not in other genera. (4) Frontorostral (present, absent). This scale is present in Acutotyphlops but absent in other genera. (5) Nasal division (complete, incomplete). The nasal suture is either in contact with the rostral (complete) or not in contact (incomplete). (6) Nasal suture origin. Typically the nasal suture is in contact with a supralabial scale, or sometimes the rostral scale or the preocular scale or the seam between two supralabials or between a supralabial and the preocular. (7) Suboculars or subpreoculars (present, absent). Here we score whether there are unusual lateral head scales (suboculars, subpreoculars) besides the typical ones, which are the nasal, preocular, ocular, and labials. (8) Postoculars. The number of these scales varies among genera and is diagnostic. (9) Preocular-labial contact. The lower edge of the preocular scale typically contacts one or several supralabial scales. (10) Midbody scale rows. We summarize the ranges by taxon, and averages of species averages (or, if not available, range midpoints). (11) Scale row reduction (present, absent). If present, then the posterior scale row count is lower than the midbody (or anterior) count. This character remains unreported in many species. (12). Total scale rows (between frontal and caudal spine). We summarize the ranges by taxon, and averages (or, if not available, range midpoints). (13). Caudals. We summarize the ranges by taxon, and averages (or, if not available, range midpoints). (14) Total length $(\mathrm{mm})$. Because snakes have indeterminate growth and most measured specimens have not been sexed to determine if they are adults, we only used maximum known length within a species. For genera and subfamilies we report ranges of species maximums, and taxon averages. Historically, total length rather than snout-vent length is used for body size in the scolecophidian literature because tails are typically only $1-3 \%$ of body length. (15) Total length divided by midbody diameter. This is a commonly used ratio in scolecophidians to indicate body shape. (16) Total length divided by tail length. This is a commonly used ratio in scolecophidians to indicate relative tail length. (17) Dorsal color and (18) ventral color. For these two characters we emphasize ground color. A snake can also be unpigmented, in which case it may appear pale pink or gray. (19) Bicolor condition (present, absent). Most typhlopids are darker dorsally, which is called bicolor, although one genus (Letheobia) and occasional species 
of other genera are unicolor. We did not consider anterior-posterior color differences in this character. (20) Pattern. Most blindsnakes lack a distinct pattern, but if a pattern is present at all it is often in the form of dark lines that are 1-scale wide. Some species have pale collars, and others have blotches, but these patterns are rare and were not diagnostic of molecular clades or correlated with scalation. If the pattern has a distinctive color, we so indicate.

Here we treat the 257 described species of snakes in the Family Typhlopidae. Previously, Vidal et al. (2010) defined two related families occurring in the Old World: Gerrhopilidae (15 species) and Xenotyphlopidae ( 2 species). Since then, Xenotyphlops mocquardi (Wallach et al. 2007b) has been placed in the synonymy of X. grandidieri by Wegener et al. (2013). Members of Gerrhopilidae (and the genus Gerrhopilus) have gland-like structures peppered over the scales of the head, 1 subpreocular or subocular scale (absent in 6 species), low midbody scale rows (18-24, but usually 18; average 18.6), small body size (average, $167 \mathrm{~mm} \mathrm{TL}$ ), and typically are thin-bodied with long tails. We tentatively assign one additional species, Gerrhopilus thurstoni, to Gerrhopilidae. It is small, has low midbody scale rows (20), and has an unusual yellow dorsum similar to another Indian species of the genus, G. tindalli.

\section{Systematic accounts}

\section{Family Typhlopidae Merrem, 1820}

\section{Subfamily Afrotyphlopinae subfam. nov.}

African Blindsnakes

Type genus. Afrotyphlops Broadley \& Wallach 2009.

Diagnosis. Members of this subfamily have (1) eye, distinct or indistinct, (2) snout, rounded or beaked (rarely acuminate), (3) head scale arrangement, non-circular, (4) frontorostral, absent, (5) nasal, completely or incompletely divided, (6) nasal suture origin, 1 st or 2 nd supralabial (rarely rostral or 1 st/2nd suture), (7) subocular, absent or present, (8) postoculars, 2-6 (rarely 7; average, 3.68), (9) preocular-labial contact, supralabials 2 \& 3 (rarely none, 1st, or 4th), (10) midbody scale rows, 18-45 (average, 24.8), (11) scale row reduction, present or absent, (12) total scale rows, 216-737 (average, 437), (13) caudals, 4-15 (average, 10.8), (14) maximum total length, 125950 (average, 403) mm, (15) total length/midbody diameter, 17-129 (average, 52.4), (16) total length/tail length, 33-152 (average, 68.2), (17) dorsal color, variable (white, cream, pink, brown, yellow, pale blue, pale green, or pale red), (18) ventral color, usually white, cream, or yellow (rarely brown or black), (19) dorsum darker than, or similar to, venter, (20) overall, usually no distinctive pattern, although sometimes with longitudinal lines (Tables 1-2); molecular phylogenetic support (Fig. 1).

The subfamily Afrotyphlopinae is distinguished from the other three subfamilies by more postocular scales (3.68 versus 2.19 in Asiatyphlopinae, 2.27 in Madatyphlopinae, and 1.66 in Typhlopinae; average of species mean values), more midbody scales (24.8 versus 21.8 in Asiatyphlopinae, 22.9 in Madatyphlopinae, and 20.3 in Typhlopinae), larger maximum body size (403 mm TL versus $294 \mathrm{~mm}$ in Asiatyphlopinae, $320 \mathrm{~mm}$ in Madatyphlopinae, and $264 \mathrm{~mm}$ in Typhlopinae), and shorter tails (TL/TA 68.2 versus 47.1 in Asiatyphlopinae, 53.7 in Madatyphlopinae, and 48.7 in Typhlopinae).

Content. Three genera: Afrotyphlops Broadley \& Wallach 2009, Letheobia Cope 1868, and Rhinotyphlops Fitzinger 1843 (Table 3).

Distribution. Afrotyphlopinae is distributed almost exclusively in sub-Saharan Africa (including satellite islands and islets), with two species occurring in the Middle East.

Etymology. As for the type-genus.

Remarks. The subfamily Afrotyphlopinae is well-defined in the molecular phylogeny of Vidal et al. (2010), and in the tree here (Fig. 1). Within this African clade of blindsnakes, Vidal et al. (2010) found that the arrangement of species did not agree in some respects with the taxonomic proposal of Broadley and Wallach (2009), who 


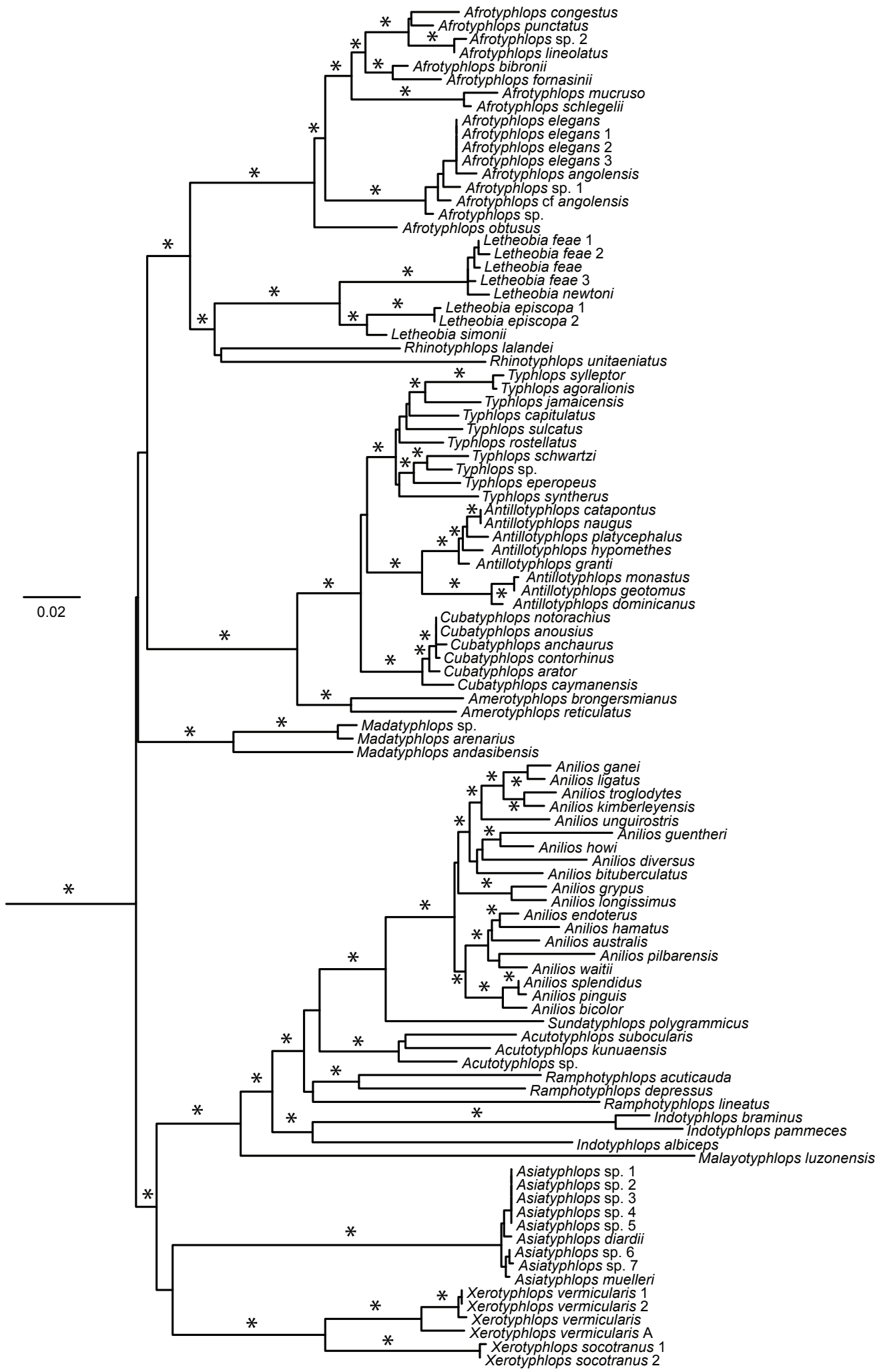

Figure 1. Phylogenetic ML tree of typhlopid snakes based on an analysis of DNA sequences of five nuclear protein-coding genes (data set A). Nodes with asterisks are supported by posterior probability $>95 \%$ and ML bootstrap probability $>70 \%$. The tree is rooted with 22 outgroup species (not shown) including a monitor lizard, and species of alethinophidian, leptotyphlopid, gerrhopilid, and xenotyphlopid snakes (Vidal et al. 2010). 
had erected two new genera: Afrotyphlops and Megatyphlops. For example, species assigned by those authors to Megatyphlops appeared nested among species assigned by them to Afrotyphlops. As has been noted (Broadley \& Wallach 2009), there has been a great diversity of different generic assignments for African species of typhlopid snakes. Here, we have added new sequences to the molecular phylogeny and have interpreted these new results in light of our compilation of morphological data (Tables 1-2). In so doing, we have corrected the errors in the current taxonomy of this group (subfamily), reassigning some species of Letheobia, Rhinotyphlops, and Typhlops to other genera. We believe that this new taxonomy retains the status quo as best as possible and recognizes major clades of species sharing suites of morphological traits.

\section{Genus Afrotyphlops Broadley \& Wallach, 2009}

African Giant Blindsnakes

Type species. Acontias punctatus Leach in Bowdich 1819:493, by original designation.

Diagnosis. Species of Afrotyphlops have (1) eye, distinct, (2) snout, rounded (sometimes beaked or acuminate), (3) head scale arrangement, non-circular, (4) frontorostral, absent, (5) nasal, incompletely divided (rarely complete), (6) nasal suture origin, 1st supralabial (sometimes 2 nd supralabial or rarely rostral), (7) suboculars or subpreoculars, absent (rarely a subpreocular/intercalary present), (8) postoculars, 2-4 (rarely 5-7; average 3.70), (9) preocular-labial contact, supralabials 2 \& 3 (rarely none, 1-2, 1-3, or 2), (10) midbody scale rows, 18-45 (average, 27.2), (11) scale row reduction, present (rarely absent), (12) total scale rows, 216-620 (average, 391), (13) caudals, 4-15 (average, 9.6), (14) maximum total length, 125-950 (average, 481) mm, (15) total length/midbody diameter, 17-95 (average, 39.5), (16) total length/tail length, 33-152 (average, 60.8), (17) dorsal color, dark brown (sometimes yellow, bluish, reddish, or greenish), (18) ventral color, yellow, yellowish, cream, or white, (19) dorsum darker than venter, (20) overall, usually patternless, but sometimes with longitudinal lines (Tables 1-2); molecular phylogenetic support (Fig. 1).

From Letheobia, Afrotyphlops is distinguished by having a distinct eye (versus indistinct), lacking subocular scales (versus present), and having a dark (versus pale) dorsum. Species of Afrotyphlops also average larger in size and have more robust bodies, although there is variation in those traits. From Rhinotyphlops, Afrotyphlops is distinguished by its more rounded snout in lateral profile (versus beaked), except several species formerly in the genus Megatyphlops, fewer caudals (9.6 versus 14.5), and larger size (481 versus $323 \mathrm{~mm}$ average TL).

Content. Twenty-nine species: Afrotyphlops angolensis, A. anomalus, A. bibronii, A. blanfordii, A. brevis, $A$. calabresii, A. comorensis, A. congestus, A. cuneirostris, A. decorosus, A. elegans, A. fornasinii, A. gierrai, A. jubanus, A. kaimosae, A. liberiensis, A. lineolatus, A. mucruso, A. nanus, A. nigrocandidus, A. obtusus, A. platyrhynchus, A. punctatus, A. rondoensis, A. schlegelii, A. schmidti, A. steinhausi, A. tanganicanus, and A. usambaricus (Table 3).

Distribution. Afrotyphlops is distributed entirely in sub-Saharan Africa (including satellite islands and islets).

Etymology. The generic name is a masculine noun formed from the adjective africanus ( $a$, um; i.e.,' from Africa') and the Greek noun typhlops (the blind).

Remarks. The molecular phylogeny of Vidal et al. (2010), and the updated phylogeny here (Fig. 1), formed a group of 10 African species that includes the type species of Afrotyphlops, A. punctatus. That species and the other nine species all lack subocular scales and their nasal suture origin is at the 1st supralabial, in contrast to most species of Letheobia that have one or more suboculars and a nasal suture origin at the 2 nd supralabial. Other characters (see diagnosis above) also were consistent with the division of the two genera. We include in this genus most species of Afrotyphlops listed by Broadley and Wallach (2009), but not all. They included A. angeli and $A$. manni, but we place them here in Letheobia based on the diagnostic characters (e.g., indistinct eye, presence of subocular, and nasal suture origin at the 2 nd supralabial). Also, of the 10 species of Afrotyphlops with molecular data, two (A. mucroso and A. schlegelii) were placed in a separate genus, Megatyphlops, by Broadley and Wallach (2009). However, their position in the tree, nested within the clade of Afrotyphlops species (Vidal et al. 2010), indicated that either Megatyphlops is a synonym of Afrotyphlops, or that additional genera should be recognized to 
correct the paraphyly of Afrotyphlops. Because it is not obvious to us how character data would justify subdividing Afrotyphlops into many genera, we instead place Megatyphlops in the synonymy of Afrotyphlops. In addition, the molecular phylogenies show that a species placed in the genus Letheobia (L. obtusa) by Broadley and Wallach clustered with the Afrotyphlops clade. Therefore, we have transferred that species and its close relatives (L. decorosa and L. jubana) to Afrotyphlops and note that their external characters agree with our diagnosis of Afrotyphlops: all 3 species lack a subocular scale and 2 of those species have the nasal suture origin at the 1st supralabial (1st or 2nd in A. jubanus). Finally, we place four species of Typhlops in Afrotyphlops: T. calabresii, T. cuneirostris, T. comorensis, and T. platyrhynchus. These species have flattened snouts and are reddish dorsally, suggesting that they are related, and they share diagnostic characters of Afrotyphlops: distinct eye, nasal incompletely divided, subocular absent, robust body, and a dark dorsum.

\section{Genus Letheobia Cope, 1868}

African Gracile Blindsnakes

Type species. Onychocephalus caecus Duméril, 1856:462, by subsequent designation of Peters, 1881:70.

Diagnosis. Species of Letheobia have (1) eye, indistinct, (2) snout, beaked or rounded, (3) head scale arrangement, non-circular, (4) frontorostral, absent, (5) nasal, completely or incompletely divided, (6) nasal suture origin, 2nd supralabial (rarely, 1st supralabial, suture (1/2), rostral, or preocular), (7) suboculars or subpreoculars, present (rarely absent), (8) postoculars, 3-4 (rarely 2, 5-7; average, 3.55), (9) preocular-labial contact, supralabials $2 \& 3$ (sometimes 2 nd, 3 rd, 2-4, or none), (10) midbody scale rows, 18-30 (average 22.3), (11) scale row reduction, present or absent, (12) total scale rows, 250-737 (average, 484), (13) caudals, 8-15 (average, 11.1), (14) maximum total length, 135-670 (average, 350) mm, (15) total length/midbody diameter, 35-129 (average, 66.6), (16) total length/tail length, 45.5-143 (average, 79.0), (17) dorsal color, usually pale (pink, cream, white, colorless), (18) ventral color, usually pale (pink, cream, white, unpigmented), (19) dorsum same color as venter, and (20) overall, a patternless, unicolor snake (Tables 1-2); molecular phylogenetic support (Fig. 1).

From Afrotyphlops and Rhinotyphlops, Letheobia is distinguished by having an indistinct eye (versus distinct), having subocular scales present (versus absent), and having a pale (versus dark) dorsum.

Content. Thirty-one species: Letheobia angeli, L. acutirostrata, L. caeca, L. coecata, L. crossii, L. debilis, L. episcopa, L. erythraea, L. feae, L. gracilis, L. graueri, L. kibarae, L. Iargeni, L. leucosticta, L. lumbriciformis, L. manni, L. newtoni, L. pallida, L. pauwelsi, L. pembana, L. praeocularis, L. rufescens, L. simonii, L. somalica, L. stejnegeri, L. sudanensis, L. swahilica, L. toritensis, L. uluguruensis, L. wittei, and L. zenkeri (Table 3).

Distribution. Letheobia is distributed throughout sub-Saharan Africa, including West Africa, Central Africa, and Eastern Africa, as far south as the Democratic Republic of the Congo and Tanzania.

Etymology. Although not stated in the original description, the generic name is a feminine noun formed from the Greek lethe (forgotten) and bios (life), meaning forgotten life, an appropriate reference to blindsnakes.

Remarks. The molecular phylogeny of Vidal et al. (2010), and the updated phylogeny here (Fig. 1), formed a group of four African species, placed in Letheobia by Broadley \& Wallach (2007): L. episcopa, L. feae, L. newtoni, and L. simonii. Broadley \& Wallach (2009) removed two species (L. angeli and L. manni) and placed them in Afrotyphlops, but we place them back in Letheobia here because they have the diagnostic external characters of that genus. We also tentatively place Typhlops coecatus and T. zenkeri in Letheobia based on their external morphology: L. coecata has an origin of the nasal suture at the 2 nd supralabial as in most species and usually has a pallid coloration, traits more often associated with Letheobia. Letheobia zenkeri has a subocular scale and pale, unicolor pattern, as in most Letheobia. In addition, our molecular phylogenies show that a species placed in the genus Letheobia (L. obtusa) by Broadley \& Wallach (2007) clustered with the Afrotyphlops clade. Therefore, we have transferred that species and its close relatives (L. decorosa and L. jubanus) to Afrotyphlops and note that their external characters agree with our diagnosis of Afrotyphlops: all three species lack a subocular scale and two of those species have the nasal suture origin at the 1st supralabial (1st or 2nd in A. jubanus). Broadley \& Wallach (2007) clarified the type species desig- 
nation of this genus.

\section{Genus Rhinotyphlops Fitzinger, 1843}

African Beaked Blindsnakes

Type species. Typhlops lalandei Schlegel, 1839:38, by original designation.

Diagnosis. Species of Rhinotyphlops have (1) eye, distinct, (2) snout, beaked (rarely rounded), (3) head scale arrangement, non-circular, (4) frontorostral, absent, (5) nasal, completely or incompletely divided, (6) nasal suture origin, 2nd supralabial (sometimes 1st supralabial), (7) suboculars or subpreoculars, absent (sometimes present), (8) postoculars, 4-6 (rarely 2-3; average 4.21), (9) preocular-labial contact, supralabials 2 \& 3 (sometimes 2nd or 2-4), (10) midbody scale rows, 22-34 (average, 25.0), (11) scale row reduction, present (sometimes absent), (12) total scale rows, 311-586 (average, 425), (13) caudals, 14-15 (average, 14.5), (14) maximum total length, 220455 (average, 323) mm, (15) total length/midbody diameter, 27-77 (average, 48.9), (16) total length/tail length, 50.2-78.5 (average, 65.0), (17) dorsal color, brown (sometimes yellowish, greenish, or reddish), (18) ventral color, white or yellowish (sometimes brownish or black), (19) dorsum darker than venter, (20) overall, either patternless or with longitudinal lines (Tables 1-2); molecular phylogenetic support (Fig. 1).

From Letheobia, Rhinotyphlops is distinguished by having a distinct eye (versus indistinct), lacking subocular scales (versus present), having a higher average number of midbody scale rows (25.0 versus 22.3), having a higher average number of caudals (14.5 versus 11.1), and having a dark (versus pale) dorsum. From Afrotyphlops, Rhinotyphlops is distinguished by having more caudals (14.5 versus 9.6) and smaller size (323 versus 481 mm average TL).

Content. Seven species: Rhinotyphlops ataeniatus, R. scortecci, R. unitaeniatus, R. boylei, R. Ialandei, R. leucocephalus, and R. schinzi (Table 3).

Distribution. Rhinotyphlops is distributed in eastern and southern Africa to South Africa. Four of the species occur in Somalia.

Etymology. The generic name is a masculine noun formed from the Greek adjective rhinos (beaked) and Greek noun typhlops (the blind), in reference to the beak-shaped snout of these blindsnakes.

Remarks. The molecular phylogeny of Vidal et al. (2010), and the updated phylogeny here (Fig. 1), place the type species of Rhinotyphlops, R. lalandei, in a group with another beaked species, $R$. unitaeniatus. The latter species was placed in the genus Letheobia by Broadley \& Wallach (2007). Therefore, we have transferred that species and its close relatives in the L. unitaeniata complex (L. ataeniata and L. scorteccii) to Rhinotyphlops and note that their external characters agree with our diagnosis of Rhinotyphlops.

\section{Subfamily Asiatyphlopinae subfam. nov.}

Asian Blindsnakes

Type genus. Asiatyphlops gen. nov.

Diagnosis. Members of this subfamily have (1) eye, distinct (rarely indistinct), (2) snout, rounded (sometimes beaked or acuminate), (3) head scale arrangement, non-circular (circular in Cyclotyphlops), (4) frontorostral, absent (present in Acutotyphlops), (5) nasal, completely or incompletely divided, (6) nasal suture origin, 2nd supralabial (rarely 1st, $1 / 2$ suture, 2/preocular suture, or preocular), (7) suboculars or subpreoculars, absent or present, (8) postoculars, 1-5 (average, 2.19), (9) preocular-labial contact, supralabials $2 \& 3$ (rarely none, 3rd, or preocular absent), (10) midbody scale rows, 16-36 (average, 21.8), (11) scale row reduction, present or absent, (12) total scale rows, 206-750 (average, 408), (13) caudals, 5-36 (average, 14.6), (14) maximum total length, 91-750 (average, 293) $\mathrm{mm},(15)$ total length/midbody diameter, 18-134 (average, 49.8), (16) total length/tail length, 13-133 (average, 47.1), (17) dorsal color, variable but usually shades of brown, (18) ventral color, variable but usually white, cream, or yellowish, (19) dorsum darker than venter, (20) overall, usually no distinctive pattern (sometimes with longitudinal 
lines) (Tables 1-2); molecular phylogenetic support (Fig. 1).

The subfamily Asiatyphlopinae is distinguished from Afrotyphlopinae in having fewer postocular scales (2.19 versus 3.68; average of species mean values), fewer midbody scales (21.8 versus 24.8), smaller body size (293 versus $403 \mathrm{~mm} \mathrm{TL}$ ), and longer tails (TL/TA 47.1 versus 68.2). It is distinguished from Typhlopinae in having more postocular scales ( 2.19 versus 1.66; average of species averages), more midbody scales (21.8 versus 20.3), and slightly larger body size (294 versus $264 \mathrm{~mm} \mathrm{TL}$ ). Individual genera of Asiatyphlopinae differ from Madatyphlopinae in the following ways: Acutotyphlops has a frontorostral and complete nasal division (versus absent, and suture incomplete), Cyclotyphlops has a circular arrangement of head scales (versus non-circular), Grypotyphlops has suboculars present (versus absent), Indotyphlops has 1 postocular (versus 2-3), Asiatyphlops, Malayotyphlops, and Xerotyphlops have fewer average total scale rows (339, 367, and 355 versus 429), Ramphotyphlops and Sundatyphlops have longer tails (TL/TA = 31.8 and 33.3 versus 53.7, averages), and Anilios has a solid, awn-like protrusible hemipenis and retrocloacal sacs (versus non-protrusible hemipenis and retrocloacal sacs absent).

Content. Ten genera: Acutotyphlops Wallach 1995, Anilios Gray 1845, Asiatyphlops gen. nov., Cyclotyphlops in den Bosch \& Ineich 1994, Grypotyphlops Peters 1881, Indotyphlops gen. nov., Malayotyphlops gen. nov., Ramphotyphlops Fitzinger 1843, Sundatyphlops gen. nov., and Xerotyphlops gen. nov.

Distribution. Asiatyphlopinae is distributed widely in southern and eastern Asia, the Malay Archipelago, Australasia, and islands of the western and southern Pacific.

Etymology. As for the type-genus.

Remarks. The subfamily Asiatyphlopinae was initially defined as a clade in the molecular phylogeny of Vidal et al. (2010), but the additional species added in subsequent studies (Marin et al. 2013a; Marin et al. 2013b), and this study (Figs. 1-2), have clarified the relationships further. This is the largest subfamily of typhlopids, with 10 genera and 121 species. The large morphological data set (Tables 1-2) has helped to allocate species that are not in the molecular phylogeny, and this has led to many generic changes, including description of five new genera here. The formerly pan-tropical genus Typhlops is now restricted to Caribbean islands.

\section{Genus Acutotyphlops Wallach, 1995}

Sharp-nosed Blindsnakes

Type species. Acutotyphlops kunuaensis Wallach, 1995:141, by original designation.

Diagnosis. Species of Acutotyphlops have (1) eye, distinct, (2) snout, acuminate or rounded, (3) head scale arrangement, non-circular, (4) frontorostral, present, (5) nasal, incompletely divided, (6) nasal suture origin, 2nd supralabial (sometimes 1st), (7) suboculars or subpreoculars, present (rarely absent), (8) postoculars, 3-5 (average, 4.10), (9) preocular-labial contact, supralabials $2 \& 3$, 3rd, or none, (10) midbody scale rows, 26-36 (average, 30.4), (11) scale row reduction, present, (12) total scale rows, 334-542 (average, 415), (13) caudals, 11-31 (average, 20.9), (14) maximum total length, 333-487 (average, 392) mm, (15) total length/midbody diameter, 18-58 (average, 38.2), (16) total length/tail length, 13-100 (average, 31.1), (17) dorsal color, dark brown (rarely golden orange), (18) ventral color, yellowish or gold, (19) dorsum darker than venter, (20) overall, either patternless or with bars or longitudinal lines (Tables 1-2); molecular phylogenetic support (Fig. 1).

Acutotyphlops differs from other typhlopids in having a frontorostral scale (Wallach 1995; Wallach et al. 2007a).

Content. Five species: Acutotyphlops banaorum, A. infralabialis, A. kunuaensis, A. solomonis, and A. subocularis (Table 3).

Distribution. Acutotyphlops is distributed in the Philippines, Indonesia, Papua New Guinea, and the Solomon Islands.

Etymology. The generic name is a masculine noun formed from the Latin adjective acutus (pointed) and Greek noun typhlops (the blind), in reference to the acuminate (pointed) snouts of these blindsnakes.

Remarks. The molecular phylogeny (Fig. 1) places the type species of Acutotyphlops in a group with two 
other species (one undescribed).

\section{Genus Anilios Gray, 1845}

Australian Blindsnakes

Type species. Anilios australis Gray, 1845:145, by subsequent designation of Stejneger, 1904:683.

Diagnosis. Species of Anilios have (1) eye, distinct, (2) snout, rounded or beaked (rarely acuminate), (3) head scale arrangement, non-circular, (4) frontorostral, absent, (5) nasal, completely or incompletely divided, (6) nasal suture origin, 2nd supralabial (sometimes 1st, 1/2 suture, 2/preocular suture, or preocular), (7) suboculars or subpreoculars, absent, (8) postoculars, 2 (rarely 1, 3, or 4; average, 2.21), (9) preocular-labial contact, supralabials 2 $\& 3,(10)$ midbody scale rows, $16-24$ (average, 20.1), (11) scale row reduction, absent (rarely present), (12) total scale rows, 278-750 (average, 466), (13) caudals, 8-36 (average, 15.0), (14) maximum total length, 122-750 (average, 353) mm, (15) total length/midbody diameter, 20-134 (average, 55.8), (16) total length/tail length, 15-112 (average, 49.7), (17) dorsal color, usually brown (sometimes cream, tan, pinkish, grey, or yellowish), (18) ventral color, usually white, cream, or yellowish, (19) dorsum darker than venter, (20) overall, usually patternless or sometimes with longitudinal lines (Tables 1-2); molecular phylogenetic support (Figs. 1-2).

From other genera of Asiatyphlopinae, Anilios differs from Acutotyphlops in lacking a frontorostral and from Cyclotyphlops in having non-circular head scales (versus circular arrangement). It differs from Grypotyphlops in lacking subocular scales. It differs from Asiatyphlops, Cyclotyphlops, Malayotyphlops, Indotyphlops, and Xerotyphlops in having more total scale rows (466 versus 294-367, averages). It differs from other genera except Indotyphlops in having a thin body (TL/MBD = 55.8 versus $35.1-46.6$ in those other genera; averages). It differs from Sundatyphlops and Ramphotyphlops in having relatively short tails (TL/TA $=49.7$ versus 31.8-33.3).

Content. Forty-three species: Anilios affinis, A. ammodytes, A. aspinus, A. australis, A. batillus, A. bicolor, A. bituberculatus, A. broomi, A. centralis, A. chamodracaena, A. diversus, A. endoterus, A. erycinus, A. ganei, A. grypus, A. guentheri, A. hamatus, A. howi, A. kimberleyensis, A. leptosomus, A. leucoproctus, A. ligatus, A. longissimus, A. margaretae, A. micrommus, A. minimus, A. nema, A. nigrescens, A. nigroterminatus, A. pilbarensis, A. pinguis, A. proximus, $A$. robertsi, A. silvia, A. splendidus, A. torresianus, A. tovelli, A. troglodytes, A. unguirostris, A. waitii, A. wiedii, A. yampiensis, and A. yirrikalae (Table 3). Broad molecular sampling of populations within Australia has shown that the true species diversity is even greater, likely exceeding 100 species (Marin et al. 2013b) (Fig. 2).

Distribution. Anilios is distributed almost entirely in Australia, with two species occurring additionally in New Guinea (A. leucoproctus and A. torresianus) and a third species occurring only in New Guinea (A. erycinus).

Etymology. The generic name is a masculine noun formed from the Greek an-(not) and noun helios (sun), in reference to the below-ground lifestyle of these snakes.

Remarks. Wallach (2006) named Austrotyphlops for this Australian clade, but Anilios is an earlier, available name. To diagnose the clade, Wallach (2006) used two reproductive traits that had been discussed in the literature previously (Robb 1966; McDowell 1974), for the more inclusive clade of Ramphotyphlops s.l.: a solid, awn-like protrusible hemipenis and retrocloacal sacs. Therefore, those characters are not diagnostic of the Australian clade (here, Anilios), as pointed out recently (Savage \& Boundy 2012). Also, Savage \& Boundy (2012) clarified the type species of Anilios. The current molecular phylogenies (Figs. 1-2) contain 27 of the 44 described species, which is a relatively large percentage of the genus, and those 27 species may actually represent 56-92 species based on molecular data (Marin et al. 2013a; Marin et al. 2013b). Our molecular phylogenies (Figs. 1-2) indicate that Anilios polygrammicus is a polyphyletic species, with the Australian populations (Queensland) belonging to Anilios and the Lesser Sunda Islands populations (including the type locality on Timor) belonging to another genus, Sundatyphlops (see below). With S. polygrammicus removed from Anilios, the only available name for the populations from northeastern Australia is Anilios torresianus (type locality, Murray Island, Queensland). We also assign populations from nearby southern New Guinea to this species. We suspect that further study will reveal that both Sundatyphlops polygrammicus and Anilios torresianus are complexes of species, based on their distribution on multiple 


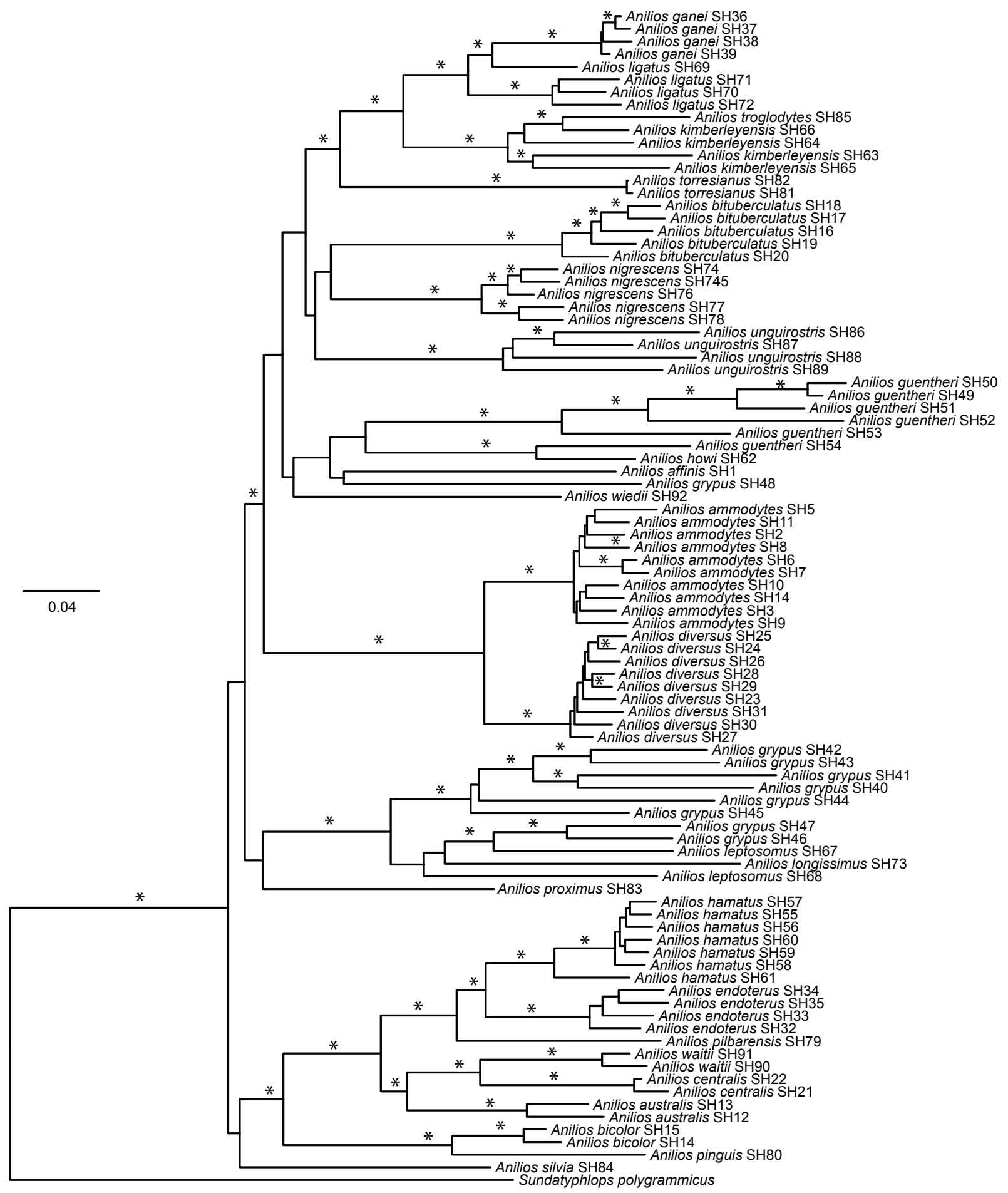

Figure 2. Phylogenetic ML tree of typhlopid snakes from Australia and nearby areas based on an analysis of DNA sequences of 3 nuclear protein-coding genes and 3 mitochondrial genes (data set B). Nodes with asterisks are supported by posterior probability $>95 \%$ and $M L$ bootstrap probability $>70 \%$. The tree is rooted with Acutotyphlops subocularis (not shown). 
land masses.

\section{Genus Asiatyphlops gen. nov.}

Southeast Asian Blindsnakes

Type species. Typhlops mülleri Schlegel, 1839:39.

Diagnosis. Species of Asiatyphlops have (1) eye, distinct (rarely indistinct), (2) snout, rounded, (3) head scale arrangement, non-circular, (4) frontorostral, absent, (5) nasal, incompletely divided (sometimes completely divided), (6) nasal suture origin, 2nd supralabial, (7) suboculars or subpreoculars, absent, (8) postoculars, 2-3 (rarely, 4-5; average, 2.69), (9) preocular-labial contact, supralabials $2 \& 3$ (sometimes 3rd only), (10) midbody scale rows, 20-30 (average, 23.2), (11) scale row reduction, present (sometimes absent), (12) total scale rows, 246-520 (average, 339), (13) caudals, 5-26 (average, 11.1), (14) maximum total length, 130-430 (average, 243) mm, (15) total length/midbody diameter, 26-70 (average, 35.2), (16) total length/tail length, 42-100 (average, 65.6), (17) dorsal color, shades of brown (golden-brown, olive-brown, blackish-brown, yellowish-brown, grayish-olive, or blue), (18) ventral color, nearly always yellow or yellowish (white, cream, yellow, yellowish-tan, yellowish-brown, bright yellow, or pale brown), (19) dorsum darker than venter, (20) overall, uniform, but sometimes with a dark lineate pattern (Tables 1-2); molecular phylogenetic support (Fig. 1).

From other genera of Asiatyphlopinae, Asiatyphlops differs from Acutotyphlops in lacking a frontorostral and from Cyclotyphlops in having non-circular head scales (versus circular arrangement). It differs from Grypotyphlops in lacking subocular scales. It differs from Acutotyphlops, Malayotyphlops, and Grypotyphlops in having fewer average midbody scale rows (23.2 versus 26.8-30.4). It differs from Anilios, Grypotyphlops, and Sundatyphlops in having fewer total scale rows (339 versus 466-496, averages). It differs from Anilios, Grypotyphlops, Indotyphlops, Ramphotyphlops, Sundatyphlops, and Xerotyphlops in having a robust body (TL/MBD $=35.2$ versus 45.5-57.6 in those other genera; averages). It differs from most genera except Grypotyphlops and Xerotyphlops in having a relatively short tail ( $\mathrm{TL} / \mathrm{TA}=65.6$ versus 31.5-51.0; averages). Compared with Indotyphlops, species of Asiatyphlops are larger (average of maximum TLs among species, 243 versus $175 \mathrm{~mm}$ ), thicker-bodied (TL/MBD = 35.2 versus 57.6, averages), shorter-tailed (TL/TA $=65.6$ versus 46.4 , averages), always have $>1$ postocular scale (versus usually 1 ), and have more midbody scale rows (average, 23.2 versus 19.4). Also, they nearly always have yellow on the venter (versus lacking a yellowish venter in Indotyphlops, except one species).

Content. Thirteen species: Asiatyphlops bothriorhynchus, A. diardii, A. fuscus, A. giadinhensis, A. klemmeri, A. koshunensis, A. leucomelas, A. muelleri, A. oatesii, A. roxaneae, A. siamensis, A. tenuicollis, and A. trangensis (Table 3).

Distribution. Asiatyphlops is broadly distributed in southern and southeastern Asia, including Bangladesh, Cambodia, southern China, India, Indonesia, Laos, Malaysia, Myanmar, Nepal, Singapore, Taiwan, Thailand, and Vietnam. Unlike Indotyphlops, it does not have a major presence in peninsular India or Sri Lanka.

Etymology. The generic name is a masculine noun formed from the adjective asianus ( $a$, um; i.e., 'from Asia') and the Greek noun typhlops (the blind).

Remarks. Blindsnakes from southern and southeast Asia have been difficult to classify in the past using morphology (Taylor 1965; McDowell 1974; Khan 1999; Wallach 1999; 2000; Wallach \& Pauwels 2004). The molecular phylogenetic evidence (Vidal et al. 2010; Marin et al. 2013a; Marin et al. 2013b) (Figs 1, 2) has assisted greatly, even though species sampling is still sparse. Those molecular data show that three major groups are present on the mainland of southern and southeast Asia: Gerrhopilidae (Vidal et al. 2010), Asiatyphlops, and Indotyphlops. Snakes of the genus Asiatyphlops are stout-bodied with 1 postocular scale, high midbody scale rows (usually $>20$ ), short tails, and yellow venters. Indotyphlops are thin-bodied blindsnakes with $>1$ postocular scale, low midbody scale rows (usually 18-20), longer tails, and venters that are pale but not yellow. Geographically, members of Asiatyphlops are primarily distributed in Southeast Asia as far west as northeastern India (Assam) and with one species ( $A$. leucomelas) occurring in Sri Lanka. Other species in Sri Lanka and peninsular India are allocated to Indotyphlops, and therefore we have some reservation in the generic assignment of $A$. leucomelas. However, the species is con- 
sistent with the diagnostic characters of Asiatyphlops. We chose Typhlops mülleri Schlegel as type species because it is in our molecular phylogeny and has a precise type locality (Padang, Sumatra).

\section{Genus Cyclotyphlops in den Bosch \& Ineich, 1994}

Sulawesi Blindsnakes

Type species. Cyclotyphlops deharvengi in den Bosch \& Ineich, 1994: 208, by monotypy.

Diagnosis. The single species of Cyclotyphlops has (1) eye, distinct, (2) snout, rounded, (3) head scale arrangement, circular, (4) frontorostral, absent, (5) nasal, completely divided, (6) nasal suture origin, junction of 1st and 2nd supralabial, (7) suboculars or subpreoculars, present, (8) postoculars, 1, (9) preocular-labial contact, absent, (10) midbody scale rows, 22, (11) scale row reduction, present, (12) total scale rows, 294, (13) caudals, unreported, (14) maximum total length, $146 \mathrm{~mm}$, (15) total length/midbody diameter, 35.1, (16) total length/tail length, 32.3, (17) dorsal color, dark brown, (18) ventral color, brown, (19) dorsum darker than venter, (20) overall, patternless (Tables 1-2); no molecular phylogenetic information is available.

Cyclotyphlops differs from all other genera in the Asiatyphlopinae in having a circular arrangement of head scales (in den Bosch \& Ineich 1994).

Content. One species: Cyclotyphlops deharvengi (Table 3).

Distribution. Cyclotyphlops is distributed on the Indonesian island of Sulawesi.

Etymology. The generic name is a masculine noun formed from the Greek nouns kyklos (circle) and typhlops (the blind), in reference to the circular arrangement of head scales in this species.

Remarks. Although this genus stands out in terms of head scalation, the same can be said of Acutotyphlops, yet the latter genus is nested deeply within Asiatyphlopinae. For that reason, there is no justification yet to recognize Cyclotyphlops as a separate family. However, until more material becomes available, including DNA sequence data, its assignment to the Asiatyphlopinae remains tentative.

\section{Genus Grypotyphlops Peters, 1881}

Indian Beaked Blindsnakes

Type species. Onychocephalus acutus Duméril \& Bibron, 1844:333, by original designation.

Diagnosis. Species of Grypotyphlops have (1) eye, distinct or indistinct, (2) snout, beaked, (3) head scale arrangement, non-circular, (4) frontorostral, absent, (5) nasal, completely or incompletely divided, (6) nasal suture origin, 2nd supralabial, (7) suboculars or subpreoculars, present, (8) postoculars, 4 (rarely 3 or 5; average, 4.0), (9) preocular-labial contact, supralabials $2 \& 3,(10)$ midbody scale rows, 24-30, (11) scale row reduction, present, (12) total scale rows, 448-526 (average, 487), (13) caudals, 7-13 (average, 10.0), (14) maximum total length, $630 \mathrm{~mm}$, (15) total length/midbody diameter, 30-66 (average, 48.0), (16) total length/tail length, 17-133 (average, 75.1), (17) dorsal color, gray, brown, or golden brown, (18) ventral color, grayish-white, yellow, or pale brown, (19) dorsum darker than venter, (20) overall, coloration is uniform, although pale scale centers may appear as weakly-defined lines (Tables 1-2); no molecular phylogenetic information is available.

From other genera of Asiatyphlopinae except Acutotyphlops and Cyclotyphlops, Grypotyphlops differs in having subocular scales (versus absent). Grypotyphlops differs from Acutotyphlops in lacking a frontorostral and from Cyclotyphlops in having non-circular head scales (versus circular arrangement). Although one species of $X e-$ rotyphlops has a subocular scale, Grypotyphlops differs from that genus in having more postoculars (3-5 versus 2), more midbody scale rows (29 versus 23.5 , averages), and more total scale rows (448-526 versus 206-435). At 630 $\mathrm{mm} \mathrm{TL}$, Grypotyphlops also stands out in its large size.

Content. One species: Grypotyphlops acutus (Table 3).

Distribution. Grypotyphlops is distributed in peninsular India south of the Ganges and Rajputana river basins. 
Etymology. The generic name is a masculine noun formed from the Greek adjective grypos (hook-nosed) and Greek noun typhlops (the blind), in reference to the beaked snouts of these blindsnakes.

Remarks. We agree with Dixon \& Hendricks (1979) that the type locality of another beaked blindsnake, Onychocephalus unilineatus Duméril \& Bibron, is in error and unlikely from French Guiana. We also agree with Wallach (2003) that O. unilineatus is a synonym of Grypotyphlops acutus, and that recognition of Grypotyphlops is warranted based on morphology.

\section{Genus Indotyphlops gen. nov.}

South Asian Blindsnakes

Type species. Typhlops pammeces Günther, 1864:444.

Diagnosis. Species of Indotyphlops have (1) eye, distinct (sometimes indistinct), (2) snout, rounded, (3) head scale arrangement, non-circular, (4) frontorostral, absent, (5) nasal, completely or incompletely divided, (6) nasal suture origin, 2nd supralabial (sometimes preocular, or rarely 1st supralabial), (7) suboculars or subpreoculars, absent, (8) postoculars, 1 in all 18 species recorded, but variable (1-2) in 1 of those species, (9) preocular-labial contact, supralabials 2 \& 3 (sometimes 3rd only), (10) midbody scale rows, 18-20 (22 in 1 species; average, 19.4), (11) scale row reduction, absent (sometimes present), (12) total scale rows, 229-468 (average, 345), (13) caudals, 7-15 (average, 10.8), (14) maximum total length, 91-285 (average, 175) mm, (15) total length/midbody diameter, 28-130 (average, 57.6), (16) total length/tail length, 22.3-76 (average, 46.4), (17) dorsal color, shades of brown (sometimes cream, gray, yellowish-brown, reddish brown, lavender-gray, or black), (18) ventral color, variable (white, cream, pale brown, lavender-gray), (19) dorsum darker than venter, (20) overall, uniform, but often with a darker median row of scales giving a slight lineate appearance (Tables 1-2); molecular phylogenetic support (Fig. 1).

From other genera of Asiatyphlopinae, Indotyphlops differs in having a single postocular (versus 2 or more). Exceptions are Cyclotyphlops, 3 species of Anilios, and 3 species of Ramphotyphlops. It also has the lowest number of midbody scales (19.4 versus 20.1-30.4 in others; averages), and no scale reduction (6 of 7 species recorded; versus reduction present in Acutotyphlops, Cyclotyphlops, Malayotyphlops, Grypotyphlops, and Xerotyphlops). In total length, Indotyphlops is one of the smallest genera ( $\mathrm{TL}=175 \mathrm{~mm}$ versus $>243 \mathrm{~mm}$ in all others except Cyclotyphlops). It is also the thinnest genus in the subfamily (TL/MBD $=57.6$ versus $<56$ in other genera). Indotyphlops lacks yellow on the venter (except one species) whereas Asiatyphlops, with which it is broadly sympatric, has yellow on the venter.

Content. Twenty-two species: Indotyphlops ahsanai, I. albiceps, I. braminus, I. exiguus, I. filiformis, I. fletcheri, I. hypsobothrius, I. jerdoni, I. khoratensis, I. Iankaensis, I. lazelli, I. loveridgei, I. madgemintonae, I. malcolmi, I. meszoelyi, I. ozakiae, I. pammeces, I. porrectus, I. schmutzi, I. tenebrarum, I. veddae, and I. violaceus (Table 3).

Distribution. Indotyphlops is broadly distributed in southern and southeastern Asia, including Bhutan, Cambodia, southern China, India and Sri Lanka, Indonesia, Laos, Malaysia, Myanmar, Nepal, and Thailand. Most species occur in South Asia, especially India and Sri Lanka. One invasive species, I. braminus, now has a nearly global distribution.

Etymology. The generic name is a masculine noun formed from the adjective indianus ( $a$, um; i.e., 'from India') and the Greek noun typhlops (the blind).

Remarks. Blindsnakes from southern and southeastern Asia have proven especially difficult to classify in the past using morphology, such that there has been little agreement (McDowell 1974; Khan 1999; Wallach 1999; 2000; Wallach \& Pauwels 2004). Species have generally been placed in two genera, Ramphotyphlops and Typhlops. However, with molecular phylogenetic evidence, Vidal et al. (2010) showed that the "Typhlops ater Group" represents a well-defined and deeply-divergent lineage that they named as a separate family, Gerrhopilidae. That study and subsequent molecular studies (Marin et al. 2013a; Marin et al. 2013b) (Figs. 1, 2) have allowed us to further clarify the taxonomy, while recognizing that molecular sampling is still sparse. In addition to allocating species to Anilios, Malayotyphlops, Ramphotyphlops s.s., and Sundatyphlops, we have erected Asiatyphlops and 
Indotyphlops for the remaining species, most of which occur on the mainland of southern and southeast Asia. The relationships of species within Indotyphlops are not yet established, but the close relationship of the globally invasive species I. braminus to an Indian species (I. pammeces) suggests an origin in India. See also Remarks for Asiatyphlops concerning allocation of $A$. leucomelas.

\section{Genus Malayotyphlops gen. nov.}

Malay Archipelago Blindsnakes

Type species. Typhlops luzonensis Taylor, 1919:105.

Diagnosis. Species of Malayotyphlops have (1) eye, distinct (rarely indistinct), (2) snout, rounded, (3) head scale arrangement, non-circular, (4) frontorostral, absent, (5) nasal, incompletely divided (sometimes completely divided), (6) nasal suture origin, 2nd supralabial (rarely, 1st supralabial), (7) suboculars or subpreoculars, absent (rarely present), (8) postoculars, 2-4 (average, 2.75), (9) preocular-labial contact, supralabials $2 \& 3$ or 3rd only (rarely 2nd only), (10) midbody scale rows, 24-30 (average, 26.8), (11) scale row reduction, present, (12) total scale rows, 300-461 (average, 367), (13) caudals, 9-14 (average, 11.9), (14) maximum total length, 122-445 (average, 256) $\mathrm{mm},(15)$ total length/midbody diameter, 31-58 (average, 42.0), (16) total length/tail length, 28-71 (average, 51.8), (17) dorsal color, variable (brown, gray-brown, reddish brown, greenish-black, black), (18) ventral color, variable (cream, yellowish, red, pink, pinkish-yellow), (19) dorsum darker than venter, (20) overall, patternless or with longitudinal lines, series of spots, or collar (Tables 1-2); only one species is included in the molecular phylogeny (Fig. 1).

In lacking a protrusible hemipenis and retrocloacal sacs (McDowell 1974), Malayotyphlops is separated from most other blindsnakes occurring in Southeast Asia, Indonesia, Australasia, and the western Pacific: Acutotyphlops, Anilios, Cyclotyphlops, Ramphotyphlops, and Sundatyphlops. From other genera of Asiatyphlopinae, Malayotyphlops differs from Acutotyphlops in lacking a frontorostral and from Cyclotyphlops in having non-circular head scales (versus circular arrangement). It differs from Grypotyphlops in lacking subocular scales. It differs from Cyclotyphlops and Indotyphlops in having more postoculars (2.75 versus 1.0-1.03; averages). It differs from Anilios, Cyclotyphlops, Indotyphlops, Ramphotyphlops, Sundatyphlops, and Xerotyphlops in having higher average midbody scale rows (26.8 versus 19.4-23.5). It differs from Anilios, Grypotyphlops, and Sundatyphlops in having fewer total scale rows (367 versus 466-496, averages) and smaller body size ( $T L=256$ versus 353-630; averages). See also comments below regarding other potential diagnostic characters.

Content. Ten species: Malayotyphlops canlaonensis, M. castanotus, M. collaris, M. hypogius, M. koekkoeki, M. kraali, M. luzonensis, M. manilae, M. ruber, and M. ruficauda (Table 3).

Distribution. Malayotyphlops is distributed widely in the Malay Archipelago, including the Philippines (7 species), Bunyu Island off of northern Borneo (1 species), and Seram and Kai in the Maluku Islands (1 species).

Etymology. The generic name is a masculine compound noun formed from the adjective malayanus ( $a$, um; i.e.,' 'from the Malay Archipelago') and the Greek noun typhlops (the blind).

Remarks. This genus corresponds to the "Typhlops ruficauda Group" of McDowell (1974), who pointed out the reduction in the second supralabial in these species as a potential diagnostic character. This is also reflected in our scoring of preocular-labial contact with supralabial 3 (only) in 5 of the 8 species, instead of the usual contact with supralabials 2-3. Other potential diagnostic characters include the absence of a rectal caecum and fusion of glandular lines underlying the postnasal and preocular (McDowell 1974; Wynn \& Leviton 1993), although more taxa will need to be surveyed to assess the usefulness of these characters. In the past, several authors incorrectly amended, as ruficaudus, the species name (a noun) of Malayotyphlops ruficauda to match the gender of the genus. Although Taylor (1919) placed "Typhlops manilae" in the "ater Group," now Gerrhopilidae (Vidal et al. 2010), we place it in Malayotyphlops. Taylor's only reason for that placement was presence of a subocular scale. Subocular scales are common (not constant) in Gerrhopilidae but they are also common in several other genera and rare in yet other genera. Otherwise, the characters of M. manilae are more consistent with traits of Malayotyphlops, especially 
in high midbody scale rows (28, versus 18 in Gerrhopilidae) and short tail (TL/TA $=56$ versus $~ 20-30$ ).

\section{Genus Ramphotyphlops Fitzinger, 1843}

Western Pacific Blindsnakes

Type species. Typhlops multilineatus Schlegel, 1839:40, by original designation.

Diagnosis. Species of Ramphotyphlops have (1) eye, distinct (rarely indistinct), (2) snout, rounded or beaked, (3) head scale arrangement, non-circular, (4) frontorostral, absent, (5) nasal, completely or incompletely divided, (6) nasal suture origin, supralabial 2 (sometimes 1 or $1 / 2$ suture), (7) suboculars or subpreoculars, absent (rarely present), (8) postoculars, 2-3 (rarely 1; average, 2.15), (9) preocular-labial contact, supralabials 2-3 (rarely 3 only or fused with ocular or nasal), (10) midbody scale rows, 18-30 (average, 21.7), (11) scale row reduction, present or absent, (12) total scale rows, 206-653 (average, 419), (13) caudals, 8-36 (average, 19.8), (14) maximum total length, 117-480 (average, 302) mm, (15) total length/midbody diameter, 18-91.5 (average, 48.8), (16) total length/ tail length, 16.7-70 (average, 31.8), (17) dorsal color, usually dark brown (sometimes medium brown, tan, gray, or purplish), (18) ventral color, usually cream or yellowish-white (sometimes gray-green, pinkish, tan, or brown), (19) dorsum darker than venter, (20) overall, usually either patternless or with longitudinal lines (Tables 1-2); molecular phylogenetic support (Fig. 1).

Ramphotyphlops is part of a larger, more inclusive clade (Ramphotyphlops s.l.) that shares a solid, awnlike protrusible hemipenis and retrocloacal sacs (Robb 1966; McDowell 1974), although not all species have been examined for those traits. Presumably these characters distinguish it from other genera in the Asiatyphlopinae, except those three genera formerly in Ramphotyphlops s.l. and thus the closest relatives of Ramphotyphlops s.s.: Acutotyphlops, Anilios, and Sundatyphlops. From Acutotyphlops, Ramphotyphlops can be distinguished by lacking a frontorostral scale. From Anilios, it can be distinguished by its smaller maximum size (mean among species, 302 versus $354 \mathrm{~mm} \mathrm{TL}$ ), higher mean number of midbody scales (21.7 versus 20.1), and longer tails (TL/TA $=31.8$ versus 49.7; averages). From Sundatyphlops, it can be distinguished by its smaller maximum size (302 versus $395 \mathrm{~mm} \mathrm{TL;}$ only $R$. angusticeps, $455 \mathrm{~mm} \mathrm{TL}$, has a greater maximum size).

Content. Twenty-one species: Ramphotyphlops acuticauda, R. adocetus, R. angusticeps, R. becki, R. conradi, $R$. cumingii, $R$. depressus, $R$. exocoeti, $R$. flaviventer, $R$. hatmaliyeb, $R$. lineatus, $R$. lorenzi, $R$. melanocephalus, $R$. mansuetus, R. marxi, R. multilineatus, R. olivaceus, R. similis, R. suluensis, R. supranasalis, and R. willeyi (Table 3).

Distribution. Ramphotyphlops is distributed broadly on many islands in the western Pacific Ocean, north and south of the Equator (especially in Indonesia, New Guinea, Philippines, and the Solomon Islands, but on many other islands as well) and with one species occurring in Southeast Asia.

Etymology. The generic name is a masculine noun formed from the Greek adjective ramphos (curved beak or bill) and Greek noun typhlops (the blind), in reference to the pointed or beak-like snout in most species of this genus.

Remarks. We include Ramphotyphlops conradi (Sulawesi), usually assigned to Typhlops, because it shares more characters with this genus than with others, and because of its distribution. We also place Cathetorhinus melanocephalus in this genus, contra Wallach \& Pauwels (2008). Those authors argued that it was excluded from Ramphotyphlops because it has a different supralabial imbrication pattern, broad rostral, lack of superior nasal suture, and black head. Those scalation characters are unusual, although they are likely inter-related in an evolutionary sense, and thus not trenchant. Otherwise most of the characters of this species are consistent with the genus Ramphotyphlops, including beaked head, low midbody scales, no scale reduction, high total scale rows, high caudals, and long tail.

\section{Genus Sundatyphlops gen. nov.}

Lesser Sunda Blindsnakes 
Type species. Typhlops polygrammicus Schlegel, 1839:40.

Diagnosis. Species of Sundatyphlops have (1) eye, distinct, (2) snout, rounded, (3) head scale arrangement, non-circular, (4) frontorostral, absent, (5) nasal, incompletely or completely divided, (6) nasal suture origin, supralabial 1,2 , or $1 / 2$ suture, (7) suboculars or subpreoculars, unreported, (8) postoculars, unreported, (9) preocular-labial contact, supralabials 2-3, (10) midbody scale rows, 22, (11) scale row reduction, unreported, (12) total scale rows, 496, (13) caudals, 16, (14) maximum total length, $395 \mathrm{~mm}$, (15) total length/midbody diameter, 33-58 (average, 45.5), (16) total length/tail length, 15-52 (average, 33.3), (17) dorsal color, usually dark brown, olive, olive gray, or pale yellow, (18) ventral color, whitish, whitish-pink, or pale yellow, (19) dorsum darker than (rarely similar to) venter, (20) pattern of longitudinal dark lines on yellow ground color, sometimes with same lineate pattern (but paler) on venter (Tables 1-2); only one species is included in the molecular phylogeny (Fig. 1).

As noted in the previous account, Sundatyphlops shares with Acutotyphlops, Anilios, and Ramphotyphlops a solid, awn-like protrusible hemipenis and retrocloacal sacs (Robb 1966; McDowell 1974), although not all species have been examined for those traits. From Acutotyphlops, Sundatyphlops can be distinguished by lacking a frontorostral scale. From Anilios, it can be distinguished by its larger maximum size (mean among species, 395 versus $353 \mathrm{~mm} \mathrm{TL}$ ), higher mean number of midbody scales (22 versus 20.1), and longer tails (TL/TA = 33.3 versus 49.7; averages). From Ramphotyphlops, it can be distinguished by its higher total scale rows (496 versus 419; averages) and larger maximum size (395 versus $302 \mathrm{~mm}$ TL; only R. angusticeps, $455 \mathrm{~mm} \mathrm{TL}$, has a greater maximum size).

Content. A single species, Sundatyphlops polygrammicus (Table 3).

Distribution. Sundatyphlops is distributed in the Lesser Sunda Islands, including Flores, Komodo, Lombok, Moyo (near Sumbawa), Sumba, Sumbawa, and Timor-Leste, and West Timor (De Lang 2011).

Etymology. The generic name is a masculine noun formed from the adjective sundanus ( $a$, um; i.e., 'from Sunda') and the Greek noun typhlops (the blind).

Remarks. See Remarks for Anilios regarding our distinction of the Lesser Sunda populations of Anilios polygrammicus s.l. as a species distinct from populations inhabiting northeastern Australia (Queensland) and southern New Guinea which we assign to Anilios torresianus (comb. nov.). Not only are they different species, but we place them in different genera. Remarkably, the species A. torresianus is more closely related to all other Australian species (potentially $90+$ species; Fig. 2) than it is to populations once considered conspecific, here called Sundatyphlops polygrammicus. Two potentially diagnostic characters identified by us are total middorsal scales (496 in S. polygrammicus versus 365 in A. torresianus) and the pattern of 11 black lines on a yellow ground color that gave S. polygrammicus its name (versus a braided pattern of pale-edged scales in A. torresianus). However, both species are in need of more systematic work, as it is quite likely that each is complex of species, given their distributions on multiple land masses. Several synonyms exist for S. polygrammicus (type locality, "Timor"), which we consider as subspecies following De Lang (2011): S. p. elberti (Roux 1911) from Lombok, S. p. florensis (Boulenger 1897) from Flores, S. p. brongersmai (Mertens 1929) from Sumba, S. p. undecimlineatus (Mertens 1927) from Sumbawa; $S$. soensis (De Jong 1930) from Timor apparently is a synonym of S. p. polygrammicus. See also accounts by Mertens (1929) and Focart (1953), and allocation of subspecies to islands by De Lang (2011). As McDowell (1974) pointed out, most authors who have reviewed specimens of $A$. polygrammicus s.l. in the past have confused characters of different populations (here, different genera). Hence a careful review of the museum material is needed. Here, we use only character data noted from specimens of known locality. The lineate pattern was described for snakes from Flores (S. p. florensis), Sumbawa (S. p. undecimlineatus), and Lombok (S. p. elberti), with apparently only faint lines in Sumba snakes (S. p. brongersmai). The brief description of the type material by Schlegel (1839), from Timor, does not mention lines specifically, although his name (polygrammicus) infers that it was also lineate. However, he mentioned that the dark brown scales have a "yellow edge," and by that it must be inferred that the lines are one scale wide and formed by parallel yellow edges that do not include the scale tip. This is an important distinction, because populations in Australia previously confused with S. polygrammicus have a braided pattern (Cogger 2000), whereby the entire scale edge is pale, instead of just the parallel edges. Considering all of this, and that the lineate 
pattern has been associated with snakes from widely scattered localities in the Lesser Sunda Islands, we assume, tentatively, that it is diagnostic of this species and genus, even if it was not explicitly mentioned in all accounts. Our DNA sample comes from Moyo, an islet off of Sumbawa, and presumably S. p. undecimlineatus (De Lang 2011).

\section{Genus Xerotyphlops gen. nov.}

Desert Blindsnakes

Type species. Typhlops vermicularis Merrem, 1820:158.

Diagnosis. Species of Xerotyphlops have (1) eye, distinct, (2) snout, rounded, (3) head scale arrangement, non-circular, (4) frontorostral, absent, (5) nasal, incompletely divided, (6) nasal suture origin, supralabial 2, (7) suboculars or subpreoculars, absent (rarely present), (8) postoculars, 2 (average, 2.0), (9) preocular-labial contact, supralabials $2 \& 3,(10)$ midbody scale rows, $20-24$ (average, 23.5), (11) scale row reduction, present, (12) total scale rows, 206-435 (average, 355), (13) caudals, 10-16 (average, 13.0), (14) maximum total length, 220-350 (average, 292) $\mathrm{mm},(15)$ total length/midbody diameter, 37-55 (average, 45.6), (16) total length/tail length, 88, (17) dorsal color, whitish or brown, (18) ventral color, whitish, cream, or brown, (19) dorsum darker than (or same as) venter, (20) overall, either patternless or darker longitudinal lines, (Tables 1-2); molecular phylogenetic support (Fig. 1).

From other genera of Asiatyphlopinae, Xerotyphlops has the shortest tail (TL/TA = 87.7 versus 31.1-75.1; averages). It differs from Acutotyphlops in lacking a frontorostral and from Cyclotyphlops in having non-circular head scales (versus circular arrangement). It differs from Grypotyphlops in lacking subocular scales (rarely present). It differs from Cyclotyphlops and Indotyphlops in having more postoculars (2.00 versus 1.0-1.03; averages). It differs from Anilios, Indotyphlops, and Ramphotyphlops in having higher average midbody scale rows (23.5 versus 19.421.7; averages). It differs from Anilios, Grypotyphlops, and Sundatyphlops in having fewer total scale rows (355 versus 466-496; averages) and smaller body size (TL = 292 versus 353-630; averages). It differs from Malayotyphlops in having lower average midbody scales (23.5 versus 26.8; averages). It differs from Asiatyphlops and Cyclotyphlops in having a thinner body (TL/MBD $=45.6$ versus $35.1-35.2$; averages).

Content. Four species: Xerotyphlops etheridgei, $X$. socotranus, $X$. vermicularis, and $X$. wilsoni (Table 3 ).

Distribution. Xerotyphlops is distributed in xeric regions of three continents, including the Sahara Desert and Socotra Island (Africa), southwestern Asia, and southeastern Europe. Although the genus occurs in xeric regions, at least one species (X. vermicularis) apparently prefers more humid microhabitats (Kornilios et al. 2011), which is not uncommon for organisms occurring in desert regions. Another species, $X$. socotranus, often inhabits areas with little moisture and vegetation (Rösler \& Wranik 2004; Razzetti et al. 2011).

Etymology. The generic name is a masculine noun formed from the Greek adjective xeros (desert) and Greek noun typhlops (the blind), in reference to the desert region where these species occur. As an alternate meaning, the rarity of species in this large region could be described as a 'blindsnake desert.'

Remarks. The molecular phylogeny of Vidal et al. (2010), and the updated phylogeny here (Fig. 1), show that Xerotyphlops is an early offshoot of typhlopid snakes. We include Xerotyphlops etheridgei from Mauritania, which is a geographic outlier (Wallach 2002), and X. wilsoni, known only from the lost holotype (McDiarmid et al. 1999). A photograph in life of $X$. socotranus shows the lineate pattern (Razzetti et al. 2011). There are likely additional species confused within X. vermicularis (Kornilios et al. 2011; Kornilios et al. 2013).

\section{Subfamily Madatyphlopinae subfam. nov.}

Malagasy Blindsnakes

Type genus. Madatyphlops gen. nov.

Diagnosis. As for the genus.

Content. One genus: Madatyphlops gen. nov. (Table 3).

Distribution. Madatyphlopinae is only known from Madagascar. 
Etymology. As for the genus.

\section{Genus Madatyphlops gen. nov.}

Malagasy Blindsnakes

Type species. Onychocephalus arenarius Grandidier, 1872:9.

Diagnosis. Species of Madatyphlops have (1) eye, distinct, (2) snout, rounded, (3) head scale arrangement, non-circular, (4) frontorostral, absent, (5) nasal, incompletely (sometimes completely) divided, (6) nasal suture origin, supralabial 2, (7) suboculars or subpreoculars, absent, (8) postoculars, 2-3 (rarely 1 or 4; average, 2.27), (9) preocular-labial contact, supralabials $2 \& 3$, (10) midbody scale rows, 20-28 (average, 22.9), (11) scale row reduction, present or absent, (12) total scale rows, 252-600 (average, 429), (13) caudals, 6-18 (average, 11.2), (14) maximum total length, 176-600 (average, 320) mm, (15) total length/midbody diameter, 27-85 (average, 45.6), (16) total length/tail length, 33-125 (average, 53.7), (17) dorsal color, usually brown but variable (sometimes white, pink, cream, yellow, gray), (18) ventral color, variable (white, pink, cream, yellow, gray, brown), (19) dorsum darker than venter, (20) overall, usually patternless (rarely with line or yellow nuchal collar) (Tables 1-2); molecular phylogenetic support (Fig. 1).

Madatyphlops is distinguished from all genera of Afrotyphlopinae in having fewer postocular scales (2.27 versus 3.68), fewer midbody scales (22.9 versus 24.8), smaller body size (320 versus $403 \mathrm{~mm} \mathrm{TL}$ ), and longer tails (TL/TA = 53.7 versus 68.2; averages). It is distinguished from all genera of Typhlopinae in having more postocular scales (2.27 versus 1.66), more midbody scales (22.9 versus 20.3 ), more total scale rows ( 429 versus 369; averages), and larger body size (320 versus $264 \mathrm{~mm}$ TL). Madatyphlops is distinguished from individual genera of Asiatyphlopinae in the following ways: Acutotyphlops has a frontorostral and complete nasal division (versus absent, and suture incomplete), Cyclotyphlops has a circular arrangement of head scales (versus non-circular), Grypotyphlops has suboculars present (versus absent), Indotyphlops has 1 postocular (versus 2-3), Asiatyphlops, Malayotyphlops, and Xerotyphlops have fewer average total scale rows (339, 367, and 355 versus 429), Ramphotyphlops and Sundatyphlops have longer tails (TL/TA = 31.8 and 33.3 versus 53.7; averages), and Anilios has a solid, awn-like protrusible hemipenis and retrocloacal sacs (versus non-protrusible hemipenis and retrocloacal sacs absent). Xenotyphlopidae (Xenotyphlops) also occurs on Madagascar, but it differs from Madatyphlops in having a circular rostral scale and single enlarged anal shield (Vidal et al. 2010).

Content. Eleven species: Madatyphlops andasibensis, M. arenarius, M. boettgeri, M. decorsei, M. domerguei, M. madagascariensis, M. microcephalus, M. mucronatus, M. ocularis, M. rajeryi, and M. reuteri (Table 3).

Distribution. Madatyphlops is known only from Madagascar.

Etymology. The generic name is a masculine noun formed from the adjective madagascarianus ( $a$, um; 'from Madagascar') and the Greek noun typhlops (the blind).

Remarks. Madatyphlops is represented in the molecular phylogeny of Vidal et al. (2010), and in the tree here (Fig. 1), by two described species: $M$. andasibensis and $M$. arenarius. These two species are consistent with other typhlopid species in Madagascar, previously assigned to the genus Typhlops (McDiarmid et al. 1999; Glaw \& Vences 2007; Vidal et al. 2010), in having a distinct eye, rounded snout, lacking suboculars, having the nasal suture origin at the 2nd supralabial, and in having the nasal incompletely divided (completely divided in M. ocularis). Although variable in color, they tend to lack a pattern (Glaw \& Vences 2007).

\section{Subfamily Typhlopinae Merrem, 1820}

New World Blindsnakes

Type genus. Typhlops Oppel, 1811.

Diagnosis. Members of this subfamily have (1) eye, distinct (sometimes indistinct), (2) snout, rounded (rarely acuminate), (3) head scale arrangement, non-circular, (4) frontorostral, absent, (5) nasal, completely divided 
(sometimes incompletely divided), (6) nasal suture origin, supralabial 2 (rarely 1 or $1 / 2$ suture), (7) suboculars or subpreoculars, absent (rarely present), (8) postoculars 1-4 (average, 1.66), (9) preocular-labial contact, supralabials $2 \& 3$ or 3rd only (rarely none), (10) midbody scale rows, 16-24 (average, 20.3), (11) scale row reduction, present or absent, (12) total scale rows, 170-629 (average, 369), (13) caudals, 6-22 (average, 11.7), (14) maximum total length, 101-522 (average, 264) mm, (15) total length/midbody diameter, 16-77 (average, 42.7), (16) total length/ tail length, 18-270 (average, 48.7), (17) dorsal color, brown (sometimes cream, tan, yellowish, or unpigmented), (18) ventral color, unpigmented (sometimes white, cream, or rarely brown), (19) dorsum darker than venter, (20) overall, usually patternless snakes, but rarely with indistinct spots, lines, and reticulations (Tables 1-2); molecular phylogenetic support (Figs. 1, 3).

The subfamily Typhlopinae is distinguished from Afrotyphlopinae in having fewer postocular scales (1.66 versus 3.69; averages), fewer midbody scales (20.3 versus 24.8), fewer total scale rows (369 versus 437), smaller body size (264 versus $403 \mathrm{~mm} \mathrm{TL}$ ), stouter body (TL/MBD 42.7 versus 52.4), and longer tails (TL/TA 48.7 versus 68.2). It is distinguished from Asiatyphlopinae in having fewer postocular scales (1.66 versus 2.19 ), fewer midbody scales (20.3 versus 21.8 ), and fewer total scale rows ( 369 versus 408 ). It is distinguished from the Madatyphlopinae in having fewer postocular scales (1.66 versus 2.27 ), fewer midbody scales (20.3 versus 22.9 ), fewer total scale rows (369 versus 429), and smaller body size (264 versus $320 \mathrm{~mm} \mathrm{TL}$ ).

Content. Four genera: Amerotyphlops gen. nov., Antillotyphlops gen. nov. , Cubatyphlops gen. nov., and Typhlops Oppel 811 (Table 3).

Distribution. The subfamily Typhlopinae is distributed in the New World tropics, from eastern Mexico (Veracruz) to southern South America (Bolivia and Argentina), with a large radiation on Caribbean islands.

Etymology. The subfamily name derives from the genus Typhlops, which is a Greek noun (the blind).

Remarks. This is the best known subfamily of blindsnakes, because it is the most densely sampled group with molecular data. However, no molecular data have been collected for any species of the genus Amerotyphlops in Middle America.

\section{Genus Amerotyphlops gen. nov.}

American Blindsnakes

Type species. Typhlops brongersmianus Vanzolini, 1976:247.

Diagnosis. Species of Amerotyphlops have (1) eye, distinct or indistinct, (2) snout, rounded, (3) head scale arrangement, non-circular, (4) frontorostral, absent, (5) nasal, completely or incompletely divided, (6) nasal suture origin, supralabial 2 (sometimes 1 or $1 / 2$ suture), (7) suboculars or subpreoculars, absent (rarely present), (8) postoculars, 1-2 (rarely 3-4, average, 1.69), (9) preocular-labial contact, supralabials $2 \& 3$, (10) midbody scale rows, 16-22 (average, 19.1), (11) scale row reduction, absent (rarely present), (12) total scale rows, 170-556 (average, 327), (13) caudals, 6-15 (average, 9.4), (14) maximum total length, 101-522 (average, 287) mm, (15) total length/ midbody diameter, 16-77 (average, 38.7), (16) total length/tail length, 23-270 (average, 66.8), (17) dorsal color, brown or yellow, (18) ventral color, white, cream, or yellow, (19) dorsum darker than venter, (20) overall color pattern often consists of spots, lines, reticulations, and/or a band over region of eyes (Tables 1-2); molecular phylogenetic support (Figs. 1, 3).

This genus is distinguished from two other genera of the subfamily Typhlopinae, Antillotyphlops and Typhlops, in that the preocular contacts supralabials 2 and 3 (versus preocular contact with supralabial 3 only) (Thomas 1968; 1976; Dixon \& Hendricks 1979; Thomas \& Hedges 2007). Although Amerotyphlops can be distinguished from those genera, it is more difficult to separate it from the primarily Cuban genus Cubatyphlops. It shares with that genus the preocular contact with supralabials 2 and 3, and presence of a single postocular in some species. However, it can be nearly completely distinguished from Cubatyphlops in total scale rows: there are minimally 453 in 11 of the 12 species of that genus (C. caymanensis, 351-408) whereas in Amerotyphlops, the maximum number of total scale rows is 441 , except in one species, A. microstomus (487-556). Concerning the two overlap species, $C$. 
caymanensis is separated from Amerotyphlops in the molecular phylogeny, and A. microstomus is separated from Cubatyphlops in having 2 postoculars instead of 1 postocular. Also, 8 of the 14 species of Amerotyphlops can be distinguished from Cubatyphlops in having either an incompletely divided nasal scale (A. minuisquamus, A. paucisquamus, A. reticulatus, and A. yonenagae) or a patterned (lines or spots) dorsum and/or head (A. brongersmianus, A. minuisquamus, A. paucisquamus, A. reticulatus, A. tasymicris, A. tenuis, A. trinitatus, and A. yonenagae) or both. In Cubatyphlops, the nasal is completely divided and there is no distinct pattern.

Content. Fourteen species: Amerotyphlops amoipira, A. brongersmianus, A. costaricensis, A. lehneri, A. microstomus, A. minuisquamus, A. paucisquamus, A. reticulatus, A. stadelmani, A. tasymicris, A. tenuis, A. trinitatus, A. tycherus, and $A$. yonenagae (Table 3 ).

Distribution. Amerotyphlops is distributed in the New World, primarily on the mainland, ranging from eastern Mexico (Veracruz) to southern South America (Bolivia and Argentina), and includes a West Indian species, A. tasymicris in Grenada and the Grenadines.

Etymology. The generic name is a masculine noun formed from the adjective americanus ( $a$, um; 'from America') and Greek noun typhlops (the blind).

Remarks. Molecular data are available for only 3 of 14 species in this genus, which remains a gap in knowledge. Nonetheless, the suite of characters shared by the species, noted above, indicates that it is a monophyletic group with a geographic cohesiveness.

\section{Genus Antillotyphlops gen. nov.}

Antillean Blindsnakes

Type species. Typhlops hypomethes Hedges \& Thomas, 1991:452.

Diagnosis. Species of Antillotyphlops have (1) eye, distinct, (2) snout, rounded (rarely acuminate), (3) head scale arrangement, non-circular, (4) frontorostral, absent, (5) nasal, completely divided, (6) nasal suture origin, supralabial 2, (7) suboculars or subpreoculars, absent, (8) postoculars, 2 (rarely 1; average 2.0), (9) preocular-labial contact, supralabial 3, (10) midbody scale rows, 16-24 (average, 20.5), (11) scale row reduction, present, (12) total scale rows, 299-499 (average, 378), (13) caudals, 11-14 (average, 12.3), (14) maximum total length, 110-360 (average, 233) mm, (15) total length/midbody diameter, 37-70 (average, 46.8), (16) total length/tail length, 30-61 (average, 44.5), (17) dorsal color, brown, grayish-brown, or yellow, (18) ventral color, white or cream (rarely brown), (19) dorsum darker than venter, and (20) overall, lacking any distinctive pattern (spots, lines, or stripes), although rarely faint trace of lines (Tables 1-2); molecular phylogenetic support (Figs. 1, 3).

Antillotyphlops is distinguished from Cubatyphlops by the presence of 2 postoculars (versus 1 ) and preocular contact with supralabial 3 only (versus contact with supralabials 2 and 3 in Cubatyphlops). The same distinction holds for Antillotyphlops versus the more distantly related Amerotyphlops, although 4 species of that genus have more than 1 postocular (Thomas 1968; 1976; Dixon \& Hendricks 1979; Thomas \& Hedges 2007). A closer comparison is needed between Antillotyphlops and Typhlops. With a cladistic analysis of morphological characters, Thomas (1989) found that species placed here in the genus Antillotyphlops (A. dominicana, A. granti, A. monensis, and $A$. richardi) formed a group based on the sharing of attenuate hemipenes, although he included Typhlops sulcatus in that group and placed A. monastus in a separate group with T. jamaicensis (hemipene data are not available for some species). Also, Antillotyphlops have more total scale rows than Typhlops (378 versus 312; averages) and are thinner-bodied (TL/MBD $=46.8$ versus 35.9; averages).

Content. Twelve species: Antillotyphlops annae, A. catapontus, A. dominicanus, A. geotomus, A. granti, A. guadeloupensis, A. hypomethes, A. monastus, A. monensis, A. naugus, A. platycephalus, and A. richardi (Table 3).

Distribution. Antillotyphlops is distributed on islands in the eastern Caribbean, including Puerto Rico (and its satellite islets), the U.S. and British Virgin Islands, the Turks and Caicos Islands, and the Lesser Antilles (south to Dominica).

Etymology. The generic name is a masculine noun formed from the adjective antilleus ( $a$, um; 'from the 


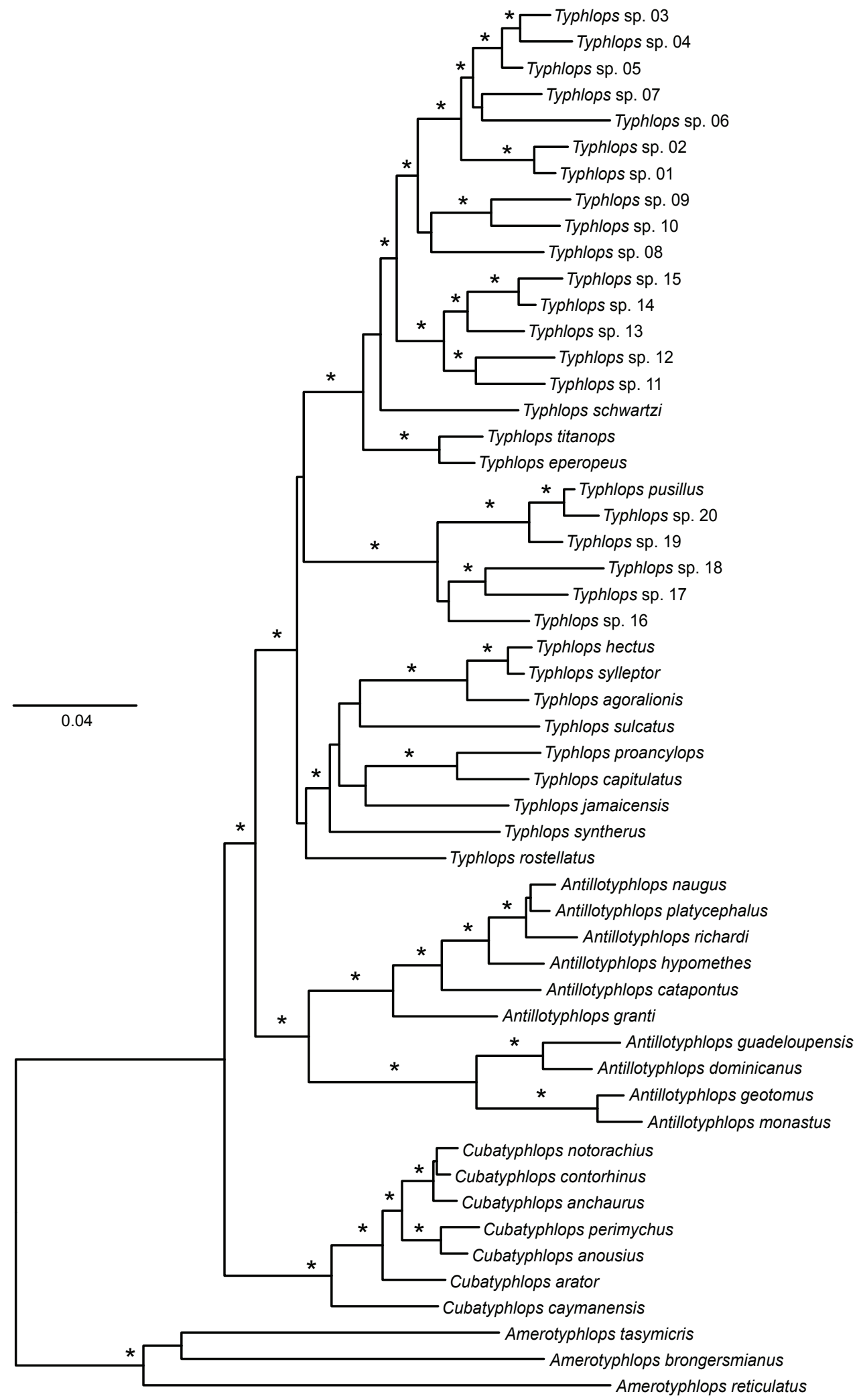

Figure 3. Phylogenetic ML tree of typhlopid snakes from Caribbean islands based on an analysis of DNA sequences of 6 nuclear protein-coding genes and 4 mitochondrial genes (dataset C). Nodes with asterisks are supported by posterior probability $>95 \%$ and ML bootstrap probability $>70 \%$. The tree is rooted with Indotyphlops braminus (not shown). 
Antilles') and Greek noun typhlops (the blind).

Remarks. Molecular data are available for nearly all known species in this genus and its monophyly is well supported. It is also a geographically cohesive group.

\section{Genus Cubatyphlops gen. nov.}

Cuban blindsnakes

Type species. Typhlops biminiensis Richmond, 1955:2.

Diagnosis. Species of Cubatyphlops have (1) eye, distinct, (2) snout, rounded, (3) head scale arrangement, non-circular, (4) frontorostral, absent, (5) nasal, completely divided (rarely incomplete), (6) nasal suture origin, supralabial 2, (7) suboculars or subpreoculars, absent, (8) postoculars, 1 (rarely 2; average 1.0), (9) preocular-labial contact, supralabials $2 \& 3$ (rarely none), (10) midbody scale rows, 20-24 (average, 22.1), (11) scale row reduction, present or absent, (12) total scale rows, 351-629 (average, 503), (13) caudals, 22, (14) maximum total length, 197460 (average, 304) mm, (15) total length/midbody diameter, 36-75 (average, 54.7), (16) total length/tail length, 29-85 (average, 50.4), (17) dorsal color, brown (sometimes unpigmented, pinkish), (18) ventral color, unpigmented (pinkish) or rarely cream, (19) dorsum darker than venter, (20) overall, lacking any distinctive pattern (spots, lines, or stripes) (Tables 1-2); molecular phylogenetic support (Figs. 1, 3).

This genus is distinguished from the other two major genera inhabiting the Caribbean islands, Antillotyphlops and Typhlops, in that the preocular contacts supralabials 2 and 3, and one postocular is present (versus preocular contact with supralabial 3 only, and the presence of two postoculars) (Thomas 1968; 1976; Dixon \& Hendricks 1979; Thomas \& Hedges 2007). Species in this genus also stand out among closely related genera in being unusually thin, with individuals of all species except one having TL/MBD ratios above 50, being unusually long (up to $460 \mathrm{~mm} \mathrm{TL}$ ), and in having an unusually high number of total scale rows (to 629). Although Cubatyphlops can be distinguished from its closely related genera, it is more difficult to separate it from the primarily mainland New World genus Amerotyphlops. It shares with that genus the preocular contact with supralabials 2 and 3 , and, in some species of Amerotyphlops, the presence of a single postocular. However, it can be nearly completely distinguished from the mainland genus in having a high number of total scale rows, minimally 453 in 11 of the 12 species ( $C$. caymanensis, 351-408); in Amerotyphlops, the maximum number of total scale rows is 441, except in one species, A. microstomus (487-556). Concerning the two overlap species, C. caymanensis is separated from Amerotyphlops in the molecular phylogeny, and A. microstomus is separated from Cubatyphlops in having 2 postoculars instead of one postocular. Also, 8 of the 14 species of Amerotyphlops can be distinguished from Cubatyphlops in having either an incompletely divided nasal scale (A. minuisquamus, A. paucisquamus, and A. reticulatus, and A. yonenagae) or a patterned (lines or spots) dorsum and/or head (A. brongersmianus, A. minuisquamus, A. paucisquamus, A. reticulatus, A. tasymicris, A. tenuis, A. trinitatus, and A. yonenagae) or both. In Cubatyphlops, the nasal is completely divided and they have no distinct pattern.

Content. Twelve species: Cubatyphlops anchaurus, C. anousius, C. arator, C. biminiensis, C. caymanensis, C. contorhinus, C. epactius, C. golyathi, C. notorachius, C. paradoxus, C. perimychus, and C. satelles (Table 3).

Distribution. Cubatyphlops is distributed primarily in Cuba, but with species in the Cayman Islands and the Bahamas.

Etymology. The generic name is a masculine noun formed from the adjective cubanus ( $a$, um;'from Cuba') and Greek noun typhlops (the blind).

Remarks. Given the high total scale rows and lack of any distinctive color pattern in this genus, compared with the mainland genus Amerotyphlops, it is likely that both genera are monophyletic. 


\section{Genus Typhlops Oppel (1811)}

Antillean Blindsnakes

Type species. [Anguis] lumbricalis Linnaeus, 1758:228, by subsequent designation of Fitzinger, 1843:24.

Diagnosis. Species of Typhlops have (1) eye, distinct, (2) snout, rounded, (3) head scale arrangement, noncircular, (4) frontorostral, absent, (5) nasal, completely divided (rarely incomplete), (6) nasal suture origin, supralabial 2, (7) suboculars or subpreoculars, absent (rarely present), (8) postoculars, 2 (rarely 1 or 3; average, 1.85), (9) preocular-labial contact, supralabial 3 (rarely none), (10) midbody scale rows, 18 or 22 (average, 20.0), (11) scale row reduction, present or absent, (12) total scale rows, 231-457 (average, 312), (13) caudals, 8-19 (average, 13.1), (14) maximum total length, 126-445 (average, 243) mm, (15) total length/midbody diameter, 23-57 (average, 35.9), (16) total length/tail length, 18-88 (36.8), (17) dorsal color, always pigmented, brown or tan, (18) ventral color, unpigmented (pinkish), white, or cream, (19) dorsum darker than venter, and (20) overall, lacking any distinctive pattern (spots, lines, or stripes), although rarely a faint trace of a dorsal line (Tables 1-2); molecular phylogenetic support (Figs. 1, 3).

Among its closest relatives (Figs. 1, 3), Typhlops is distinguished from Cubatyphlops by the presence of 2 postoculars (versus 1; except in 3 species of Typhlops with 1 postocular) and preocular contact with supralabial 3 only (versus contact with supralabials 2 and 3 in Cubatyphlops) (Table 2). The same distinction holds for Typhlops versus the more distantly related Amerotyphlops, although 4 species of that latter genus have more than 1 postocular (Thomas 1968; 1976; Dixon \& Hendricks 1979; Thomas \& Hedges 2007). Typhlops and Antillotyphlops require closest comparison. Thomas (1989) found that species placed here in the genus Typhlops formed a group separate from species placed here in Antillotyphlops based on reduction of the basihyal and a lower number of total middorsal scale rows. He excluded T. jamaicensis and T. sulcatus from that definition, but the molecular data place those two species together with others in Typhlops sensu stricto.

Content. Twenty species: Typhlops agoralionis, T. capitulatus, T. eperopeus, T. gonavensis, T. hectus, T. jamaicensis, T. leptolepis, T. lumbricalis, T. oxyrhinus, T. pachyrhinus, T. proancylops, T. pusillus, T. rostellatus, T. schwartzi, T. silus, T. sulcatus, T. sylleptor, T. syntherus, T. tetrathyreus, and T. titanops (Table 3).

Distribution. Typhlops is distributed primarily on islands in the western Caribbean, including Cuba (and its satellite islets), Hispaniola, and The Bahamas.

Etymology. The generic name, a masculine noun, is a Greek noun meaning 'the blind.'

Remarks. Molecular data are available for nearly all known species in this genus and its monophyly is well -supported. It is also a geographically cohesive group that includes a large number of undescribed species (Fig. 3) (Hedges et al., in preparation).

\section{Discussion}

Although much has been learned about scolecophidians in recent years, mainly through molecular phylogenetic studies, these snakes remain poorly known. There will undoubtedly be many new species discovered in the future, and additional large clades resolved. Scolecophidians are the most ancient group of living snakes and have had a long history on the southern continents (Vidal et al. 2010). The single most common theme that we have encountered in studying the evolution of these snakes is their close association with geography, borne out in this new classification. Their occurrence on islands never connected to continents (Hedges et al. 1992; Hedges 1996a; b; 2008; Vidal et al. 2010) proves that they are capable of dispersing (rafting) over deep water, yet their relationships track plate tectonics perhaps better than any other vertebrate group (Vidal et al. 2010). They have unusually small distributions, with many species known from a single locality, and sympatric species sometimes have no distinguishing scale counts (Hedges \& Thomas 1991; Thomas \& Hedges 2007). This explains why so many undescribed species have been discovered using molecular data (Hedges \& Thomas 1991; Marin et al. 2013b). These features make them an intriguing group to study, for understanding biogeography, ecology, behavior, and speciation. 


\section{Acknowledgments}

This work was funded primarily by a grant from the U.S. National Science Foundation to SBH (DEB-0818798, 'Systematics and biogeography of scolecophidian snakes'), and partly by DEB-1136590 and DEB-0918891. NV and JM were supported by 'Consortium National de Recherche en Génomique' and the 'Service de Systématique Moléculaire' of the Muséum National d'Histoire Naturelle (UMS 2700; OMSI). It is part of agreement number 2005/67 between Genoscope and the Muséum National d'Histoire Naturelle for the project 'Macrophylogeny of life,' directed by Guillaume Lecointre. We thank Lana Brahmakulam, Haley Chutz, Natalie Kardos, and Elisabeth Rochel for laboratory assistance and those persons and institutions who contributed some of the tissue and DNA samples used in these studies or assisted us in the field: Ken Aplin, Aaron Bauer, Christopher Austin, William Branch, L. Chirio, Carla Cicero (MVZ), K. Coate, Ronald Crombie, K. Daoues, Donna Dittmann (LSUMZ), Stephen Donnellan, Paul Doughty, Sandy Echternacht, Julie Feinstein (AMNH, Ambrose-Monnell), Eli Greenbaum, Carla Hass, T. Heger, Robert Henderson, Mark Hutchinson, James Lazell, C. Marty, Greg Mayer, Robert Murphy (ROM), Renata Platenberg, Robert Powell, Nicolas Puillandre, S. Richards, Charles Ross, Richard Thomas, S. Thomson, Sébastien Trape, Miguel Vences, Jens Vindum (CAS), Laurie Vitt, L. Whitsed, and Addison Wynn (USNM). SBH thanks the governments of the Cayman Islands, Cuba, Dominica, Dominican Republic, Guadeloupe, Haiti, Jamaica, Puerto Rico, St. Kitts and Nevis, Turks and Caicos, and the U.S. Virgin Islands for scientific collecting permits. Patrick David provided critical comments on the manuscript.

\section{References}

Adalsteinsson, S.A., Branch, W.R., Trape, S., Vitt, L.J. \& Hedges, S.B. (2009) Molecular phylogeny, classification, and biogeography of snakes of the Family Leptotyphlopidae (Reptilia, Squamata). Zootaxa 2244, 1-50.

Andersson, L.G. (1916) Notes on the reptiles and batrachians in the Zoological Museum at Gothenburg with an account of some new species. Meddelanden fran Göteborgs Musei Zoologiska Afdelning (Göteborg), 17, 1-41.

Aplin, K.P. (1998) Three new blindsnakes (Squamata: Typhlopidae) from northwestern Australia. Records of the Western Australian Museum, 19, 1-12.

Aplin, K.P. \& Donnellan, S.C. (1993) A new species of blindsnake, genus Ramphotyphlops (Typhlopidae, Squamata), from northwestern Western Australia, with a redescription of R. hamatus, Storr 1981. Records of the Western Australian Museum, 16, 243-256.

Auffenberg, W. (1980) The herpetofauna of Komodo, with notes on adjacent areas. Bulletin of the Florida State Museum, Biological Sciences, 25, 39-156.

Barbour, T. (1914) A contribution to the zoögeography of the West Indies, with especial reference to amphibians and reptiles. Memoirs of the Museum of Comparative Zoölogy, 44, 209-359 + 1 plate (includes 7 foldout tables).

Barbour, T. (1921) Reptiles and amphibians from the British Solomon Islands. Proceedings of the New England Zoological Club, 7, 91-112, Pls. 2-6.

Barbour, T. \& Loveridge, A. (1928) A comparative study of the herpetological faunae of the Uluguru and Usambara Mountains, Tanganyika Territory, with descriptions of new species. Memoirs of the Museum of Comparative Zoölogy, 50, 84-265, 4 Pls.

Bauer, A. M. \& Sadlier, R. A. (eds.) (2000). The herpetofauna of New Caledonia. Contributions to Herpetology, 17. Society for Study Amphibians and Reptiles, Ithaca, New York. 322 pp.

Bianconi, J.J. (1847) Specimina zoologica Mosambicana. Memorie della Reale Accademia delle Scienze dell'Istituto di Bologna, Classe di Scienze Fisiche, 1, 171-186.

Bocage, J.V.B.d. (1866) Lista dos reptis das possessões portuguezas d'Africa occidental que existem no Museu de Lisboa. Jornal de Sciencias, Mathematicas, Physicas e Naturaes. Academia Real das Sciencias de Lisboa., 1, 37-56.

Bocage, J.V.B.d. (1873) Reptiles nouveaux de l'intérieur de Mossamedes. Jornal de Sciencias, Mathematicas, Physicas e Naturaes. Academia Real das Sciencias de Lisboa., 4, 247-253, 1 PI.

Bocage, J.V.B.d. (1890) Sur une espèce nouvelle à ajoutter à la faune erpètologique de St. Thomé et Rolas. Jornal 
de Sciencias, Mathematicas, Physicas e Naturaes. Academia Real das Sciencias de Lisboa., (Sér. 2) 2, 61-62.

Böttger, O. (1877) Die Reptilien und Amphibien von Madagascar. Abhandlungen der Senckenbergischen Naturforschenden Gesellschaft, 11, 1-56, 1 PI.

Böttger, O. (1879) Reptilien und Amphibien aus Syrien. Bericht über die Senckenbergische Naturforschende Gesellschaft in Frankfurt am Main, 1878-1879, 57-84.

Böttger, O. (1880) Diagnoses reptilium et batrachiorum novorum a Carolo Ebenau in insula Nossi-Bé Madagascariensi lectorum. Zoologischer Anzeiger, 3, 279-283.

Böttger, O. (1881) Diagnoses Reptilium et Batrachiorum Novorum insulae Nossi-Bé Madagascariensis. Zoologischer Anzeiger, 4, 650-651.

Böttger, O. (1887) Zweiter Beitrag zur Herpetologie Südwest-und Süd-Afrikas. Bericht über die Senckenbergische Naturforschende Gesellschaft in Frankfurt am Main, 1886-1887, 135-173, PI. 5.

Böttger, O. (1897) Neue Reptilien und Batrachier von den Philippinen. Zoologischer Anzeiger, 20, 161-166.

Boulenger, G.A. (1878) Description d'un genre nouveau et d'une expèce nouvelle de la famille des amphisbénides. Bulletin de la Societe Zoologique de France, 3,

Boulenger, G.A. (1887) III. Reptiles., p. 516-517. In: Report on a Zoological Collection made by the Officers of H.M.S. 'Flying-Fish' at Christmas Island, Indian Ocean. Vol. 1887. Günther, A. (ed.)

Boulenger, G.A. (1889) Descriptions of new Typhlopidae in the British Museum. Annals and Magazine of Natural History, (Ser. 6) 4, 360-363.

Boulenger, G.A. (1890) The fauna of British India, including Ceylon and Burma. Reptilia and Batrachia. Taylor and Francis, London, $541 \mathrm{pp}$.

Boulenger, G.A. (1893) Catalogue of the Snakes in the British Museum (Natural History). Volume I, containing the families Typhlopidae, Glauconiidae, Boidae, Ilysiidae, Uropeltidae, Xenopeltidae, and Colubridae Aglyphae, part. Trustees of the British Museum, London, 440 pp.

Boulenger, G.A. (1895a) An account of the reptiles and batrachians collected by Dr. A. Donaldson Smith in western Somaliland and the Galla Country. Proceedings of the Zoological Society of London, 1895, 530-540, 2 Pls.

Boulenger, G.A. (1895b) On a new Typhlops previously confounded with Typhlops unguirostris, Peters. Proceedings of the Linnean Society of New South Wales, (Ser. 2) 9, 718-719.

Boulenger, G.A. (1897) List of the reptiles and batrachians collected by Mr. Alfred Everett in Lombok, Flores, Sumba and Saru, with descriptions of new species. Annals of the Magazine Natural History, (Ser 6.) 19, 503-509.

Boulenger, G.A. (1898a) Descriptions of Two new Blind Snakes. Annals and Magazine of Natural History, (Ser. 7) 1, 124.

Boulenger, G.A. (1898b) Descriptions of Two new Snakes from Queensland. Annals and Magazine of Natural History, (Ser. 7) 2, 414.

Boulenger, G.A. (1900) On a new blind snake from Lifu, Loyalty Islands. In: Willey, A. (Ed.) Zoological Results based on material from New Britain, New Guinea, Loyalty Islands and elsewhere, collected during the years 1895, 1896 and 1897. Cambridge University Press, Cambridge, pp. 603-604.

Boulenger, G.A. (1906) Report on the reptiles collected by the late L. Fea in West Africa. Annali del Museo Civico di Storia Naturale di Genova, (Ser. 3) 2, 196-216.

Boulenger, G.A. (1912) Missione per la frontiera Italo-Etiopica sotto il comando del Capitano Carlo Citerni. Risultati zoologici. List of the reptiles and batrachians. Annali del Museo Civico di Storia Naturale di Genova, (Ser. 3) $5,329-332$.

Bourret, R. (1937) Notes herpétologiques sur l'Indochine française. XV. Lézards et serpents reçu au laboratoire des Sciences Naturelles de I'Université au cours de l'année 1937. Descriptions de deux espèces et de deux variétés nouvelles. Bull. Gen. I'Instr. Publ. Indochine, Hanoi, 57-82

Breuil, M. (1999) Nouvelle espèce du genre Typhlops, (Serpentes, Typhlopidae) de l'île de Saint-Barthélemy, comparaison avec les autres espèces des Petites Antilles. Bulletin Mensuel Société Linnéenne Lyon, 68, 30-40. 
Broadley, D.G. \& Wallach, V. (2000) A new blind snake (Serpentes: Typhlopidae) from montane forests of the Eastern Arc Mountains in Tanzania. African Journal of Herpetology, 49, 165-168.

Broadley, D.G. \& Wallach, V. (2007) A review of East and Central African species of Letheobia Cope, revived from the synonymy of Rhinotyphlops Fitzinger, with descriptions of five new species (Serpentes:Typhlopidae). Zootaxa 1408, 31-68.

Broadley, D.G. \& Wallach, V. (2009) A review of the eastern and southern African blind-snakes (Serpentes: Typhlopidae), excluding Letheobia Cope, with the description of two new genera and a new species. Zootaxa 2255, $1-100$.

Brongersma, L.D. (1934) Contributions to Indo-Australian Herpetology. Zoologische Mededelingen, 17, 161-251, 2 Pls.

Chabanaud, P. (1916) Énumération des Ophidiens non encore étudiés de l'Afrique occidentale, appartenant aux Collections du Muséum, avec la description des espèces et des variétés nouvelles. Bulletin du Museum National d'Histoire Naturelle (Paris), 22, 362-382.

Cogger, H.G. (2000) Reptiles and Amphibians of Australia. Cornell University Press, Ithaca, New York, 775 pp.

Constable, J.D. (1949) Reptiles from the Indian Peninsula in the Museum of Comparative Zoölogy. Bulletin of the Museum of Comparative Zoology, 103, 59-160.

Cope, E.D. (1866) Fourth contribution to the herpetology of tropical America. Proceedings of the Academy of Natural Sciences of Philadelphia, 18, 123-132.

Cope, E.D. (1868a) An examination of the Reptilia and Batrachia obtained by the Orton Expedition to Equador and the upper Amazon, with notes on other species. Proceedings of the Academy of Natural Sciences, Philadelphia, 20, 96-140.

Cope, E.D. (1868b) Observations on Reptiles of the Old World. Art. II. Proceedings of the Academy of Natural Sciences of Philadelphia, 20, 316-323.

Couper, P.J., Covacevich, J.A. \& Wilson, S.K. (1998) Two new species of Ramphotyphlops (Squamata: Typhlopidae) from Queensland. Memoirs of the Queensland Museum, 42, 459-464.

Daudin, F.M. (1803) Histoire naturelle, générale et particulière des reptiles; ouvrage faisant suit à l'histoire naturelle générale et particulière, composée par Leclerc de Buffon; et rédigé par C. S. Sonnini, miembre de plusieurs sociétés savantes. F. Dufart, Paris, 436 pp., Pls. 1-12.

De Jong, J.K. (1930) Notes on some reptiles from the Dutch East Indies. Treubia, 12, 115-119.

De Lang, R. (2011) The snakes of the Lesser Sunda Islands (Nus Tengarra), Indonesia. Asian Herpetological Research, $2,46-54$.

Dixon, J.R. \& Hendricks, F.S. (1979) The wormsnakes (Family Typhlopidae) of the Neotropics, exclusive of the Antilles. Zoologische Verhandelingen, 1-39.

Dominguez, M., Ansel, F.G. \& Iturriaga, M. (2013) A new blind snake (Typhlopidae) from Northeastern Cuba. Zootaxa, 3681, 136-146.

Dominguez, M. \& Diaz Jr., R.E. (2011) Taxonomy of the Blind Snakes Associated with Typhlops lumbricalis (Linnaeus, 1758) (Scolecophidia, Typhlopidae) from the Bahamas Islands and Cuba. Herpetologica, 67, 194-211.

Dominguez, M. \& Moreno, L.V. (2009) Taxonomy of the Cuban blind snakes (Scolecophidia, Typhlopidae), with the description of a new large species. Zootaxa 2028, 59-66.

Doria, G. (1874) Enumerazione dei rettili raccolti dal Dott. O. Beccari in Amboina alle Isole Aru ed alle Isole Kei durante gli anni 1872-1873. Annali del Museo Civico di Storia Naturale di Genova, (Ser. 1) 6, 325-357.

Duméril, A.-M.-C. \& Bibron, G. (1844) Erpétologie générale ou histoire naturelle complète des reptiles. Tome sixième, comprenant l'histoire générale des ophidiens, la description des genres et des espèces de serpents non venimeux, savoir: la totalité des vermiformes ou des scolécophides, et partie des circuriformes ou azémiophides; en tout vingt-cing genres et soixante-cinq espèces. Librairie Encyclopédique de Roret, Paris, i-xii + 1-609 pp.

Duméril, A.-M.-C. \& Duméril, A.-H.-A. (1851) Catalogue méthodique de la collection des reptiles du Muséum d'Histoire 
Naturelle de Paris. Gide et Baudry, Paris, iv + 224 pp.

Duméril, A. (1856) Note sur les Reptiles du Gabon. Revue et Magasin de Zoologie Pure et Appliqueé (Paris), (Sér. 2) 8, 369-377, 417-424, 460-470, 553-562, PI. 21.

Fitzinger, L. (1843) Systema Reptilium. Braumüller et Seidel Bibliopolas, Wien, 106 pp.

FitzSimons, V. (1932) Preliminary descriptions of new forms of South African Reptilia and Amphibia, from the Vernay-Lang Kalahari Expedition, 1930. Annals of the Transvaal Museum, 15, 35-40.

Forcart, L. (1953) Die amphibien und reptilien von Sumba, ihre zoogeographischen beziehungen und revision der unterarten von Typhlops polygrammicus. Verhandlungen der Naturforschenden Gesellschaft in Basel, 64, 356-388.

Franzen, M. \& Wallach, V. (2002) A new Rhinotyphlops from southeastern Turkey (Serpentes : Typhlopidae). Journal of Herpetology, 36, 176-184.

Gans, C. \& Laurent, R.F. (1965) Snakes, p. 47-70, Pls. 6-7. In: Notes on a herpetological collection from the Somali Republic. Vol. 134. Gans, C., Laurent, R.F. \& Pandit, H. (eds.)

Glaw, F. \& Vences, M. (2007) A field guide to the amphibians and reptiles of Madagascar. Vences \& Glaw Verlags GbR, Cologne, Germany, 496 pp.

Grandidier, A. (1872) Descriptions de quelques Reptiles nouveaux découverts á Madagascar en 1870. Annales des Sciences Naturelles. Zoologie et Paléontologie (Paris), (Sér. 5) 15, 6-11.

Gray, J.E. (1845) Catalogue of the specimens of lizards in the collection of the British Museum. Trustees of the British Museum/Edward Newman, London, xxvii + 289 pp.

Guibé, J. (1952) La réduction des membres. In: Grassé, P.-P. (Ed.) Traité de zoologie: anatomie, systématique, biologie. Tome XIV. Reptiles: caractères généraux et anatomie (fascicule II). Masson et Compagnie Éditeurs, Paris, France, pp. 194-201.

Günther, A. (1875) Second report on collections of Indian reptiles obtained by the British Museum. In: Proceedings of the Zoological Society of London. Vol. 224

Günther, A.C.L.G. (1864) The reptiles of British India. Ray Society, London, xxvii + 452 pp.

Hall, T.A. (1999) Bioedit: a user-friendly biological sequence alignment editor and analysis program for Windows 95/98/NT. Nucleic Acids Symposium Series, 41, 95-98.

Hallowell,E. 1848. Description of two new species of Onychocephalus, from the western coast of Africa. Proceedings of the Academy of Natural Sciences, Philadelphia, 1848, 59-61.

Hedges, S.B. (1989) Geographic protein variation in the Jamaican blind snake, Typhlops jamaicensis (Serpentes: Typhlopidae). Caribbean Journal of Science, 25, 93-96.

Hedges, S.B. (1994) Molecular evidence for the origin of birds. Proceedings of the National Academy of Sciences USA, $91,2621-2624$.

Hedges, S.B. (1996a) Historical biogeography of West Indian vertebrates. Annual Review of Ecology and Systematics, 27, 163-196.

Hedges, S.B. (1996b) The origin of West Indian amphibians and reptiles. In: Powell, R. \& Henderson, R.W. (Eds.) Contributions to West Indian Herpetology: A Tribute to Albert Schwartz. Society for the Study of Amphibians and Reptiles, Ithaca, pp. 95-127.

Hedges, S.B. (2008) At the lower limit of size in snakes: two new species of threadsnakes (Squamata: Leptotyphlopidae: Leptotyphlops) from the Lesser Antilles. Zootaxa, 1841, 1-30.

Hedges, S.B. (2011) The type species of the threadsnake genus Tricheilostoma Jan revisited (Squamata, Leptotyphlopidae). Zootaxa 3027, 63-64.

Hedges, S.B., Hass, C.A. \& Maxson, L.R. (1992) Caribbean biogeography: Molecular evidence for dispersal in West Indian terrestrial vertebrates. Proceedings of the National Academy of Sciences of the United States of America, 89, 1909-1913.

Hedges, S.B. \& Thomas, R. (1991) Cryptic species of snakes (Typhlopidae: Typhlops) from the Puerto Rico Bank de- 
tected by protein electrophoresis. Herpetologica, 47, 448-459.

in den Bosch, H.A. \& Ineich, I. (1994) The Typhlopidae of Sulawesi (Indonesia): A Review with Description of a New Genus and a New Species (Serpentes: Typhlopidae). Journal of Herpetology, 28, 206-217.

Ingram, G.J. \& Covacevich, J.A. (1993) Two new species of striped blindsnakes. Memoirs of the Queensland Museum, $34,181-184$.

Jan, G. (1863) Elenco sistematico degli ofidi descritte e disegnati per l'iconografia generale. A. Lombardi, Milan, vii + $143 \mathrm{pp}$.

Jan, G. (1864) Iconographie générale des ophidiens. Atlas. Georges Jan and Ferdinand Sordelli, Paris,pp.

Jiménez, A. \& Savage, J.M. (1963) A New Blind Snake (genus Typhlops) from Costa Rica. Revista de Biologia Tropical, 10, 199-203.

Joger, U. (1990) The herpetofauna of the Central African Republic, with description of a new species of Rhinotyphlops (Serpentes: Typhlopidae). In: Peters, G. \& Hutterer, R. (Eds.) Vertebrates in the tropics. Museum Alexander Koenig, Bonn, pp. 85-102.

Khan, M.S. (1999) Two new species and a subspecies of blind snakes of genus Typhlops from Azad Kashmir and Punjab, Pakistan (Serpentes: Typhlopidae). Russian Journal of Herpetology, 6, 231-240.

Kinghorn, J.R. (1929) Two new snakes from Australia. Records of the Australian Museum, 17, 190-192.

Kinghorn, J.R. (1942) Herpetological Notes No. 4. Records of the Australian Museum, 21, 118-121.

Kornilios, P., Giokas, S., Lymberakis, P. \& Sindaco, R. (2013) Phylogenetic position, origin and biogeography of Palearctic and Socotran blind-snakes (Serpentes: Typhlopidae). Molecular Phylogenetics and Evolution, 68, $35-41$.

Kornilios, P., Ilgaz, C., Kumlutas, Y., Giokas, S., Fraguedakis-Tsolis, S. \& Chondropoulos, B. (2011) The role of Anatolian refugia in herpetofaunal diversity: an mtDNA analysis of Typhlops vermicularis Merrem, 1820 (Squamata, Typhlopidae). Amphibia-Reptilia, 32, 351-363.

Laurent, R.F. (1952) Reptiles et batraciens nouveaux de la région des Grands Lacs africains. Revue de Zoologie et Botanique Africaines, 46, 269-279

Laurent, R.F. (1956) Contribution à I'Herpétologie de la Région des Grands Lacs de l'Afrique centrale. I. Généralités. II. Chéloniens-III. Ophidiens. Annales du Musée Royal du Congo Belge. Série in Octavo, Sciences Zoologiques., 48, 1-390, 31 Pls.

Laurent, R.F. (1964) A revision of the punctatus Group of African Typhlops (Reptilia: Serpentes). Bulletin of the Museum of Comparative Zoology, 130, 387-444.

Leach, W.E. (1819) Appendix. No. IV. Dr. Leach's notice of reptiles, insects, \&c. In: Bowdich, T.E. (Ed.) Mission from Cape Coast castle to Ashantee, with a statistical account of that Kingdom, and geographical notices of other parts of the interior of Africa. John Murray, London, pp. 493-496.

Legler, J.M. (1959) A new blind snake (Genus Typhlops) from Cuba. Herpetologica, 15, 105-112.

Linnaeus, C. (1758) Systema naturæ per regna tria naturæ, secundum classes, ordines, genera, species, cum characteribus, differentiis, synonymis, locis. L. Salvius, Stockholm, 824 pp.

Loveridge, A. (1931) A new snake of the genus Typhlops from the Belgian Congo. Copeia, 1931, 92-93.

Loveridge, A. (1935) Scientific results of an expedition to rain forest regions in Eastern Africa. I. New reptiles and amphibians from East Africa. Bulletin of the Museum of Comparative Zoölogy at Harvard College (Cambridge), 79, 1-19.

Loveridge, A. (1941) Report on the Smithsonian-Firestone Expedition's collection of reptiles and amphibians from Liberia. Proceedings of the United States National Museum, 91, 113-140.

Loveridge, A. (1942) Scientific results of a fourth expedition to forested areas in east and central Africa. Bulletin of the Museum of Comparative Zoology, 91, 237-373.

Loveridge, A. (1945) A new blind snake (Typhlops tovelli) from Darwin, Australia. Proceedings of the Biological Society of Washington, 58, 111-112. 
Marin, J., Donnellan, S.C., Hedges, S.B., Doughty, P., Hutchinson, M.N., Cruaud, C. \& Vidal, N. (2013a) Tracing the history and biogeography of the Australian blindsnake radiation. Journal of Biogeography, 40, 928-937.

Marin, J., Donnellan, S.C., Hedges, S.B., Puillandre, N., Aplin, K.P., Doughty, P., Hutchinson, M.N., Couloux, A. \& Vidal, N. (2013b) Hidden species diversity of Australian burrowing snakes (Ramphotyphlops). Biological Journal of the Linnean Society, 110, 427-441.

McDiarmid, R.W., Campbell, J.A. \& Touré, T.A. (1999) Snake Species of the World. A Taxonomic and Geographic Reference. Herpetologists League, Washington, D.C., $511 \mathrm{pp}$.

McDowell, S.B. (1974) A catalogue of the snakes of New Guinea and the Solomons, with special reference to those in the Bernice P. Bishop Museum. Part I. Scolecophidia. Journal of Herpetology, 8, 1-57.

Merrem, B. (1820) Versuch eines Systems der Amphibien (Tentamen systematis amphibiorum). J. C. Krieger, Marburg, Germany, 191 pp.

Mertens, R. (1927) Neue Amphibien und Reptilien aus dem Indo-Australischen Archipel, gesammelt während der Sunda-Expedition Rensch. Senckenbergiana, 9, 234-242.

Mertens, R. (1929) Bemerkungen über Typhlops florensis. Treubia, Buitenzorg, 11, 293-296.

Mocquard, M.F. (1897) Note sur quelques Reptiles de Tanga, don de M. Gierra. Bulletin du Muséum d'Histoire Naturelle (Paris), 3, 122-123.

Mocquard, M.F. (1901) Note préliminaire sur une collection de Reptiles et de Batraciens recueillis par M. Alluaud dans le sud de Madagascar. Bulletin du Muséum d'Histoire Naturelle (Paris), 7, 251-256.

Montague, P.D., Gonville, B.A. \& Caius College, C.c.b.P.J.S.G. (1914) A Report on the Fauna of the Monte Bello Islands. Proceedings of the Zoological Society of London, 1914, 625-652, 4 Pls.

Niyomwan, P., Thirakhupt, K. \& Nabhitabhata, J. (2001) A Key to the Blind Snakes in Thailand. Natural History, 1, 47-52.

Oppel, M. (1811) Die Ordnungen, Familien und Gattungen der Reptilien, als Prodrom einer Naturgeschichte derselben. Joseph Lindauer, Munich, 86 pp.

Ôshima, M. (1916) A correction of the scientific names of Formosan snakes [In Japanese]. The Zoological Magazine (formerly Dobutsugaku Zasshi...), 28, 84-86.

Palumbi, S.R., Martin, A.P., Romano, S., McMillan, W.O., Stice, L. \& Grabowski, G. (1991) The Simple Fool's Guide to PCR. Version 2.0. Palumbi, Honolulu, Hawaii, pp.

Parker, H.W. (1927) A new Blind Snake from Madagascar. Annals and Magazine of Natural History, (Ser. 9) 19, 379380.

Parker, H.W. (1930) Three new Reptiles from Somaliland. Annals and Magazine of Natural History, (Ser. 10) 6, 603606.

Parker, H.W. (1931) A new Species of Blind Snake from N.W. Australia. Annals and Magazine of Natural History, (Ser. 10) 8, 604-605.

Parker, H.W. (1939) Reptiles and Amphibians from Bougainville, Solomon Islands. Bulletin du Musée Royal d'Histoire Naturelle de Belgique, 15, 1-5.

Peters, W.C.H. (1854) Diagnosen neuer Batrachier, welche zusammen mit der früher (24. Juli und 17. August) gegebenen Übersicht der Schlangen und Eidechen mitgetheilt werden. Bericht über die zur Bekanntmachung geeigneten Verhandlungen der königlich-preussischen Akademie der Wissenschaften zu Berlin, 1854, 614628.

Peters, W.C.H. (1858) Über die Typhlopinen oder blödsichtigen Schlangen und über neue dahin gehörige Arten. Monatsberichte der königlich Akademie der Wissenschaften zu Berlin, 1857, 508-509.

Peters, W.C.H. (1863) Eine Übersicht der von Hrn. Richard Schomburgk an das zoologische Museum eingesandten Amphibien, aus Buchsfelde bei Adelaide in Südaustralien. Monatsberichte der königlich Akademie der Wissenschaften zu Berlin, 1863, 228-236.

Peters, W.C.H. (1864) Über neue Amphibien (Typhloscincus, Typhlops, Asthenodipsas, Ogmodon). Monatsberichte der 
königlich Akademie der Wissenschaften zu Berlin, 1864, 271-276, 1 PI.

Peters, W.C.H. (1865) [Ein fernere] Nachtrag zu [Peters] Abhandlung über Typhlopina. Monatsberichte der königlich Akademie der Wissenschaften zu Berlin, 1865, 259-263, 1 PI.

Peters, W.C.H. (1867a) Herpetologische Notizen. Monatsberichte der königlich Akademie der Wissenschaften zu Berlin, 1867, 13-37.

Peters, W.C.H. (1867b) Über Flederthiere (Pteropus gouldii, Rhinolophus deckenii, Vespertilio lobipes, Vesperugo temminckii) und Amphibien (Hypsilurus godeffroyi, Lygosoma scutatum, Stenostoma narisostre, Onychocephalus unguirostris, Ahaetulla poylepis, Pseudechis scutellatus, Hoplobatrachus reinhardtii, Hyla coriacea). Monatsberichte der königlich Akademie der Wissenschaften zu Berlin, 1867, 703-712, 1 plate.

Peters, W.C.H. (1868) Über eine neue Nagergattung, Chiropodomys penicillatus, so wie über einige neue oder weniger bekannte Amphibien und Fische. Monatsberichte der königlich Akademie der Wissenschaften zu Berlin, 1868, 448-460, 2 Pls.

Peters, W.C.H. (1874) Über einige neue Reptilien (Lacerta, Eremias, Diploglossus, Euprepes, Lygosoma, Sepsina, Ablepharus, Simotes, Onychocephalus). Monatsberichte der königlich Akademie der Wissenschaften zu Berlin, 1874, 368-377, $1 \mathrm{PI}$.

Peters, W.C.H. (1875) Über die von Hrn. Professor Dr. R. Bucholz in Westafrika gesammelten Amphibien. Monatsberichte der königlich Akademie der Wissenschaften zu Berlin, 1875, 196-212, 3 Pls.

Peters, W.C.H. (1877) Herpetologische Notizen. Monatsberichte der königlich preussischen Akademie der Wissenschaften zu Berlin, 1877, 407-423, 1 PI.

Peters, W.C.H. (1878) Über die von Hrn. J. M. Hildebrandt während seiner letzten ostafrikanischen Reise gesammelten Säugethiere und Amphibien. Monatsberichte der königlich preussischen Akademie der Wissenschaften zu Berlin, 1878, 194-209, 2 Pls.

Peters, W.C.H. (1879) Über neue Amphibien des Kgl. zoologischen Museums (Euprepes, Acontias, Typhlops, Zamenis, Spilotes, Oedipus). Monatsberichte der königlich preussischen Akademie der Wissenschaften zu Berlin, 1879, 773-779, 1 PI.

Peters, W.C.H. (1880) Über neue oder weniger bekannte Amphibien des Berliner Zoologischen Museums (Leposoma dispar, Monopeltis (Phractogonus) jugularis, Typhlops depressus, Leptocalamus trilineatus, Xenodon punctatus, Elapomorphus erythronotus, Hylomantis fallax). Monatsberichte der königlich preussischen Akademie der Wissenschaften zu Berlin, 1880, 217-224, 1 Pl.

Peters, W.C.H. (1881) Einige herpetologische Mittheilungen. Sitzungsberichte der Gesellschaft naturforschender Freunde zu Berlin, 1881, 69-72.

Rabosky, D.L., Aplin, K.P., Donnellan, S.C. \& Hedges, S.B. (2004) Molecular phylogeny of blindsnakes (Ramphotyphlops) from Western Australia and Resurrection of Ramphotyphlops bicolor (Peters, 1857). Australian Journal of Zoology, 52, 531-548.

Razzetti, E., Sindaco, R., Grieco, C., Pella, F., Ziliani, U., Pupin, F., Riservato, E., Pellitteri-Rosa, D., Butikofer, L., Suleiman, A.S., Al-Aseily, B.A., Carugati, C., Boncompagni, E. \& Fasola, M. (2011) Annotated checklist and distribution of the Socotran Archipelago Herpetofauna (Reptilia). Zootaxa, 1-44.

Renoult, J.P. \& Raselimanana, A.P. (2009) A new species of Malagasy blind snake of the genus Typhlops Oppel (Serpentes: Typhlopidae). Zootaxa, 65-68.

Richmond, N.D. (1955) The blind snakes (Typhlops) of Bimini, Bahama Islands, British West Indies, with description of a new species. American Museum Novitates, 1-7.

Richmond, N.D. (1964) The blind snakes (Typhlops) of Haiti with descriptions of three new species. Breviora, 1-12.

Richmond, N.D. (1965) A new species of blind snake, Typhlops, from Trinidad. Proceedings of the Biological Society of Washington, 78, 121-124.

Richmond, N.D. (1966) The blind snakes, Typhlops, of Guadeloupe and Dominica with the description of a new species. Herpetologica, 22, 129-132. 
Robb, J. (1966) The generic status of the Australasian typhlopids (Reptilia: Squamata). The Annals and Magazine of Natural History, 675-679.

Robb, J. (1972) A new species of the genus Ramphotyphlops (Serpentes: Typhlopidae) from Western Australia. Journal of the Royal Society of Western Australia, 55, 39-40.

Rodrigues, M.T. (1991) Herpetofauna das dunas interiores do Rio São Francisco, Bahia, Brasil. IV. Uma nova especie de Typhlops (Ophidia, Typhlopidae). Papéis Avulsos de Zoologia, 37, 343-346.

Rodrigues, M.T. \& Juncá, F.A. (2002) Herpetofauna of the quaternary sand dunes of the middle Rio São Francisco, Bahia, Brazil. VII. Typhlops amoipira sp. nov., a possible relative of Typhlops yonenagae (Serpentes, Typhlopidae). Papéis Avulsos de Zoologia, 42, 325-333.

Ronquist, F. \& Huelsenbeck, J.P. (2003) MrBayes 3: Bayesian phylogenetic inference under mixed models. Bioinformatics, 19, 1572-1574.

Rösler, H. \& Wranik, W. (2004) A key and annotated checklist to the reptiles of the Socotra Archipelago. Fauna of Saudi Arabia, 20, 505-534.

Roux-Estève, R. (1974) Révision systématique des Typhlopidae d'Afrique. Reptilia-Serpentes. Mémoires du Muséum National d'Histoire Naturelle (Paris), Série A Zoologie, 87, 1-313, 12 Pls.

Roux-Estève, R. (1980) Une nouvelle espèce de Typhlopidae (Serpentes) du Centre-Est de Madagascar: Typhlops domerguei. Bulletin de Muséum National d'Histoire Naturelle (Paris), (Sér. 4) 2A, 321-323.

Roux, J. (1911) Elbert-Sunda-Expedition des Frankfurter Vereins für Geographie und Statistik. Reptilien und amphibien. Zoologische Jahrbuecher, 30, 495-508.

Roux, J. (1926) Notes d'erpétologie sud-américaine. Revue Suisse de Zoologie, 33, 291-299.

Ruthven, A.G. \& Gaige, H.T. (1935) Observations on Typhlops from Puerto Rico and some of the adjacent islands. Occasional Papers of the Museum of Zoology, University of Michigan., 307, 1-12.

Sackett, J.T. (1940) Preliminary report on results of the West Indies-Guatemala expedition of 1940 for the Academy of Natural Sciences of Philadelphia. Part I.-A new blind snake of the genus Typhlops. Notulae Naturae of the Academy of Natural Sciences of Philadelphia., 48, 1-2.

Salvin, O. (1860) On the Reptiles of Guatemala. Proceedings of the Zoological Society of London, 1860, 451-461.

Savage, J.M. (1950) Two new blind snakes (genus Typhlops) from the Philippine Islands. Proceedings of the California Zoological Club, 1, 49-54.

Savage, J.M. \& Boundy, J. (2012) On the type species of the snake generic name Anilios Gray, 1845 (Serpentes: Typhlopidae). Herpetological Review, 43, 537-538.

Schlegel, H. (1839) Abbildungen neuer oder unvollständig bekannter Amphibien, nach der Natur oder dem Leben entworfen, herausgegeben und mit einem erlänternden Texte begleitet. Arnz \& Comp., Düsseldorf,pp.

Schmidt, K.P. (1923) Contributions to the herpetology of the Belgian Congo based on the collection of the American Museum Congo Expedition, 1909-1915. Part II.-Snakes, with field notes by Herbert Lang and James P. Chapin. Bulletin of the American Museum of Natural History, 49, 1-146, PIs. 1-22.

Schmidt, K.P. (1926) The amphibians and reptiles of Mona Island, West Indies. Field Museum Natural History Zoological Series, 12, 149-163.

Schmidt, K.P. (1936) New amphibians and reptiles from Honduras in the Museum of Comparative Zoology. Proceedings of the Biological Society of Washington, 49, 43-50.

Scortecci, G. (1929a) Primo contributo alla conoscenza dei rettili e degli anfibi della Somalia italiana. Atti della Societa Italiana di Scienze Naturali e del Museo Civico di Storia Naturale di Milano, 68, 245-279, PI. 12.

Scortecci, G. (1929b) Rettili dell'Eritrea esistenti nelle Collezioni del Museo Civico de Milano. Museo civico di storia naturale di Milano. and Societtaliana di scienze naturali, 67, 290-399.

Shaw, G. (1802) General zoology or systematic natural history. Thomas Davison, White-Friars, London, 313-615pp.

Shea, G.M. \& Horner, P. (1997) A new species of Ramphotyphlops (Squamata: Typhlopidae) from the Darwin Area, with notes on two similar species from northern Australia. The Beagle, Records of the Museums and Art 
Galleries of the Northern Territory, 13, 53-60.

Smith, A. (1846) Illustrations of the zoology of South Africa; consisting chiefly of figures and descriptions of the objects of natural history collected during an expedition into the interior of South Africa, in the years 1834, 1835, and 1836; fitted out by "The Cape of Good Hope Association for Exploring Central Africa." Reptilia. Smith, Elder and Co., London, pl. 51, pl. 54, and two unnumbered pages

Stamatakis, A. (2006) RAxML-VI-HPC: maximum likelihood-based phylogenetic analyses with thousands of taxa and mixed models. Bioinformatics, 22, 2688-2690.

Stamatakis, A., Hoover, P. \& Rougemont, J. (2008) A rapid bootstrap algorithm for the RAxML Web servers. Syst Biol, $57,758-71$.

Stejneger, L. (1894) Description of a new species of blind-snakes (Typhlopidae) from the Congo free state. Proceedings of the United States National Museum, [1893] 16, 709-710.

Stejneger, L. (1904) The herpetology of Porto Rico. Annual Reports of the United States National Museum, 1902, 549-724.

Sternfeld, R. (1908) Neue und ungenügend bekannte afrikanische Schlangen. Sitzungsberichte der Gesellschaft naturforschender Freunde zu Berlin, 1908, 92-95.

Sternfeld, R. (1910) Neue Schlangen aus Kamerun, Abessynien u. Deutsch-Ostafrika. Mitteilungen aus dem Zoologischen Museum in Berlin, 5, 67-70.

Sternfeld, R. (1912) Reptilia. In: Schubotz, H. (Ed.) Wissenschaftlich Ergebnisse der deutschen Zentral-Afrika-Expedition 1907-1908 unter Führung Adolf Friedrichs, Herzogs zu Mecklenberg. Band IV. Zoologie II. Lieferung 9. Klinkhardt \& Biermann, Leipzig, pp. 197-279, Pls. 6-9.

Stoliczka, F. (1871) Notes on some Indian and Burmese Ophidians. Journal of the Asiatic Society of Bengal. Part II (Natural History, etc.), 40, 421-445, Pls. 25-26.

Storr, G.M. (1981) The genus Ramphotyphlops (Serpentes: Typhlopidae) in Western Australia. Records of the Western Australian Museum, 9, 235-271.

Storr, G.M. (1983) A new Ramphotyphlops (Serpentes: Typhlopidae) from Western Australia. Records of the Western Australian Museum, 10, 315-317.

Storr, G.M. (1984) A new Ramphotyphlops (Serpentes: Typhlopidae) from central Australia. Records of the Western Australian Museum, 11, 313-314.

Tamura, K., Peterson, D., Peterson, N., Stecher, G., Nei, M. \& Kumar, S. (2011) MEGA5: molecular evolutionary genetics analysis using maximum likelihood, evolutionary distance, and maximum parsimony methods. Molecular Biology and Evolution, 28, 2731-2739.

Tanner, V.M. (1948) Pacific Islands Herpetology No. I. Mariana Islands. A New Species of Typhlops. Great Basin Naturalist, 9, 1-20.

Taylor, E.H. (1917) Snakes and lizards known from Negros, with descriptions of new species and new subspecies. The Philippine Journal of Science. Section D: General Biology, Ethnology, and Anthropology, 12, 353-381, 2 Pls.

Taylor, E.H. (1918) Reptiles of Sulu Archipelago. The Philippine Journal of Science. Section D: General Biology, Ethnology, and Anthropology, 13, 233-267, 3 Pls.

Taylor, E.H. (1919) New or rare Philippine reptiles. Philippine Journal of Science, 14, 105-125.

Taylor, E.H. (1947) Comments on Ceylonese snakes of the genus Typhlops with descriptions of new species. University of Kansas Science Bulletin, 31, 283-298.

Taylor, E.H. (1962) New Oriental reptiles. University of Kansas Science Bulletin, 43, 209-263.

Taylor, E.H. (1965) The serpents of Thailand and adjacent waters. University of Kansas Science Bulletin, 45, 6091096.

Thomas, R. (1965) A new species of Typhlops from the Barahona Peninsula of Hispaniola. Copeia, 1965, 436-439.

Thomas, R. (1966) Leeward Islands Typhlops (Reptilia, Serpentes). Proceedings of the Biological Society of Washing- 
ton, $79,255-266$.

Thomas, R. (1966) A reassessment of the Virigin Islands Typhlops with the description of two new subspecies. Revista de Biologia Tropical, 13, 187-201.

Thomas, R. (1968) The Typhlops biminiensis group of Antillean blindsnakes. Copeia, 1968, 713-722.

Thomas, R. (1974a) A new species of Lesser Antillean Typhlops (Serpentes: Typhlopidae). Occasional Papers of the Museum of Zoology. Louisiana State University., 46, 1-5.

Thomas, R. (1974b) A new species of Typhlops (Serpentes: Typhlopidae) from Hispaniola. Proceedings of the Biological Society of Washington, 87, 11-18.

Thomas, R. (1976) Systematics of Antillean blindsnakes of the genus Typhlops (Serpentes: Typhlopidae). Ph.D. dissertation. Louisiana State University, Baton Rouge, $288 \mathrm{pp}$.

Thomas, R. (1989) The relationships of Antillean Typhlops (Serpentes: Typhlopidae) and the description of three new Hispaniolan species. In: Woods, C.A. (Ed.) Biogeography of the West Indies: Past, Present, and Future. Sandhill Crane Press, Gainesville, Florida, pp. 409-432.

Thomas, R. \& Hedges, S.B. (2007) Eleven new species of snakes of the genus Typhlops (Serpentes: Typhlopidae) from Hispaniola and Cuba. Zootaxa, 1400, 1-26.

Townsend, J.H., Wilson, L.D., Ketzler, L.P. \& Luque-Montes, I.R. (2008) The largest blindsnake in Mesoamerica: a new species of Typhlops (Squamata: Typhlopidae) from an isolated karstic mountain in Honduras. Zootaxa $1932,18-26$.

Uetz, P. \& Hošek, J. (2013) The Reptile Database (http://www.reptile-database.org/). Accessed December 2013

Vanzolini, P.E. (1976) Typhlops brongersmianus, a new name for Typhlops brongersmai Vanzolini, 1972, preoccupied (Serpentes, Typhlopidae). Papéis Avulsos de Zoologia, 29, 247.

Vidal, N., Azvolinsky, A., Cruaud, C. \& Hedges, S.B. (2008) Origin of tropical American burrowing reptiles by transatlantic rafting. Biology Letters, 4, 115-118.

Vidal, N., Marin, J., Morini, M., Donnellan, S., Branch, W.R., Thomas, R., Vences, M., Wynn, A., Cruaud, C. \& Hedges, S.B. (2010) Blindsnake evolutionary tree reveals long history on Gondwana. Biology Letters, 6, 558-561.

Waite, E.R. (1893) Notes on Australian Typhlopidae. Records of the Australian Museum, 2, 57-62.

Waite, E.R. (1894) Notes on Australian Typhlopidae. Proceedings of the Linnean Society of New South Wales, (Ser. 2) 9, 9-14, 1 PI.

Waite, E.R. (1897a) A new Blind Snake from the Duke of York Island. Records of the Australian Museum, 3, 69-70.

Waite, E.R. (1897b) Notes on Australian Typhlopidae. Transactions of the Royal Society of South Australia, 21, 25-27, PI. 3.

Waite, E.R. (1918a) Description of a new blind snake from the Solomon Islands, with a list of species from the Austro-Malayan and Polynesian sub-regions. Records of the South Australian Museum, 1, 35-38.

Waite, E.R. (1918b) Review of the Australian blind snakes (Family Typhlopidae). Records of the South Australian Museum, 1, 1-34, $1 \mathrm{PI}$.

Wall, F. (1908) Notes on a collection of snakes from Persia. Journal of the Bombay Natural History Society, 18, 795805.

Wall, F. (1919) Notes on a collection of Snakes made in the Nilgiri Hills and the adjacent Wynaad. Journal of the Bombay Natural History Society, 26, 552-584, 3 Pls.

Wallach, V. (1993) A new species of blind snake, Typhlops marxi, from the Philippines (Serpentes, Typhlopidae). Raffles Bulletin of Zoology, 41, 263-278.

Wallach, V. (1995) A new genus for the Ramphotyphlops subocularis Species Group (Serpentes: Typhlopidae), with description of a new species. Asiatic Herpetological Research, 6, 122-150.

Wallach, V. (1999) Typhlops meszoelyi, a new species of blind snake from northeastern India (Serpentes : Typhlopidae). Herpetologica, 55, 185-191.

Wallach, V. (2000) Critical review of some recent descriptions of Pakistani Typhlops by M. S. Khan, 1999 (Serpentes: 
Typhlopidae). Hamadryad, 25, 129-143.

Wallach, V. (2001) Typhlops roxaneae, a new species of Thai blindsnake from the $T$. diardii species group, with a synopsis of the Typhlopidae of Thailand (Serpentes: Scolecophidia). Raffles Bulletin of Zoology, 49, 39-49.

Wallach, V. (2002) Typhlops etheridgei, a new species of African blindsnakes in the Typhlops vermicularis species group from Mauritania. Hamadryad, 27, 108-122.

Wallach, V. (2003) Scolecophidia miscellanea. Hamadryad, 27, 222-240.

Wallach, V. (2005) Letheobia pauwelsi, a new species of blindsnake from Gabon (Serpentes : Typhlopidae). African Journal of Herpetology, 54, 85-91.

Wallach, V. (2006) The nomenclatural status of Australian Ramphotyphlops (Serpentes: Typhlopidae). Bulletin of the Maryland Herpetological Society, 42, 8-24.

Wallach, V., Brown, R.M., Diesmos, A.C. \& Gee, G.V.A. (2007a) An enigmatic new species of blind snake from Luzon Island, Northern Philippines, with a synopsis of the Genus Acutotyphlops (Serpentes: Typhlopidae). Journal of Herpetology, 41, 690-702.

Wallach, V. \& Glaw, F. (2009) A new mid-altitude rainforest species of Typhlops (Serpentes: Typhlopidae) from Madagascar with notes on the taxonomic status of T. boettgeri Boulenger, T. microcephalus Werner, and T. capensis Rendahl. Zootaxa 2294, 23-38.

Wallach, V., Mercurio, V. \& Andreone, F. (2007b) Rediscovery of the enigmatic blind snake genus Xenotyphlops in northern Madagascar, with description of a new species (Serpentes: Typhlopidae). Zootaxa 1402, 59-68.

Wallach, V. \& Pauwels, O.S.G. (2004) Typhlops lazelli, a new species of Chinese blindsnake from Hong Kong (Serpentes: Typhlopidae). Breviora, Museum of Comparative Zoology, 512, 1-21.

Wallach, V. \& Pauwels, O.S.G. (2008) The systematic status of Cathetorhinus melanocephalus Duméril \& Bibron, 1844 (Serpentes: Typhlopidae). Hamadryad, 32, 116-124.

Wegener, J.E., Swoboda, S., Hawlitschek, O., Franzen, M., Wallach, V., Vences, M., Nagy, T., Hedges, S.B., Köhler, J. \& Glaw, F. (2013) Morphological variation and taxonomic reassessment of the endemic Malagasy blind snake family Xenotyphlopidae (Serpentes, Scolecophidia). Spixiana, 36, 269-282.

Werner, F. (1901) Ueber Reptilien und Batrachier aus Ecuador und Neu-Guinea. Verhandlungen der Zoologisch-Botanischen Gesellschaft in Wien, 51, 593-614, PI. 5.

Werner, F. (1909a) Beschreibung neuer Reptilien aus dem Kgl. Naturalienkabinett in Stuttgart. Jahreshefte des Vereins für vaterländische Naturkunde Württemberg, 65, 55-63.

Werner, F. (1909b) Über neue oder seltene Reptilien des Naturhistorischen Museums in Hamburg. I. Schlangen. Mitteilungen aus dem Natuhistorischen Museum in Hamburg, 26 (1908), 205-247.

Werner, F. (1917) Über einige neue Reptilien und einen neuen Frosch des Zoologischen Museums in Hamburg. Mitteilungen aus dem Naturhistorischen Museum in Hamburg, 34, 31- 36.

Winnepenninckx, B., Backeljau, T. \& Dewachter, R. (1993) Extraction of high molecular weight DNA from molluscs. Trends in Genetics, 9, 407.

Witte, G.-F.d. (1953) Reptiles. In: Exploration du Parc National de l'Upemba. Mission G. F. de Witte en collaboration avec W. Adam, A. Janssens, L. Van Meel et R. Verheyen (1946-1949). Institut des Parcs Nationaux du Congo Belge, Bruxelles, pp. 1-322, Pls. 1-41.

Wynn, A., Reynolds, R.P., Buden, D.W., Falanruw, M. \& Lynch, B. (2012) The unexpected discovery of blind snakes (Serpentes: Typhlopidae) in Micronesia: two new species of Ramphotyphlops from the Caroline Islands. Zootaxa, 3172, 39-54.

Wynn, A.H. \& Leviton, A.E. (1993) Two new species of blind snake, genus Typhlops (Reptilia: Squamata: Typhlopidae), from the Philippine Archipelago. Proceedings of the Biological Society of Washington, 106, 34-35. 


\section{Appendix 1: samples used in the molecular analyses}

The following is a list of samples and localities used in the molecular analyses (Figs. 1-3), excluding those already detailed in our previous global study (Vidal et al. 2010). Besides the samples listed below, we include in Fig. 1 sequences, from GenBank, of Xerotyphlops vermicularis (" $\mathrm{A}$ "), two samples of $X$. socotranus, two of Letheobia episcopa, and one of $L$. simonii published in a recent study (Kornilios et al. 2013). Abbreviations are: CAS (California Academy of Sciences, USA), EBG and ELI (Eli B. Greenbaum), SBH (S. Blair Hedges, Pennsylvania State University, USA), and USNM (National Museum of Natural History, Washington, D.C., USA).

Afrotyphlopinae. Afrotyphlops elegans 1 (CAS 219176; Fonta de Tchipique, Principe Island, Sao Tome and Principe), Afrotyphlops elegans 2 (CAS 238870; Principe Island, Sao Tome and Principe), Afrotyphlops elegans 3 (CAS 238880; abandoned Rosa, Nova Cuba, Principe Island, Sao Tome and Principe), Afrotyphlops sp. 1 (USNM 576137; Impongui, Likouala Dept., Republic of Congo), Afrotyphlops sp. 2 (ELI 158; Katanga Prov., Kasongomwana, Democratic Republic of the Congo), Afrotyphlops cf angolensis (EBG 2220; Force Bendera, on west side of Mt. Kabobo, 30$40 \mathrm{~km}$ N Kalemie, Democratic Republic of the Congo), Letheobia feae 1 (CAS 218907; on road between Bombaim and Santa Adelaide at Rio Abade bridge, Sao Tome Island, Sao Tome and Principe), Letheobia feae 2 (CAS 219310; Cruzeiro, Sao Tome Island, Sao Tome and Principe), Letheobia feae 3 (CAS 219335; trail between Born Sucesso to Lago Ameilia, Sao Tome Island, Sao Tome and Principe).

Asiatyphlopinae. Acutotyphlops subocularis (ABTC104792; Vuovo Camp, West New Britain, PNG). All Anilios are from Australia: Anilios affinis SH1 (SAMAR55645; Blackdown Tableland Road, 2k S Jnctn, QLD), Anilios ammodytes SH10 (WAMR127760; 5k S Mount Tom Price Mine, WA), Anilios ammodytes SH11 (WAMR104109; 1.4k NNE Woodstock, WA), Anilios ammodytes SH2 (WAMR170658; 31k WSW Mt Elvire, WA), Anilios ammodytes SH3 (WAMR121995; Weeli Wolli Spring, WA), Anilios ammodytes SH4 (WAMR102560; Barlee Range Nature Reserve, WA), Anilios ammodytes SH5 (WAMR165282; 16.6k ENE Karratha, WA), Anilios ammodytes SH6 (WAMR139430; Mount Minnie Homestead, WA), Anilios ammodytes SH7 (WAMR162120; 16k W Mt De Courcey, WA), Anilios ammodytes SH8 (WAMR141306; Cape Preston area, WA), Anilios ammodytes SH9 (WAMR158097; 5k NNE Python Pool, WA), Anilios australis SH12 (WAMR132329; 2k NE Wonnerup Siding, WA), Anilios australis SH13 (WAMR154969; East Wallabi Island, WA), Anilios bicolor SH14 (SAMAR61971; near Boolcoomata HS, SA), Anilios bicolor SH15 (WAMR165618; Ora Banda, WA), Anilios bituberculatus SH16 (SAMAR48359; 3.5k W Inkerman, SA), Anilios bituberculatus SH17 (SAMAR62428; 20k NE Mount Penrhyn, SA), Anilios bituberculatus SH18 (SAMAR44731; Welbourne Hill Station, SA), Anilios bituberculatus SH19 (ANWCR06665; Berrigan State Forest, 11k E Berrigan, NSW), Anilios bituberculatus SH20 (SAMAR18867; Burke's Grave, near Innamincka, SA), Anilios centralis SH21 (SAMAR56511; 8k ENE Mount Chandler, SA), Anilios centralis SH22 (ABTC24079; MacDonnell Ranges, NT), Anilios diversus SH23 (WAMR157402; Tanami Desert, WA), Anilios diversus SH24 (NTMR16427; Supplejack Station, 8 Mile Bore, NT), Anilios diversus SH25 (WAMR126054; 5k S Carlton Hill Homestead, WA), Anilios diversus SH26 (WAMR151035; Lake Argyle, WA), Anilios diversus SH27 (WAMR119543; Mirima National Park, WA), Anilios diversus SH28 (ABTC102746; 176 Camooweal Street, Mount Isa, QId), Anilios diversus SH29 (ABTC70684; Tennant Creek, NT), Anilios diversus SH30 (WAMR112027; Beagle Bay Aboriginal Community, WA), Anilios diversus SH31 (NTMR19058; Guluwuru Island, NT), Anilios endoterus SH32 (SAMAR21202; Olympic Dam Site, SA), Anilios endoterus SH33 (WAMR102627; Little Sandy Desert, WA), Anilios endoterus SH34 (WAMR135151; near Telfer Dome, WA), Anilios endoterus SH35 (WAMR115000; 38k ENE Laverton, WA), Anilios ganei SH36 (WAMR140003; Millstream, WA), Anilios ganei SH37 (WAMR162129; 27k ESE Mt De Courcey, WA), Anilios ganei SH38 (WAMR124835; 30k W Newman, Cathederal Gorge, WA), Anilios ganei SH39 (WAMR156328; Chichester Range, WA), Anilios grypus SH40 (WAMR114909; Marble Bar, WA), Anilios grypus SH41 (WAMR108596; 8k E Yarra Bluff, WA), Anilios grypus SH42 (WAMR157403; Tanami Desert, WA), Anilios grypus SH43 (WAMR108923; 25k SE Telfer, WA), Anilios grypus SH44 (WAMR110716; Brockan Mine, WA), Anilios grypus SH45 (WAMR114282; Wittenoom, WA), Anilios grypus SH46 (WAMR127522; 10k S Onslow, WA), Anilios grypus SH47 (WAMR102679; Little Sandy Desert, WA), Anilios grypus SH48 (SAMAR55272; Phosphate Hill, Buffel site, Qld), Anilios guentheri SH49 (WAMR105974; Kingston Rest, WA), Anilios guentheri SH50 (WAMR108431; 32k N Ord River crossing, WA), Anilios 
guentheri SH51 (NTMR16488; Top Springs, Tanami Desert, NT), Anilios guentheri SH52 (NTMR13611; Katherine Gorge, NT), Anilios guentheri SH53 (ABTC67980; Bradshaw Station, NT), Anilios guentheri SH54 (SAMAR53885; 40k S Tunnel Creek Gorge, WA), Anilios hamatus SH55 (WAMR136817; Lake Mason Station, WA), Anilios hamatus SH56 (WAMR156249; Onslow area, WA), Anilios hamatus SH57 (WAMR145266; 5k S Mount Tom Price Mine, WA), Anilios hamatus SH58 (WAMR120619; unknown locality, WA), Anilios hamatus SH59 (WAMR111862; 26k SE Wheelarra Hill, WA), Anilios hamatus SH60 (WAMR136276; Muggon Station, WA), Anilios hamatus SH61 (WAMR131753; Mount Robinson, West Angelas, WA), Anilios howi SH62 (WAMR141536; Kalumburu, WA), Anilios kimberleyensis SH63 (WAMR165559; Koolan Island, WA), Anilios kimberleyensis SH64 (ABTC29484; Litchfield NP, NT), Anilios kimberleyensis SH65 (WAMR165885; South Maret Island, WA), Anilios kimberleyensis SH66 (WAMR125981; Wyndham, WA), Anilios leptosoma SH67 (WAMR114894; Geraldton, WA), Anilios leptosoma SH68 (WAMR129778; 22k S Kalbarri, WA), Anilios ligatus SH69 (SAMR31019; 21k E Narrabri, NSW), Anilios ligatus SH70 (NTMR13753; Keep River NP, NT), Anilios ligatus SH71 (NTMR35156; Tableland Highway, NT), Anilios ligatus SH72 (NTMR19109; Raragala Island, NT), Anilios longissimus SH73 (WAMR120049; Bandicoot Bay, Barrow Island, WA), Anilios nigrescens SH74 (AMSR146228; Castlereagh waste depot, near Penrith, NSW), Anilios nigrescens SH75 (SAMAR31025; 8k WWoolbrook, NSW), Anilios nigrescens SH76 (AMSR157200; Torrington State Recreation area, Butler, NSW), Anilios nigrescens SH77 (ABTC03939; 8k W McAfees Lookout, Qld), Anilios nigrescens SH78 (AMSR157267; Red Range to Kingsgate Road, NSW), Anilios pilbarensis SH79 (WAMR1 10939; 22.1k WSW Pannawonica, WA), Anilios pinguis SH80 (WAMR146995; Helena Valley, WA), Anilios proximus SH83 (ANWCR06668; Sloane Reserve, 28.5k N Mulwala, Savernake Hall, NSW), Anilios silvia SH84 (QMJ46128; 25k N Pomona, Qld), Anilios torresianus SH82 (QMJ60625; Mt Hartley, Qld), Anilios troglodytes SH85 (WAMR146048; Oobagooma Homestead, WA), Anilios unguirostris SH86 (WAMR146958; Kalumburu, WA), Anilios unguirostris SH87 (NTMR21669; Dundee Beach, Fog Bay, NT), Anilios unguirostris SH88 (SAMR54430; Burke Development Road 10k ENE, Qld), Anilios unguirostris SH89 (WAMR117861; near Mount Percy, WA), Anilios waitii SH90 (WAMR120315; unknown locality, WA), Anilios waitii SH91 (WAMR165022; 1k N Mulga Downs Outcamp, WA), Anilios wiedii SH92 (AMSR154152; Poison Gate road, on road to Poison, NSW). Asiatyphlops diardii (USNM, uncataloged; Myanmar), Asiatyphlops muelleri (CAS 222410; Nat Ma Taung National Park, Min Dat Township, Min Dat District, Chin State, Myanmar), Asiatyphlops sp. 1 (CAS 224653; Nagmung Town, Nagmung Township, Putao District, Kachin State, Myanmar), Asiatyphlops sp. 2 (CAS 224658; Nagmung Town, Nagmung Township, Putao District, Kachin State, Myanmar), Asiatyphlops sp. 3 (CAS 224750; between Nagmung Town and Ta Se Htu Village, Nagmung Township, Putao District, Kachin State, Myanmar), Asiatyphlops sp. 4 (CAS 225173; Ba Bawt village, Nagmung Township, Putao District, Kachin State, Myanmar), Asiatyphlops sp. 5 (CAS 230225; Machanbaw Town, Machanbaw Township, Putao District, Kachin State, Myanmar), Asiatyphlops sp. 6 (CAS 235322; near Myin Che Taung, Mintatt Township, Mindat District, Chin State, Myanmar), Asiatyphlops sp. 7 (CAS 235378; between Sawn Taung village and Ba Late Htwe village, Mintatt Township, Mindat District, Chin State, Myanmar), Sundatyphlops polygrammicus (WAM R98715; Brang Kua, Moyo Island, Indonesia), Xerotyphlops vermicularis 1 (CAS 228715; Harzevil Village in Old Manjil, Gilan Province, Iran), Xerotyphlops vermicularis 2 (CAS 228716; Harzevil Village in Old Manjil, Gilan Province, Iran).

Typhlopinae. Antillotyphlops geotomus (SBH 172760; ca. 1.5 km NE New River, Nevis, St. Kitts and Nevis), Antillotyphlops granti (SBH 172155; 4.0 km E Guanica in Bosque Estatal de Guanica, Campamento Santiago, Puerto Rico, USA), Antillotyphlops guadeloupensis (SBH 102276; Pointe de la Grande Anse near Trois Rivieres, Basse-Terre, Guadeloupe), Typhlops hectus (SBH 191789; 2.4 km N of Ducis, Dept. Du Sud, Haiti), Antillotyphlops hypomethes (SBH 172150; Rio Piedras on campus of UPR, Puerto Rico, USA), Typhlops jamaicensis (SBH 172445; $6.2 \mathrm{~km} \mathrm{~W}$ Oracabessa, St. Mary, Jamaica), Antillotyphlops naugus (SBH 172758; Upper Quail Dove Ghut, Guana Island, British Virgin Islands), Cubatyphlops perimychus (SBH 161981; W. T. Sampson High School, Guantanamo Bay USNS, Cuba), Antillotyphlops platycephalus (SBH 172180; 12.3 km SSE Arecibo, Puerto Rico, USA), Typhlops proancylops (SBH 103605; N of Fond Verrettes, Soliette, I'Ouest, Haiti), Typhlops pusillus (SBH 192420; 19.5 km N of Ca Soleil, Dept. de l'Artibonite, Haiti), Antillotyphlops richardii (SBH 266855; 0.2 km S St. Peter's Peak along roadside, St. Thomas, 
US Virgin Islands), Amerotyphlops tasymicris (SBH 269088; Grenadines, Union Island, Chatham Bay), Typhlops titanops (SBH 160293; 22 km N Pedernales at the Rio Mulito, Pedernales, Dominican Republic), Typhlops sp. 01 (SBH 190612; 1.5 km WSW La Tabla, Santiago de Cuba, Cuba), Typhlops sp. 02 (SBH 190943; ca 0.5 km N La Hembrita, Guantanamo, Cuba), Typhlops sp. 03 (SBH 193810; grounds of Hotel Punta de Piedra, Granma, Cuba), Typhlops sp. 04 (SBH 194029; 8.5 km SW of Loma Mensura, Holguin, Cuba), Typhlops sp. 05 (SBH 193770; 2.4 km SE of Ojo del Toro, Granma, Cuba), Typhlops sp. 06 (SBH 193733; 1.6 km N of Camaron Grande, Granma, Cuba), Typhlops sp. 07 (SBH 193614; 1.4 km N of Minas del Frio, Granma, Cuba), Typhlops sp. 08 (SBH 191419; 1.5 km WSW La Tabla, Santiago de Cuba, Cuba), Typhlops sp. 09 (SBH 191538; Narigon, La Habana, Cuba), Typhlops sp. 11 (SBH 190202; SW slope of El Yunque de Baracoa, Guantanamo, Cuba), Typhlops sp. 12 (SBH 190231; 15.8 km NW Baracoa on coast road, Guantanamo, Cuba), Typhlops sp. 13 (SBH 190565; 1.5 km NE La Cantera, Santiago de Cuba, Cuba), Typhlops sp. 14 (SBH 190941; Rio Cuzco Canyon, ca. 3 km N Los Oasis, Guantanamo, Cuba), Typhlops sp. 15 (SBH 191018; near La Fangosa, 10.1 km W Vega Grande, Guantanamo, Cuba), Typhlops sp. 16 (SBH 102370; ca. 15 km W El Valle, Hato Mayor, Dominican Republic), Typhlops sp. 17 (SBH 193206; 8.8 km N thence $0.5 \mathrm{~km}$ W Tenares, Los Tabucos, Dominican Republic), Typhlops sp. 18 (SBH 267752; ca. 1 km SW Los Limones, Monte Plata, Dominican Republic), Typhlops sp. 19 (SBH 266322; 1.6 mi. NNE El Azul, San Juan, Dominican Republic), Typhlops sp. 20 (SBH 191839; 5.0 km S of Pestel, Dept. de la Grand'Anse, Haiti).

Leptotyphlopidae. Leptotyphlops cf emini (EBG 2280; Goma, Democratic Republic of the Congo). 